Florida International University

FIU Digital Commons

7-8-2020

\title{
Structural Health Monitoring of Pipelines in Radioactive Environments Through Acoustic Sensing and Machine Learning
}

Michael Thompson

mthom158@fiu.edu

Follow this and additional works at: https://digitalcommons.fiu.edu/etd

Part of the Acoustics, Dynamics, and Controls Commons, Electromagnetics and Photonics Commons, Industrial Technology Commons, Signal Processing Commons, and the Systems Engineering Commons

\section{Recommended Citation}

Thompson, Michael, "Structural Health Monitoring of Pipelines in Radioactive Environments Through Acoustic Sensing and Machine Learning" (2020). FIU Electronic Theses and Dissertations. 4458. https://digitalcommons.fiu.edu/etd/4458

This work is brought to you for free and open access by the University Graduate School at FIU Digital Commons. It has been accepted for inclusion in FIU Electronic Theses and Dissertations by an authorized administrator of FIU Digital Commons. For more information, please contact dcc@fiu.edu. 


\section{FLORIDA INTERNATIONAL UNIVERSITY \\ Miami, Florida}

STRUCTURAL HEALTH MONITORING OF PIPELINES IN RADIOACTIVE ENVIRONMENTS THROUGH ACOUSTIC SENSING AND MACHINE LEARNING

A thesis submitted in partial fulfillment of the

requirements for the degree of

MASTER OF SCIENCE

in

ELECTRICAL ENGINEERING

by

Michael W. Thompson

2020 
To: Dean John L. Volakis

College of Engineering and Computing

This thesis, written by Michael W. Thompson, and entitled Structural Health Monitoring of Pipelines in Radioactive Environments Through Acoustic Sensing and Machine Learning, having been approved in respect to style and intellectual content, is referred to you for judgment.

We have read this thesis and recommend that it be approved.

$\begin{array}{r}\hline \text { Leonel Lagos } \\ \hline \text { Aparna Aravelli } \\ \hline \text { Ou Bai, Major Professor Bhansali }\end{array}$

Date of Defense: July 8, 2020

This thesis of Michael W. Thompson has been approved.

Dean John L. Volakis College of Engineering and Computing

Andrés G. Gil

Vice President for Research and Economic Development and Dean of the University Graduate School

Florida International University, 2020 


\section{ACKNOWLEDGMENTS}

First and foremost, I would like to thank my advisor Dr. Aparna Aravelli for mentoring me for the last year and a half on my journey through graduate school. She has shown me patience that I do not deserve and shown me the ropes in academics that I otherwise would not have found. For this, I am profoundly grateful.

I would like to thank my committee chair Dr. Ou Bai for giving me valuable insights into my thesis and guiding me through the process.

My life would not be what it was today if not for Dr. Bhansali and the rest of the faculty of the FIU Electrical Engineering department who have given me more opportunities than I can count to pursue research. Dr. Mubarak Mujawar provided me more guidance and support during my college career than I could have hoped for and I am indebted to his kindness.

Next, I would like to personally thank Dr. Leonel Lagos and everyone at the FIU Applied Research Center and DOE Fellows program. Without the work that Dr. Lagos has done to run such a great program I would not have gained half the valuable experience from graduate school that I have today, nor would have I challenged myself as much as I have.

Finally, I must express my deepest gratitude to my family and friends for their support during this long journey. And to the many more students and faculty I have met along the way at FIU that without, none of this would have been possible.

The research presented is a part of the cooperative agreement between FIU and the DOE (\#DE-EM0000598) and a special thanks to everyone at the DOE and National Labs. 


\begin{abstract}
OF THE THESIS
STRUCTURAL HEALTH MONITORING OF PIPELINES IN RADIOACTIVE ENVIRONMENTS THROUGH ACOUSTIC SENSING AND MACHINE LEARNING

by
\end{abstract}

Michael W. Thompson

Florida International University, 2020

Miami, Florida

Professor Ou Bai, Major Professor

Structural health monitoring (SHM) comprises multiple methodologies for the detection and characterization of stress, damage, and aberrations in engineering structures and equipment. Although, standard commercial engineering operations may freely adopt new technology into everyday operations, the nuclear industry is slowed down by tight governmental regulations and extremely harsh environments. This work aims to investigate and evaluate different sensor systems for real-time structural health monitoring of piping systems and develop a novel machine learning model to detect anomalies from the sensor data. The novelty of the current work lies in the development of an LSTM-autoencoder neural network to automate anomaly detection on pipelines based on a fiber optic acoustic transducer sensor system. Results show that pipeline events and faults can be detected by the MLM developed, with a high degree of accuracy and low rate of false positives even in a noisy environment near pumps and machinery. 


\section{TABLE OF CONTENTS}

CHAPTER

PAGE

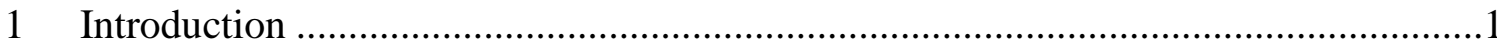

$1.1 \quad$ Structural Health Monitoring ………………………................................... 1

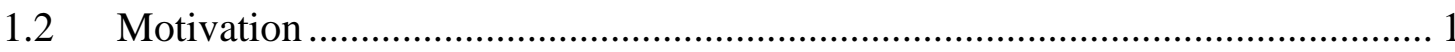

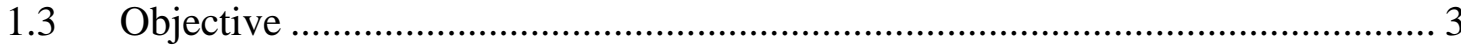

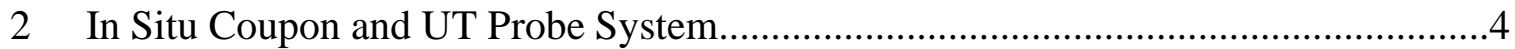

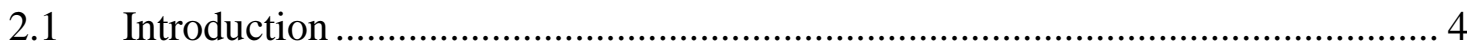

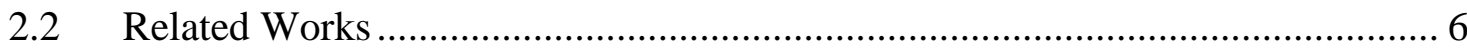

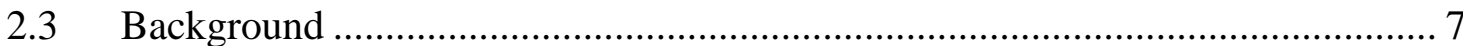

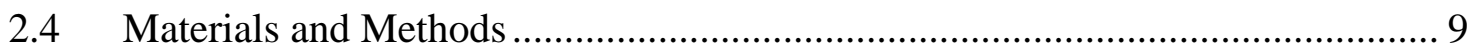

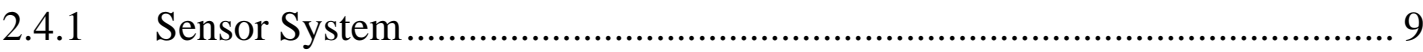

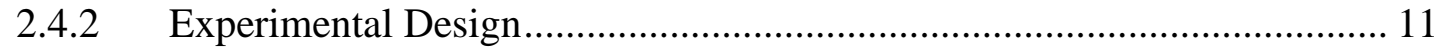

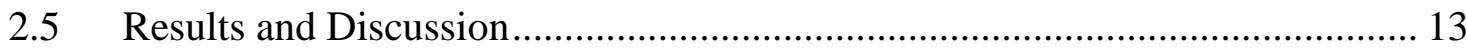

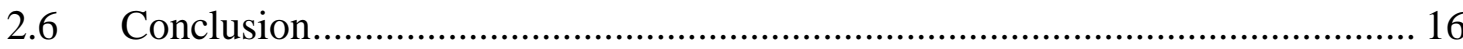

3 Fiber Optic Acoustic Transducers ………………..........................................18

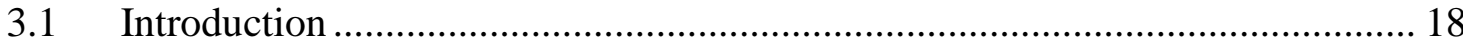

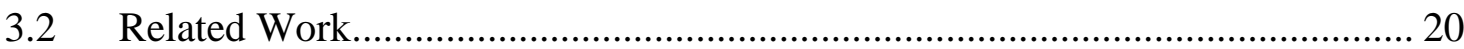

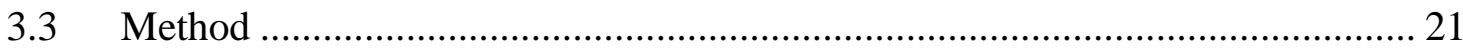

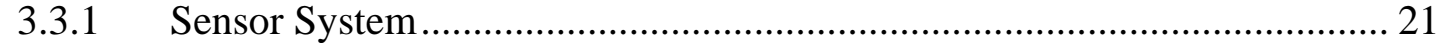

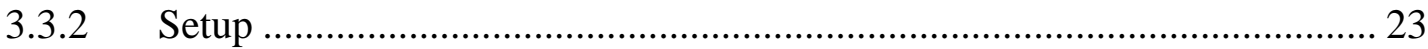

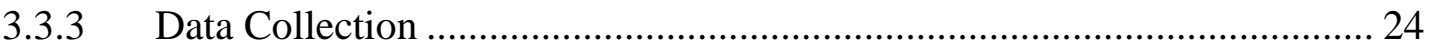

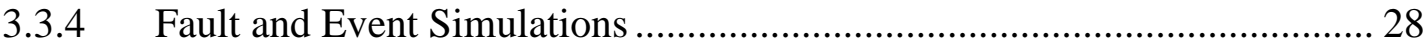

3.3.5 Feature Extraction Algorithms........................................................... 29

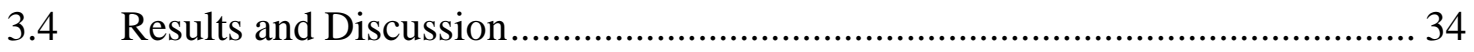

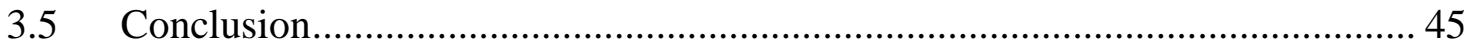

4 Machine Learning Model for Pipeline Fault Detection...............................................46

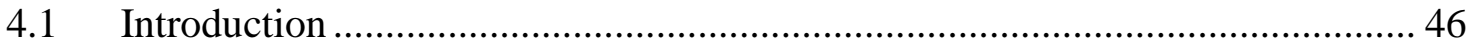




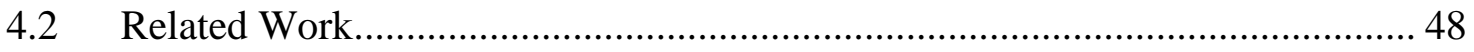

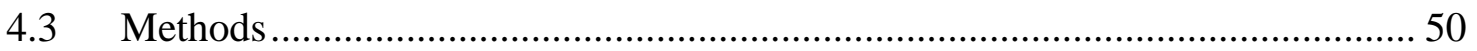

4.3.1 General Architecture ...................................................................... 50

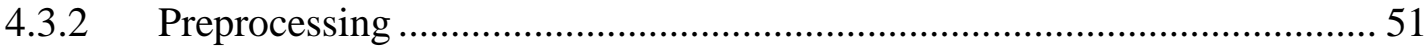

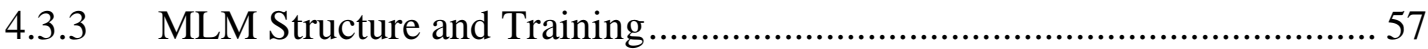

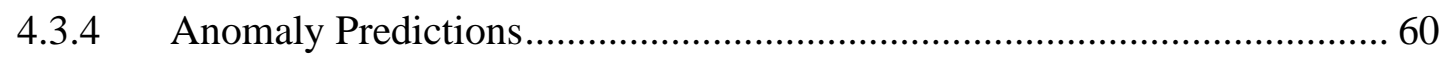

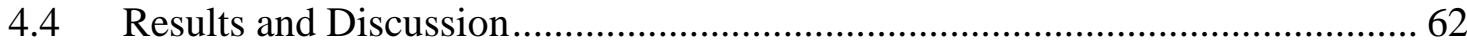

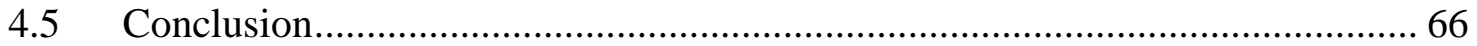

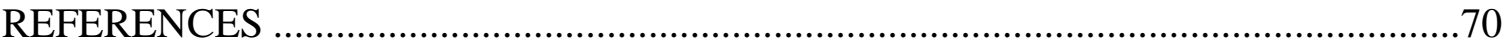




\section{LIST OF TABLES}

TABLE

PAGE

Table 1 - SRNL Coupon Mass and Height Loss......................................................... 16 


\section{LIST OF FIGURES}

FIGURE

PAGE

Figure 2.1 - Ultrasonic Thickness Gauge Diagram ................................................... 7

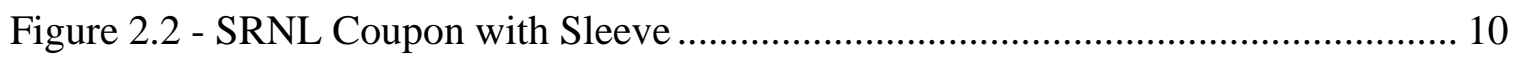

Figure 2.3 - Coupon Inserted into coupling affixed to pipeline .................................. 10

Figure 2.4 - Modified Olympus V260-SM UT Pencil Probe......................................... 10

Figure 2.5 - Bench Scale Pipe Loop Setup ......................................................... 12

Figure 2.6 - Collecting UT probe reading from installed coupon................................. 13

Figure 2.7 - SRNL Coupons’ Eroded Surfaces ..................................................... 14

Figure 2.8 - Erosion Patterns of Coupon Surfaces Under 5X Magnification .................. 15

Figure 3.1 - CEL Fiberstrike Pipeline Monitoring System Architecture [55] ................. 22

Figure 3.2 - CEL Fiberstrike Acoustic Transducer without protective cover [55] .......... 22

Figure 3.3 - CEL Fiberstrike Acoustic Transducer Installed on Pipe ............................. 23

Figure 3.4 - LCM-2500 Interrogator and User Control Station................................... 24

Figure 3.5 - CEL Fiberstrike Data Aquisition Software .......................................... 25

Figure 3.6 - Raw UDP Packet Data From LCM-2500............................................. 27

Figure 3.7 - Sensor Value Conversion from UDP Packet Format ................................ 27

Figure 3.8- Leaks Under Normal Operation ...................................................... 28

Figure 3.9 - Fourier Transform of a Cosine Wave................................................ 30

Figure 3.10 - Change in Power Spectral Density over Time [55] ................................ 31

Figure 3.11 - Calculating time lag of a signal with the cross correlation function [71] .. 32

Figure 3.12 - Correlation coefficient as function of signal offset [72] ......................... 32

Figure 3.13 - Applying the Cross-Correlation Method to Find the Leak in a Pipe [46]... 33 
Figure 3.14 - Raw acoustic data from all sensors during normal operation 34

Figure 3.15 - Windowed FFT plot of normal pipeline operation 35

Figure 3.16 - Windowed FFT (averaged frequencies) plot of normal pipeline operation 35

Figure 3.17 - Acoustic sensor data while $1 / 4$ " hole leak 36

Figure 3.18 - Windowed FFT (averaged frequencies) plot of leak.............................. 37

Figure 3.19 - Time offset of leak signal by cross-correlation $\left(t_{0}=20.0 \mathrm{~s}, \mathrm{t}_{1}=20.1 \mathrm{~s}\right) \ldots \ldots . .37$

Figure 3.20 - Time offset of leak signal by cross-correlation $\left(t_{0}=40.0 \mathrm{~s}, \mathrm{t}_{1}=40.1 \mathrm{~s}\right) \ldots \ldots . .38$

Figure 3.21 - Acoustic sensor data while 1/8" hole leak.............................................. 40

Figure 3.22 - Windowed FFT (averaged frequencies) plot of 1/8" hole leak ................. 41

Figure 3.23 - Acoustic sensor data while bypass valve opened ................................ 42

Figure 3.24 - Windowed FFT (averaged frequencies) plot of bypass valve opened ........ 42

Figure 3.25 - Acoustic sensor while during main valve partially closed ...................... 43

Figure 3.26 - Windowed FFT (averaged frequencies) plot of main valve closure ........... 44

Figure 3.27 - Raw acoustic data from all sensors during hammer strike....................... 45

Figure 4.1 - Acoustic Transducer Anomaly Detection MLM Architecture .................... 51

Figure 4.2 - MLM model validation on ball bearing acoustics data ............................. 53

Figure 4.3 - MLM model tested with pipeline acoustics data ................................... 54

Figure 4.4 - FFT applied to acoustic signals for preprocessing ................................ 55

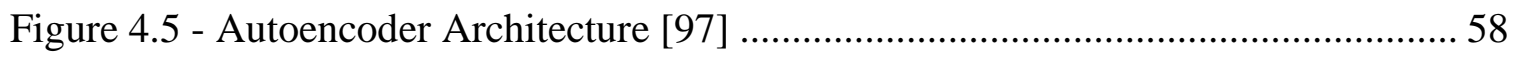

Figure 4.6 - History of autoencoder recreation error per training epoch ........................ 60

Figure 4.7 - Distribution of autoencoder recreation error of training data ..................... 61

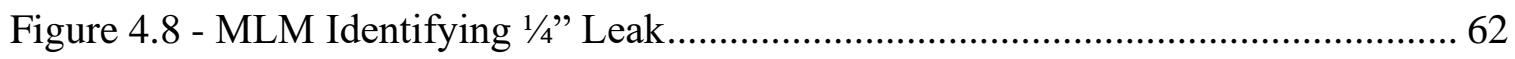

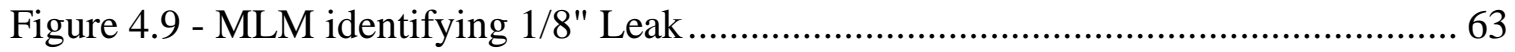




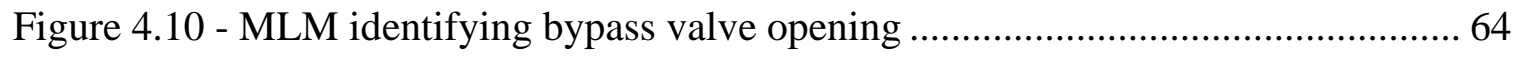

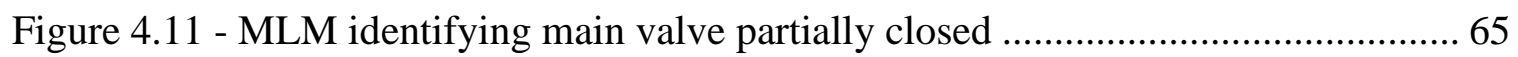

Figure 4.12 - MLM Identifying abnormal behavior while tank empties ....................... 65 


\section{LIST OF EQUATIONS}

EQUATION

PAGE

Equation 1 - Relative distance from sensors to leak ................................................ 33

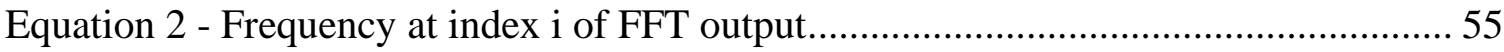

Equation 3 - Maximum number of features in array k........................................... 56

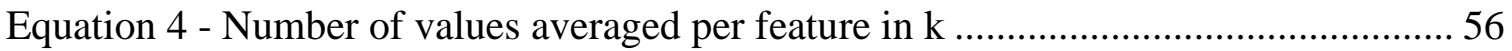

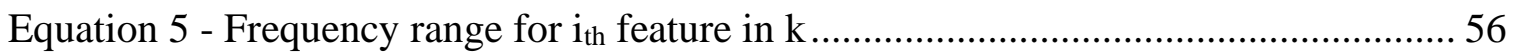

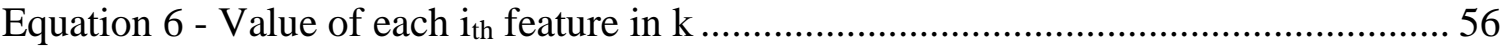




\section{LIST OF ACRONYMS AND ABBREVIATIONS}

\begin{tabular}{|c|c|}
\hline $\mathrm{ADC}$ & Analog to Digital Converter \\
\hline ARC & Applied Research Center \\
\hline ASME & American Society of Mechanical Engineers \\
\hline CEL & Cleveland Electric Labs \\
\hline CFD & Computational Fluid Dynamics \\
\hline CNN & Convolutional Neural Network \\
\hline CSV & Comma Separated Values \\
\hline DFT & Discrete Fourier Transform \\
\hline DOE & Department of Energy \\
\hline $\mathrm{E} / \mathrm{CRC}$ & University of Tulsa Erosion / Corrosion Research Center \\
\hline EMI & Electromagnetic Interference \\
\hline FFS & Fitness-For-Service \\
\hline FFT & Fast Fourier Transform \\
\hline FIU & Florida International University \\
\hline FOS & Fiber Optic Sensor \\
\hline IEP & Intrusive Erosion Probe \\
\hline LSTM & Long-Short Term Model \\
\hline MAE & Mean Absolute Error \\
\hline MLM & Machine Learning Model \\
\hline NDT & Non-Destructive Testing \\
\hline PSD & Power Spectral Density \\
\hline RNN & Recurrent Neural Network \\
\hline
\end{tabular}




$\begin{array}{ll}\text { SHM } & \text { Structural Health Monitoring } \\ \text { SPPS } & \text { Sand Production Pipe Saver } \\ \text { SRNL } & \text { Savannah River National Laboratory } \\ \text { TMI } & \text { Three Mile Island Nuclear Generation Station } \\ \text { UDP } & \text { User Datagram Protocol } \\ \text { UT } & \text { Ultrasonic Transducer } \\ \text { WRPS } & \text { Washington River Protection Solutions }\end{array}$


1 Introduction

\subsection{Structural Health Monitoring}

The American Society of Mechanical Engineers (ASME) developed a standard, Fitness-For-Service (FFS) [1], for the purpose of evaluating the structural health of components such as pipes, pumps, tanks, and valves. It covers both present integrity and the projected remaining life. Guidelines for qualitative and quantitative data are also provided for establishing remaining life, environmental compatibility, and in-service margins for continued operation. Washington River Protection Solutions (WRPS) has implemented their own fitness-for-service program, based on the AMSE standard, to evaluate the condition of tanks at the DOE's Hanford site [2]. This shows that structural health and integrity in the nuclear industry can methodically be assessed once enough accurate data is collected for a given component of interest. Much of data collection in SHM is done while systems are offline but there is growing need for real time monitoring in the nuclear industry as even small structural failures can lead to catastrophic disasters. With the development of fundamental axioms of structural health monitoring [3], the road is paved for permanent sensors and automated programming models to take much of the burden of SHM away from humans.

\subsection{Motivation}

Sensors are becoming smaller, cheaper, and more versatile as technology progresses. This has led to them being integrated into everything imaginable from roads to humans. In addition, the unique configurations of distributed sensor systems and the data processing 
that supports them is advancing rapidly as well. One group that stands to gain significantly from the advancement of sensor technology is the nuclear industry. Support for this argument is represented by the fact that seven nuclear power plants in the United States have shutdown permanently since 2013 [4], mostly due to high operating costs related to stringent regulations and expensive structural maintenance. These economic pressures have led to the DOE's continuous investigation of new types of sensors for applications in harsh environments such as nuclear power plants and nuclear waste facilities [5]. By advancing the capabilities of sensor systems, the role humans play in the collection data during routine inspections can be heavily reduced thus reducing costs, risks, and errors.

Many of the preventative measures currently taken by DOE facilities to mitigate the risk of failure include regularly scheduled maintenance and manual inspections of components such as pumps, pipes, and tanks. Relying on humans to conduct this work leads to high risks to workers. Several studies suggest that exposure to low-dose ionizing radiation at levels far below the regulated annual maximum may increase ones risk of cancer [6] [7]. In addition, relying on humans for critical process control and monitoring only increases the errors introduced into the data collection, analysis, and decision making process At the Three Mile Island - Reactor 2 (TMI-2) incident, thousands of gallons of reactor coolant were inadvertently pumped from the reactor plant before operators discovered abnormal pump operation [8]. This led to the auxiliary sump to overflow and radioactive water to back up through drain floors [8]. Operator error is widely known to be a root cause of the meltdown at Chernobyl and the same nearly occurred at TMI for the same reasons. To avoid repeating accidents of the past researchers have strived to develop data analytics models to predict, prevent, and detect system failures, however, routine 
periodic inspections no longer provide enough data to gain the major benefits of more advanced SHM models. Through permanent sensor installations designed for online monitoring, gaps in data collection can be avoided.

Acoustic sensors are particularly well suited for the needs of structural health monitoring and are currently used for many different sensing applications in the oil and gas industry [5]. The use of ultrasonic transducers (UT) are common for NDT thickness measurements of materials such as pipe walls or concrete, along with profile detection. Acoustic sensors such as conductive microphones can passively monitor equipment from a distance and detect anomalies in vibration that have signatures that develop over long periods of time and would normally be inaudible to humans and undetectable by periodic inspection. Fiber optic sensors - which can replace most classical electrical sensors - have the added immunity to electromagnetic interference and extremely low latency and no electrical power requirements [9]. Unfortunately, most sensors available on the commercial market have not yet been shown to meet the requirements of the DOE and are therefore an expensive and risky option for the facilities to adopt. By investigating existing sensor technologies for the nuclear industry this work hopes to validate different data collection approaches that would be easily deployable. In addition, an MLM based anomaly detection program is that utilizes these sensors and validates their ability to work in a real-time pipeline monitoring application that would be of value to the DOE and nuclear industry.

\subsection{Objective}

The object of this thesis is to validate the feasibility of sensor system topologies for DOE facility environments that will increase SHM capabilities while reducing costs and risk to workers. Research for this thesis can potentially be used as a guideline for adopting 
new technologies for sensing applications in the nuclear industry. This study will attempt to address the following questions with regards to SHM:

1. What available technologies exist that will be suitable for deploying in the harsh and possibly radioactive environment of the nuclear industry to facilitate real-time remote monitoring?

2. Will a long short-term memory (LSTM) machine learning model be able to effectively discriminate pipeline structural failures and process control events, utilizing the sensor systems described in this research?

\section{In Situ Coupon and UT Probe System}

\subsection{Introduction}

Transporting caustic abrasive slurries through pipelines leads to rapid erosion and corrosion. Consistent maintenance and monitoring are required to identify potential failures before they occur, incurring high repair costs and even higher remediation costs should equipment fail during operation. Pipe wall thickness measurement with ultrasonic transducers is a common practice and can provide useful insights into the rate of inner surface loss [10]. NDT techniques alone may work well for narrow scope applications, however, a single method alone is usually not enough to gain enough information about the nature of an issue [11]. To augment the capabilities of UT thickness measurement beyond wall thickness measurement, some techniques incorporate other NDT sensors or intrusive probes.

Savannah River National Laboratories (SRNL) has developed a novel approach combining intrusive erosion probe (IEP) coupons with ultrasonic measurement techniques 
to improve the accuracy and range of metrics that can be collected from active pipe infrastructure [12]. By inserting a replaceable coupon of the same material as the pipe the SRNL coupons provide a controlled environment on which the corrosive and erosive properties of the material being transported through the pipe system can be quantified and qualified. In nuclear waste facilities it is difficult to accurately predict the effects that unknown waste slurries will have on a pumping system. Quantifying erosion rates of an unknown substance on an existing and worn pipe system yields several errors in data collection that are hard to offset when taking measurements outside of a laboratory. The coupons act to remove the physical condition of the pipes as a variable when determining the erosion rate of the unknown material. The added geometry of the coupon for the fixed placement of a UT probe provides a stable environment where the same measurement location and probe orientation can be guaranteed each time a new measurement is taken.

The purpose of this research was to test and demonstrate that the SRNL erosion and mass loss coupons could provide an in-situ method for collecting erosion and mass loss rates from a pipeline during operation. The application of these coupons was experimentally tested in a bench scale pipe loop circulated with sand-water slurries of varying densities and grits. The intended advantages of the replaceable coupons is the ability to easily calculate mass loss and to also gain insights into qualitative data such as erosion patterns on the inside of the pipes [12]. This research was limited to conducting thickness measurements with a single handheld UT probe for all coupons, however, the coupons are discussed as a possible means for real-time monitoring should they be outfitted with permanent UT probes to constantly relay readings. 


\subsection{Related Works}

The study of erosion in industrial piping systems has been thoroughly researched over the years [13] [14] [15] [16]. Many techniques have been developed for monitoring the erosion of pipes and most focus on NDT methods for determining wall thickness such as radiography [17], electromagnetic acoustic transducers and eddy currents [18], and ultrasonic transducers. These methods are not typically employed while the pipeline is operational and only done periodically.

Further research has been performed on modeling pipeline erosion as predicting solid particulate erosion is crucial for operations in the oil and gas industry [19]. One of the most comprehensive erosion rate prediction software Sand Production Pipe Saver (SPPS), is based on models developed and maintained by the University of Tulsa Erosion/Corrosion Research Center (E/CRC) [20]. The software is capable of accurately modeling different liquids, gases, slurry materials, pipe materials, pipe diameters, flow rates, pipe geometries, abrasive particle sizes, and more [20]; predictions generated by the software have been validated by experimental data [19]. But even with these tools erosion still presents a significant issue to industrial piping systems and there are still many gaps in knowledge especially on some of the physical properties of the erosion phenomenon [21].

Beyond NDT, another common industry practice involves intrusive probes inserted into the pipe to be subjected to the same conditions as the internal pipe walls. IEP are typically designed for long-term pipeline monitoring [13] and may be in the form of a coupon, sensor, or probe. A hole is typically drilled in the pipe wall or a special fitting is used and the IEP is fixtured in place on the pipe. Electrical resistance probes are the most common form of IEP and measure erosion by the change in electrical properties of the 
sensing element as material is worn away [22]. Electrical resistance probes may experience unusual erosion behavior especially in more extreme conditions due to uneven erosion of the probe [23].

\subsection{Background}

The general function of an ultrasonic thickness gauge is based on the pulse-echo principle [24]. Figure 2.1 shows the process by which an ultrasonic transducer emits a high frequency pulse into the pipe wall and records the echo as the signal reflects off the two opposing surfaces of the wall. By calculating the time between the two echoes and multiplying by the propagation velocity of sound through the pipe wall material, the thickness of the wall can be determined. The delay lines act as a medium between the transducer and the pipe wall for the initial pulse to propagate through and so that there is enough time between the pulse and echo to avoid interference between the two signals.

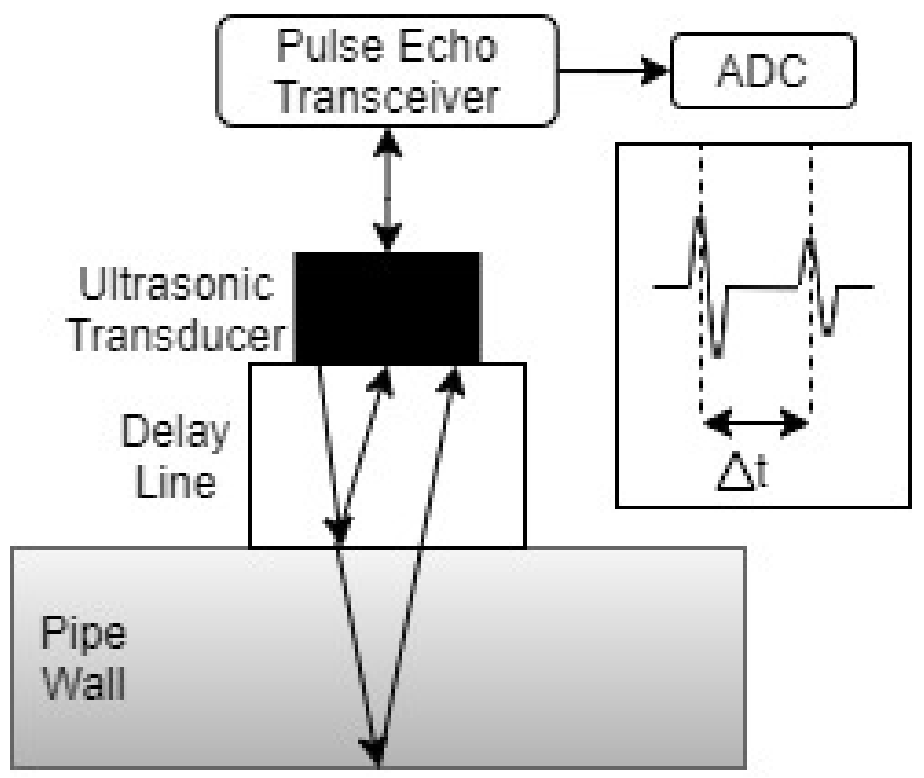

Figure 2.1 - Ultrasonic Thickness Gauge Diagram 
Errors occur in this method primarily in the measurement of time between receiving the echoes from the top and bottom surface [13]. Factors that can lead to this calculation error can be caused purely by the digital system such as clock jitter, low ADC resolution, or sampling rate. Signal noise, caused by the material or operator error, cannot always be solved by merely improving the design of the NDT probe and data acquisition system. Degradation of the pipe wall material can lead to inconsistent readings as oxidation or cracks can cause noise or multiple echoes returning from the initial pulse that confuse the digital signal processing algorithms. Poor placement of the probe, alignment, or lack of couplant can result in similar issues.

Handheld UT probes that a technician can use to perform numerous inspections in any accessible area on a pipe are more cost-effective solutions than permanently mounted sensors. A permanently mounted UT sensor is limited to taking point measurements and many must be placed at varying intervals to capture an accurate snapshot of wall thickness along a given stretch of pipe. The benefit of permanently mounted probes is that they usually have higher accuracy and higher rate of reproducibility due to the amount of care taken during installation and lack of movement; a site on the pipe must be prepared and often the sensors are calibrated each time they are mounted, and will not change position again until uninstalled. Handheld probes are subject to more reading errors due to poor conductivity between the probe and the pipe, misalignment, and other forms of operator error.

The coupon system validated in this chapter is designed to address the issues presented with handheld probes and degraded pipe walls. The coupons are designed to be mounted on a pipe and provide a controlled environment in which a handheld UT probe can get 
highly accurate and reproducible thickness measurement readings. After any period of installation, the coupons then have the added benefit of being removeable so that the inner surface can be inspected to qualify the erosion process happening inside the pipes without the need for sectioning.

\subsection{Materials and Methods}

\subsubsection{Sensor System}

The SRNL coupon system is designed to provide the ability to measure extremely small values of mass loss, surface roughness profile, and thickness changes, while not disrupting the flow inside the pipe or stopping normal operation. A modified Swagelok fitting used to hold the coupon in place is first welded perpendicular to the pipe surface shown in Figure 2.3, then a hole approximately $5 / 16$ " is drilled through the pipe in the center of the Swagelok fitting; this allows for the coupon head to be placed flush with the inner surface of the pipe. The replaceable coupon is designed to be inserted into the holder with a polymeric sleeve to isolate the coupon from vibration which in turn will reduce fretting and improve UT probe readings. The coupon holder also plays the role of electrically isolating the coupon from the piping system, which if not isolated may cause unwanted galvanic effects between pipe and coupon. The coupon itself can be scaled for different pipe sizes or applications. 


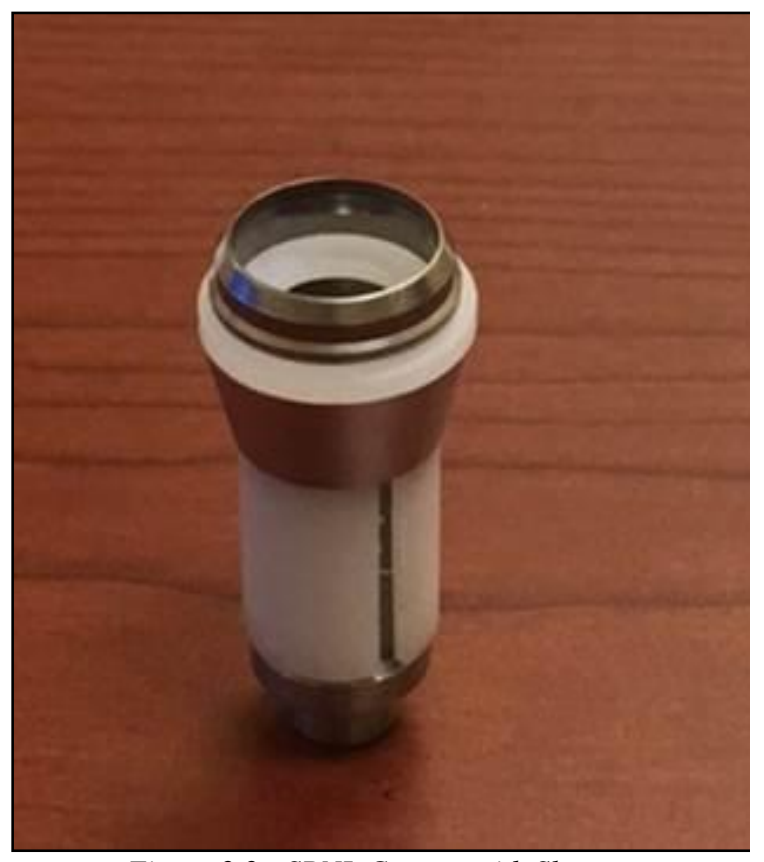

Figure 2.2 - SRNL Coupon with Sleeve

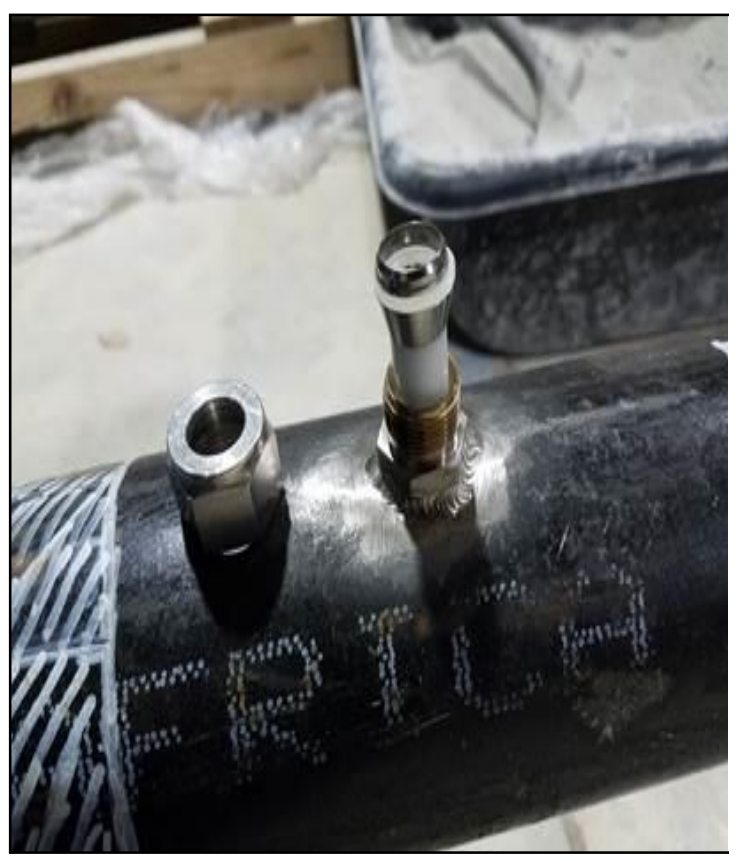

Figure 2.3 - Coupon Inserted into coupling affixed to pipeline

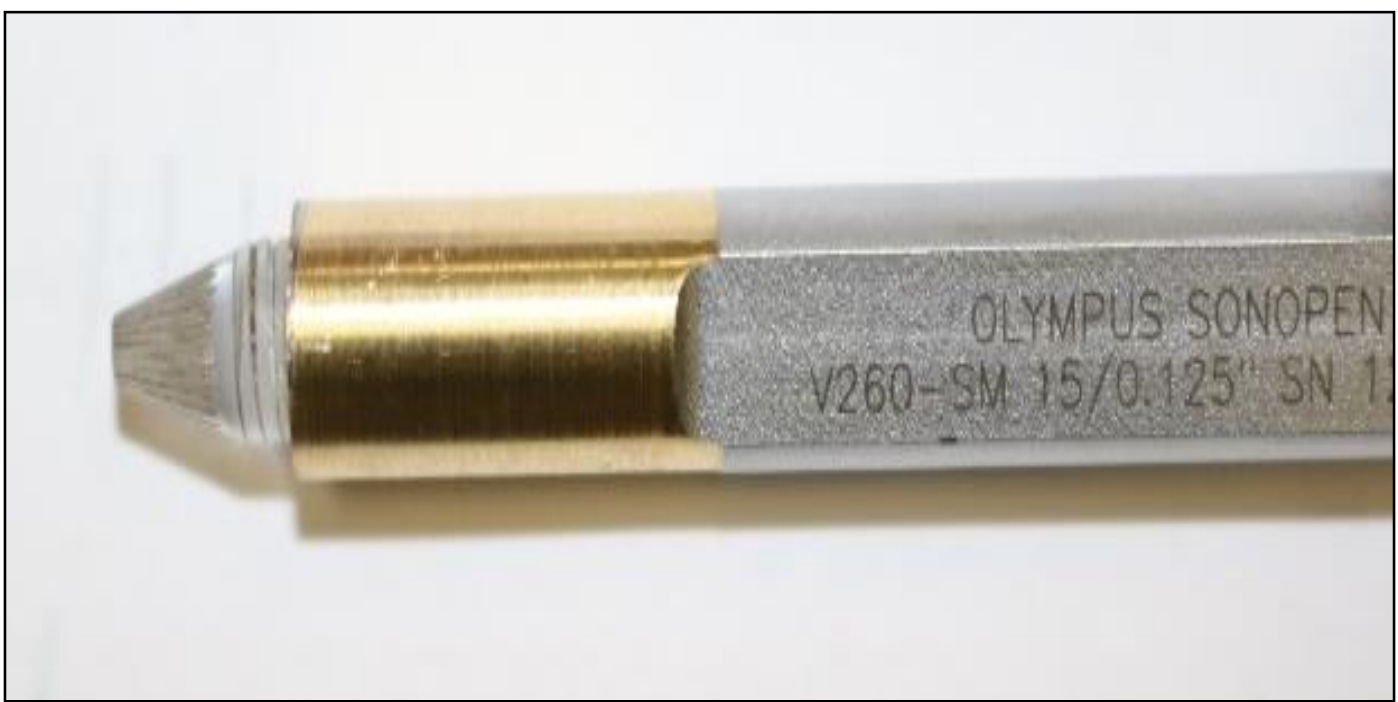

Figure 2.4 - Modified Olympus V260-SM UT Pencil Probe

Use of the replaceable coupon provides for a controlled location for measurements taken by an ultrasonic probe, providing a guide that ensures repeatable probe head placement and orientation for each measurement. In addition, the ease of removing of the coupon allows for measurements and inspections to be conducted away from the pipe loop 
and with tools and techniques that would otherwise not be possible. Mass loss can be determined with a balance of high precision $(.00001 \mathrm{gm})$ and height change can be measured with a micrometer. Pit morphology and scar profile can be documented and inspected as well. These features could lead to shorter duration wear tests or provide insights into wear in systems where modeling and traditional methods are ineffective. In order to take highly accurate UT probe readings, an off the shelf UT pencil sensor (Olympus V260-SM) probe was modified to fit the inner channel of the coupon [25], shown in Figure 2.4. The head of the UT probe was turned on a lathe and reduced to ensure a snug fit into the coupon. The inner channel of the coupon provides a guide for the UT probe so that for each measurement, the head of the sensing element always touches the same point on the coupon and maintains a near perfect perpendicular angle with the surface. By ensuring the UT probe is guided, placement error can be avoided, and reproducibility of measurements can be guaranteed.

\subsubsection{Experimental Design}

To simulate the effects of waste on degradation of a pipeline system, a bench scale pipe loop was constructed of commonly used geometries found in DOE nuclear waste facilities $-50.8 \mathrm{~mm}$ ( 2 inch) and 76.2 $\mathrm{mm}$ ( 3 inch) carbon steel pipes, elbows, and reducers. An effective abrasive mixture was formed by combining water and sand -50\% (20/30 grit) and $50 \%$ (35/60 grit) - 10\% V/V. The mixture was then circulated through the loop using a 3 Horsepower (HP) pump drawing from a reservoir where the sand mixture was continuously agitated using a concrete mixing head. The pipe loop then emptied its return to the same reservoir so that the sediment may stay in constant circulation during the entire

duration of the test. Five coupons were installed on the pipe loop on a $76.2 \mathrm{~mm}$ (3 inch) 
straight section, 3 coupons placed directly on top and 2 placed directly below, all spread between a 0.6 -meter ( 2 foot) length.

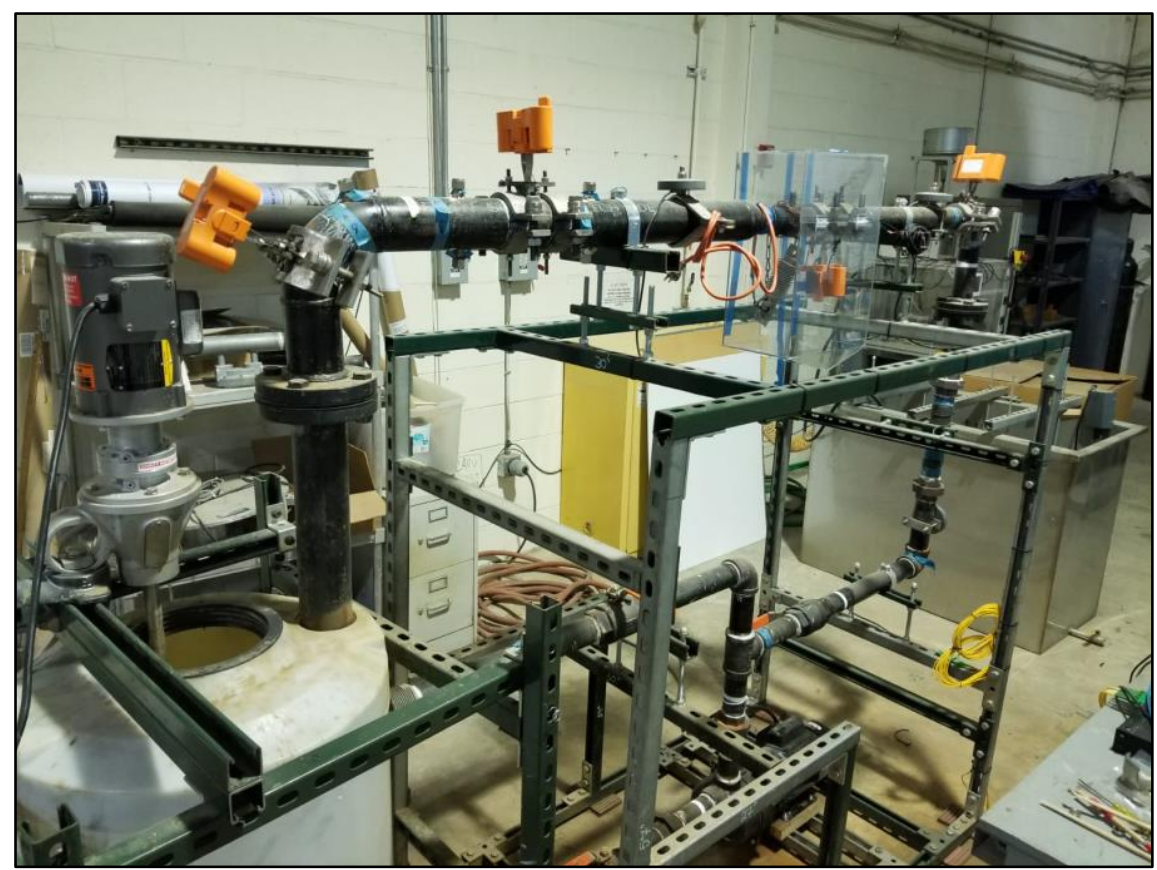

Figure 2.5 - Bench Scale Pipe Loop Setup

To quantify the erosion taking place in the pipe, the coupons were weighed and measured using a balance and caliper before being inserted. The surface of the coupon head was also viewed under a microscope and photographed for later comparison after erosion had occurred. During the test, measurements were taken in situ using an Olympus V260SM UT pencil sensor that was modified to fit specifically in the provided channel within the coupon. Thickness measurements were collected every 30 minutes from both the UT pencil sensor and the UT sensors permanently mounted. After testing, the coupons were removed from the pipe loop and measurements were taken again to quantify mass and height loss, and validate the measurements taken by the pencil sensor. 


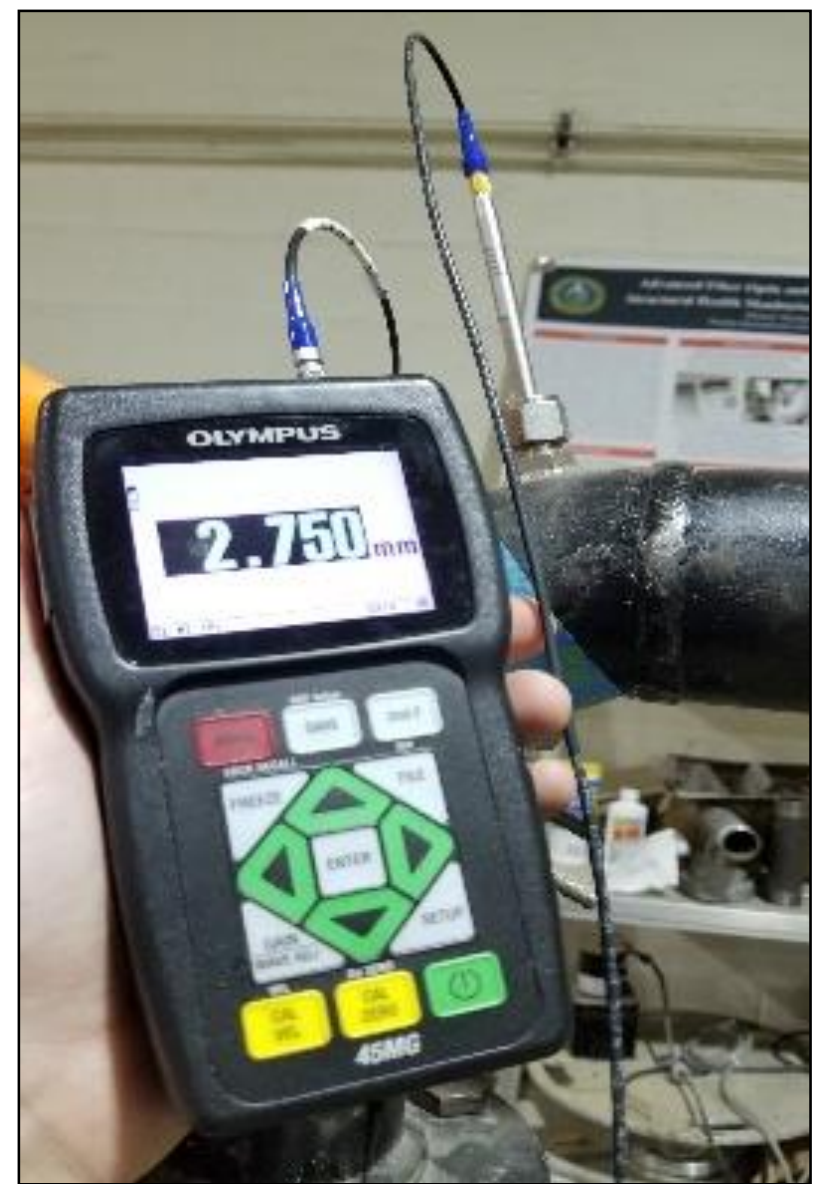

Figure 2.6 - Collecting UT probe reading from installed coupon

\subsection{Results and Discussion}

Surface profile changes for the five coupons involved in the test can be seen in Figure 2.7. The sand slurry was circulated through the pipe loop for approximately 7 hours and measurements were taken before and after. It should be noted that coupons 1-3 were placed on the top of the pipe section and coupons 4-5 were placed on the bottom directly below. The surface of the new coupon after being turned on a lathe can be seen in Figure 2.7 a), representing the starting surface condition of all five coupons used in the test. It is extremely noticeable that the coupon heads exposed to the pipe ceiling (1-3) all exhibit a similar wear pattern: long and deep surface scaring; coupons placed on the bottom of the 
pipe (4-5) exhibit a much more uniform erosion pattern and none of the deep scar characteristics of coupons place on the pipe ceiling.

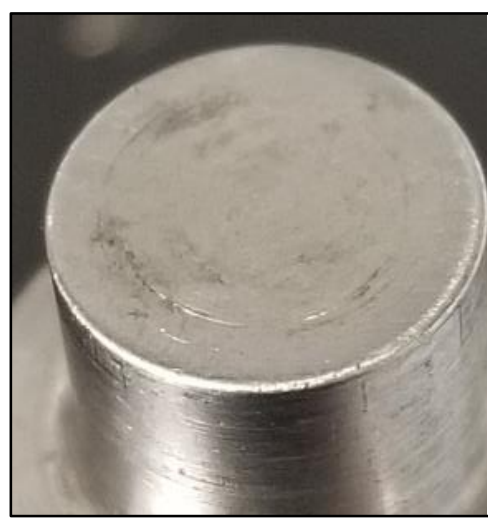

a) New Coupon

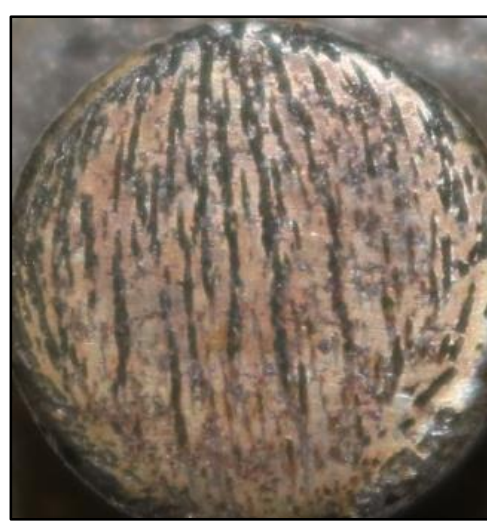

d) Coupon 3

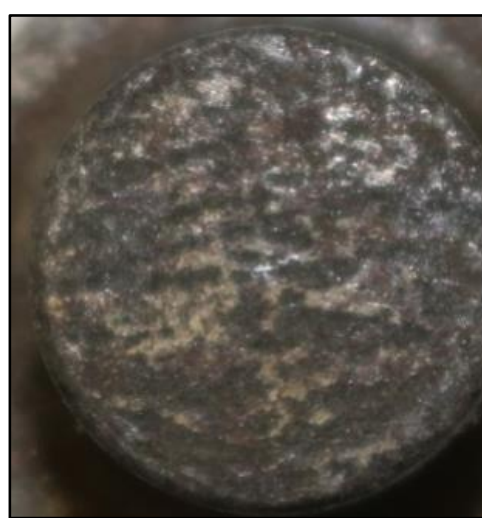

b) Coupon 1

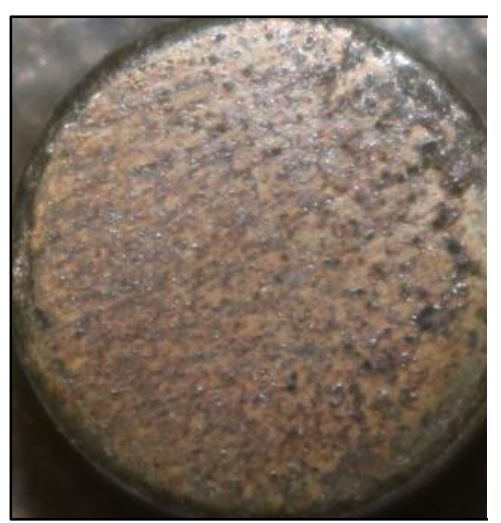

e) Coupon 4

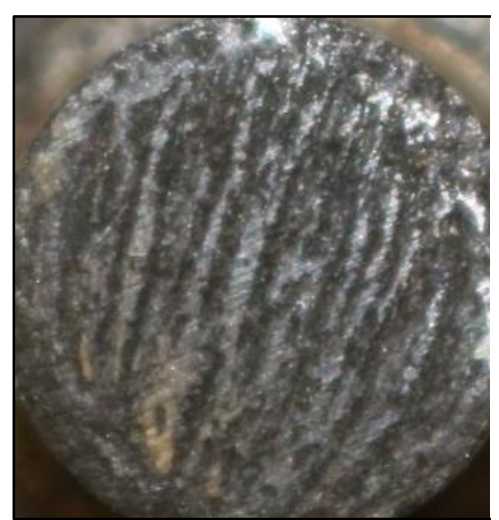

c) Coupon 2

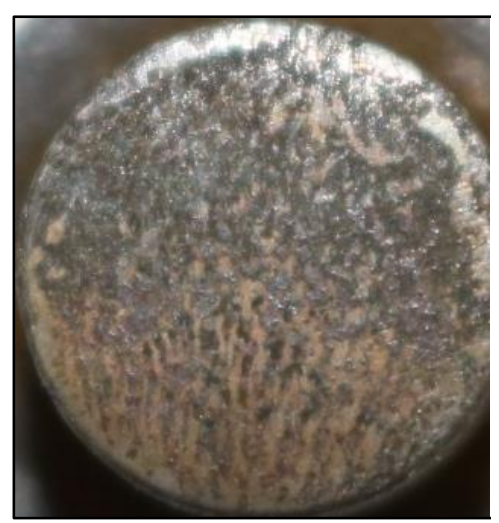

f) Coupon 5

Figure 2.7-SRNL Coupons' Eroded Surfaces

The current hypothesis for the uniquely different forms of erosion is that sediment particle velocity plays the largest role. While traversing the pipeline horizontally, sediments tends to settle on the floor of the pipe and move at velocities less than the nominal rate of flow in the pipe. In addition, a higher percent of sediment settling covers a larger surface area many particles thick. This results in a continuous sanding of pipe wall at lower speeds. Evidence of this can be seen in the smaller pit diameters and depth, and the more uniform coverage of erosion. Meanwhile, particles impacting the ceiling of the pipe may be carried 
in currents of turbulent flow, accelerating particles at speeds greater than the nominal flow. In addition, because the density of the particles (sand) is much greater than that of the medium (water), and the particles do no exhibit any coagulating properties, the ceiling of the pipe is subjected to individual particle strikes at high speed. Particles introduced into the same streams of turbulent flow may follow a similar trajectory, subjecting certain areas of the pipe surface to high levels of erosion while neighboring regions experience little erosion. Evidence for this can also be seen in the microscopic images. Surface scars are long and deep, however, between scars there appears to be much less relative erosion.

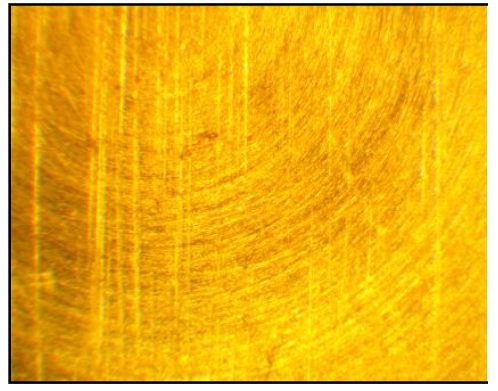

a) New Coupon

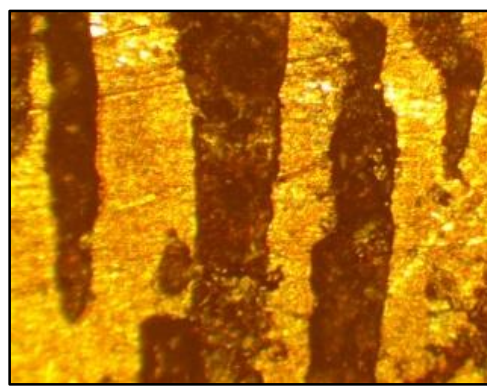

d) Coupon 3

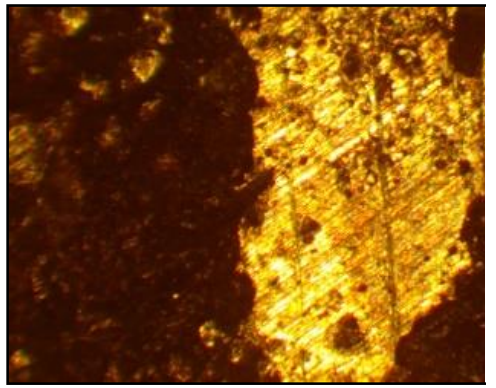

b) Coupon 1

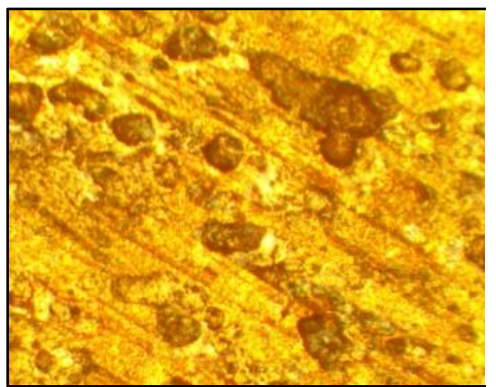

e) Coupon 4

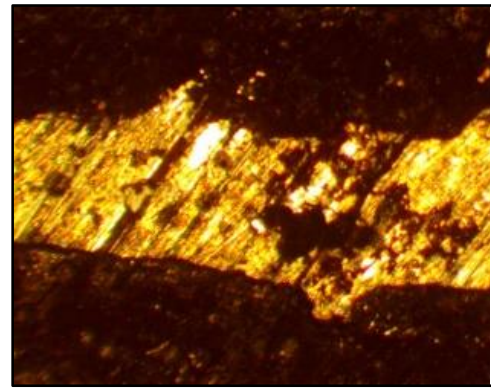

c) Coupon 2

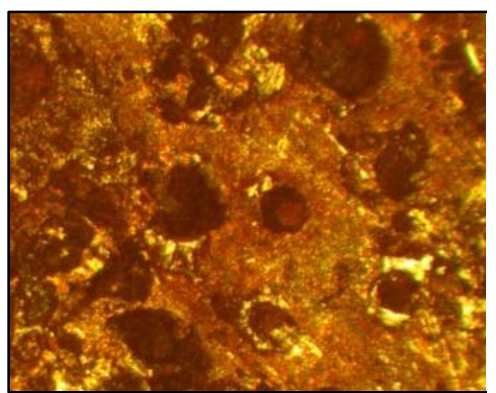

f) Coupon 5

Figure 2.8 - Erosion Patterns of Coupon Surfaces Under $5 X$ Magnification

After testing, erosion was quantified by taking mass and height measurements. As expected, there were significantly different changes for erosion taking place on the pipe floor as opposed to the pipe ceiling. In Table 1, the height change, and mass loss of the coupons can be seen. Notably, coupons on the ceiling exhibited lower mass loss but higher 
height loss, whereas the coupons mounted on the pipe floor exhibited higher mass loss and lower height change.

\begin{tabular}{|c|c|c|c|c|c|c|}
\hline \multicolumn{7}{|c|}{ SRNL Coupon Height and Mass Loss Measurements } \\
\hline Coupon \# & $\begin{array}{c}\text { Starting Mass } \\
(\mathbf{g m})\end{array}$ & $\begin{array}{c}\text { Starting } \\
\text { Height }(\mathbf{m m})\end{array}$ & $\begin{array}{c}\text { Ending Mass } \\
(\mathbf{g m})\end{array}$ & $\begin{array}{c}\text { Ending } \\
\text { Height (mm) }\end{array}$ & $\begin{array}{c}\text { Mass } \\
\text { Loss (gm) }\end{array}$ & $\begin{array}{c}\text { Height Loss } \\
(\mathbf{m m})\end{array}$ \\
\hline 1 & 7.872 & 25.50 & 7.868 & 25.46 & 0.004 & 0.04 \\
\hline 2 & 8.469 & 25.79 & 8.462 & 25.76 & 0.007 & 0.03 \\
\hline 3 & 7.691 & 25.51 & 7.683 & 25.48 & 0.008 & 0.03 \\
\hline 4 & 7.76 & 25.50 & 7.753 & 25.48 & 0.007 & 0.02 \\
\hline 5 & 7.851 & 25.44 & 7.835 & 25.42 & 0.016 & 0.02 \\
\hline
\end{tabular}

Table 1 - SRNL Coupon Mass and Height Loss

In addition to the measurements detailed in Table 1, the Olympus UT probe was also used to take measurements in situ during the test. After comparing the results of the UT pencil sensor to caliper readings taken after testing, it can be shown that the accuracy of using the UT pencil probe during operation can yield thickness measurement changes accurate to $.025 \mathrm{~mm}(.001$ ") and a reproducibility of measurement on the same coupon of $.0127 \mathrm{~mm}($ $.0005 ")$.

\subsection{Conclusion}

Current research verifies and validates the use of the SRNL coupons on an engineering scale test bed. From the data obtained from the SRNL coupon testing, the erosion rates of the ceiling versus the floor of the pipe could easily be calculated after several hours of experiments. On average, the bottom of the pipe loss showed $1.6 \mathrm{mg} / \mathrm{hr}$ and $.0028 \mathrm{~mm} / \mathrm{hr}$ 
mass loss and thickness loss respectively while the ceiling of the pipe lost $0.9 \mathrm{mg} / \mathrm{hr}$ and $.0047 \mathrm{~mm} / \mathrm{hr}$. In addition, extremely valuable insights into the method of erosion were gained from the ability to remove and inspect the coupons after a test. Under typical circumstances, the thickness change measurements recorded by a UT sensor would lead to the conclusion that more mass is being lost from the ceiling of the pipe section due to the fact is has a higher average height change, however, by being able to inspect the surface of the coupons subject to wear, it is clear that average height change and mass loss are not as closely correlated. The reason could be due to the uneven surfaces resulting from particle agglomeration, settlement to severe flushing with high velocities.

Compared to conventional forms of wear testing in the field, the SRNL coupon system is vastly superior for reasons outlined in this research so far. By being replaceable, the coupons allow for accurate testing of erosion in a field environment but with the added benefit of being able to start with a fresh surface and a controlled location to take measurements. These factors alone reduce the errors resulting from improper probe angle and placement when taking measurements, not to mention surface defects and corrosion on the inner surface of the pipe that can lead to inaccurate UT measurements. By being able to remove the coupons and collect highly precise mass measurements, specific erosion quantification can be achieved while previously the assumptions on the relationship between mass and thickness change were forced to be made.

The findings of the bench scale test performed during this research indicate that the SRNL coupon system will be an impactful new technology for waste management facilities, and many other applications requiring erosion measurement as well. Further testing of longer duration is required to prove validity on larger scale systems where tests 
may last months instead of hours. Also, modifications to the current bench scale pipe loop are planned to quantify more variables such as pressure and flow rate inside the pipe. With these added values, more complex models of erosion can be determined from the quantities extracted from the coupon system. Finally, the coupons must be assessed for failure potential and expected lifetime usage. For operational pipelines that are not solely for experimental conditions, mounting the coupon system on a section of pipe must prove to be a permanently safe operation. Degradation of coupon components during normal operation and exposure to the elements must be analyzed.

\section{Fiber Optic Acoustic Transducers}

\subsection{Introduction}

Acoustic sensors, which cover a broad range of sensing devices, may either be passive or active devices that record vibrational energy traveling through a given medium. By analyzing the waveform, signatures for unique events or geometries in the effective areas can be extrapolated. Acoustic sensors are commonly applied to structural health monitoring (SHM) for crack detection in structures, as well as fault detection in pipelines [26] [5].

For SHM of piping systems in the nuclear industry electrical sensors can present some issues depending on the hazard level of the location where the sensors will be installed. Accelerometers, ultrasonic transducers, and acoustic transducers make up the backbone of SHM sensors and are well suited for long term installations for real-time monitoring. These devices, though, rely on high precision analog to digital converters (ADC) which are extremely sensitive to ionizing radiation and may result in inaccurate readings when exposed. In addition, any electrical circuit or data transmission line is susceptible to the 
effects of ionizing radiation found in nuclear sites if not shielded properly. This requires extensive radiation hardening techniques to be applied to the design of the electrical sensors and systems and rapidly raises the costs. Electrical sensors also require a continuous power source, this limits the length of the conduit as an 18 AWG copper wiring passing 1 Ampere of current will result in more than a $10 \%$ voltage drop over $100 \mathrm{ft}$. Outfitting a long section of pipe with electrical sensors would require multiple substations to maintain voltage for all sensors. Wireless battery powered sensors are feasible but have tradeoffs. Most battery powered solutions are designed to collect and transmit measurements periodically, every several hours or once per day. To continuously collect real-time information, battery powered sensors would require constant field visits by technicians to replace the batteries, and if the devices are in accessibly difficult or hazardous areas, this is less than desirable.

An alternative to classical electrical sensors are fiber optic sensors that can perform the same tasks as accelerometers [27] and acoustic transducers [28] for SHM as well as a number of other measurements such as temperature [29] and stress/ strain [30]. Due to the inherent lack of electrical energy present in passive fiber optic sensors, signals and data collection are not susceptible to corruption from electromagnetic interference (EMI) [31]. When exposed to ionizing radiation optical fiber exhibits a darkening of conduit resulting in a lower propagation of light [32] [33], however, radiation-hardened single-mode and multi-mode fiber are readily available for purchase [34] [35], thus reducing the need to create custom sensors for radiation prone environments. When outfitting many sensors to a long stretch of pipeline, fiber optic cable has the benefit of being able to carry a signal for miles without attenuation, data loss, or corruption. 
Real-time leak detection, which is of primary focus of this work, requires continuous monitoring of a system over large distributed areas and require high data rates and low latencies making fiber optic acoustic sensors an attractive option. This chapter covers the work validating the application of fiber optic acoustic transducers developed by Cleveland Electric Labs for the application of real-time pipeline monitoring.

\subsection{Related Work}

Acoustics monitoring, the measurement of sound waves propagating through a medium to evaluate characteristics of the medium or material, has been used for more than a century to inspect structures and components for damages or flaws [36] [37] [38] [39]. There are many forms of SHM techniques and equipment specifically for pipeline monitoring such as infrared thermography [40], ultrasonic guided waves [41], and process model-based techniques [42] to name a few. A specific technique for application with acoustic transducers, the cross-correlation method, is widely used for leak detection in buried pipes and has been a trusted method in the water industry for decades [43] [44] [45]. While the cross-correlation method can work in noisy environments with high signal-to-noise ratios, research has shown that noise caused by rotating machinery such as pumps and motors can be filtered out to improve results [46].

Presently, most sensors are electrically powered and transmit acoustic data via an electrical signal. The latest technology relies instead on fiber optic data transmission meaning the sensors are fed and output only a light source. This provides several advantages over classical sensors such as decreased response time, decreased interference from EMI, and the ability to carry a signal over extremely long distances without signal loss or corruption [47]. Two main forms of fiber optic sensors (FOS) are intrinsic sensors, 
in which the optical fiber itself measures certain properties such as pressure, temperature, strain, vibration, or other quantity through the modulation of the latency, polarization, wavelength, intensity, or phase of the light transmitted through the fiber [48]. Intrinsic FOS are excellent for providing distributed sensing over large areas. Extrinsic FOS use a fiber optic cable as merely a transmission line and are usually a non-fiber optical sensor or an electronic sensor using an optical transmitter [48]. Several other advantages of FOS are their resistance to environmental conditions such as pressure, temperature, and radiation compared to semiconductor devices.

Utilizing fiber optic sensors for leak detection is not a new study and has been researched for several decades [49]. Most of the research, however, is based on intrinsic optical fiber sensing [49] [50] [51] [52] and some methods even require the placement of the fiber inside the pipe [53]. Extrinsic fiber optic acoustic sensors have become more prevalent in the last decade and have shown performance levels comparable to classical piezoelectric transducers [31] with the ability of detecting infrasound from $1-20 \mathrm{~Hz}$ [54].

\subsection{Method}

\subsubsection{Sensor System}

Cleveland Electric Laboratories has developed a new extrinsic fiber optic acoustic transducer, the basis of their FiberStrike System [55]. Originally developed as a perimeter security platform for intrusion detection via ground vibrations and other noises [56], has been also been tailored for pipeline monitoring. Figure 3.1 provides a high-level architecture of the FiberStrike system for actively monitoring pipelines in real-time. 


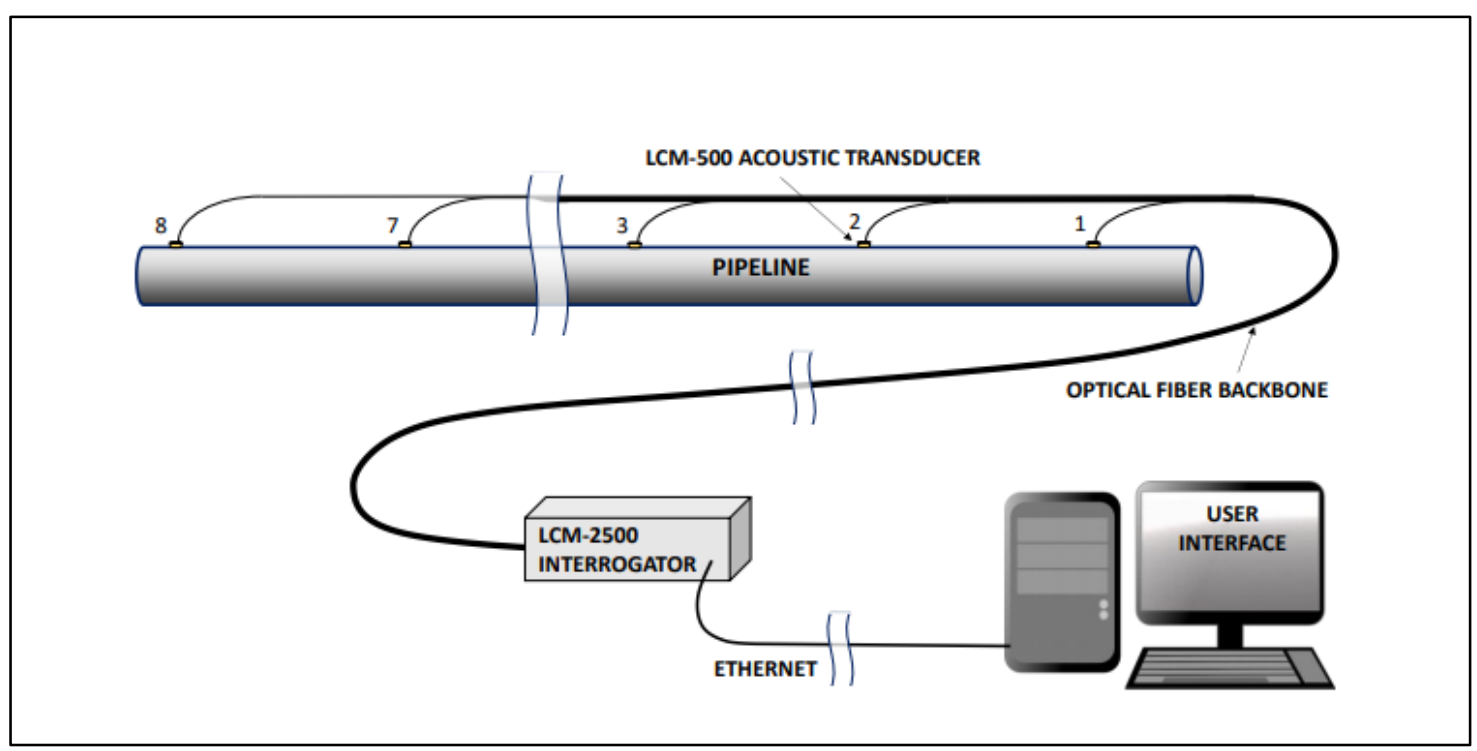

Figure 3.1 - CEL Fiberstrike Pipeline Monitoring System Architecture [55]

The system is based on an extrinsic fiber optic acoustic transducer shown in Figure 3.2. The puck shaped transducer is comprised of an aluminum diaphragm with optical fiber wound round a center core that attenuates the transmission of light through the fiber as it is subject to vibration.

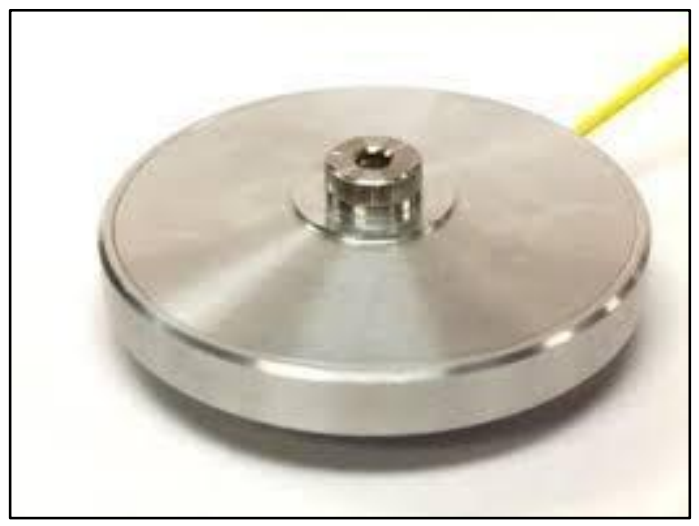

Figure 3.2 - CEL Fiberstrike Acoustic Transducer without protective cover [55]

The sensor only requires two single mode fibers for operation, one from an input light source and the second for the attenuated signal to return from. Sensors are linked via optical 
fiber to an interrogator that provides the light source and converts the optical signal to a digital output. Each interrogator can have up to 16 sensors connected with each sensor able to be $20+\mathrm{km}$ from the light source without signal degradation [55].

\subsubsection{Setup}

The experimental test setup was constructed using a pipe loop that included 50.8 $\mathrm{mm}$ (2inch) and $76.2 \mathrm{~mm}$ (3inch) carbon steel sections. Sensors were installed per the manufacturer's instructions: a threaded boss is attached to the exterior of the pipe, typically, it is physically attached to the pipe by clamping it with an industrial rated strap or clamp. The Transducer shown in Figure 3.3 is attached to the threaded boss using a supplied nut and washer.

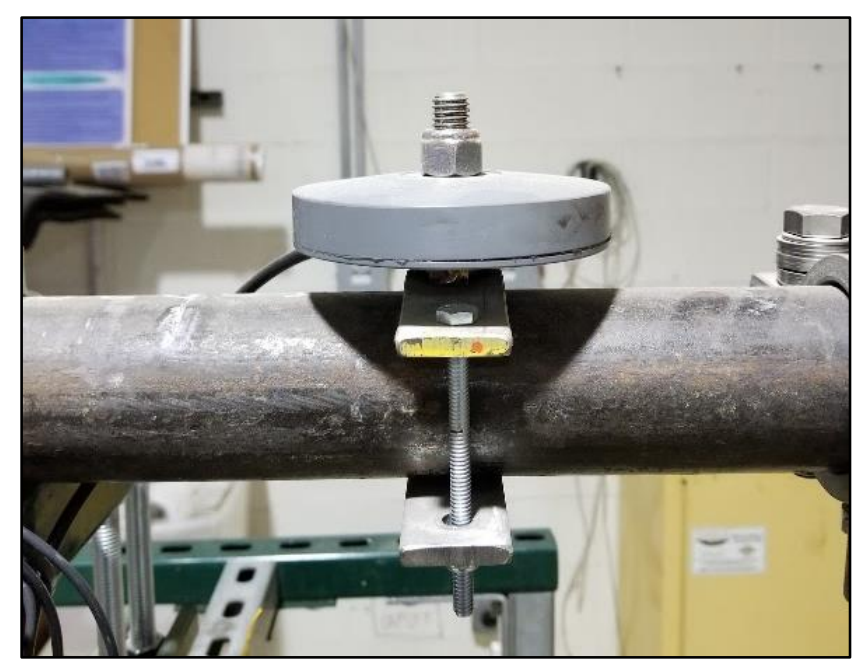

Figure 3.3 - CEL Fiberstrike Acoustic Transducer Installed on Pipe

Once installed, each sensor must have their pigtail fiber cables fusion-spliced into the fiber optic backbone cable that travels the length of the pipeline to the interrogator. Figure 3.4 shows the monitoring station placed several feet away from the scale pipeline with a laptop sitting on top of the LCM-2500 interrogator. 


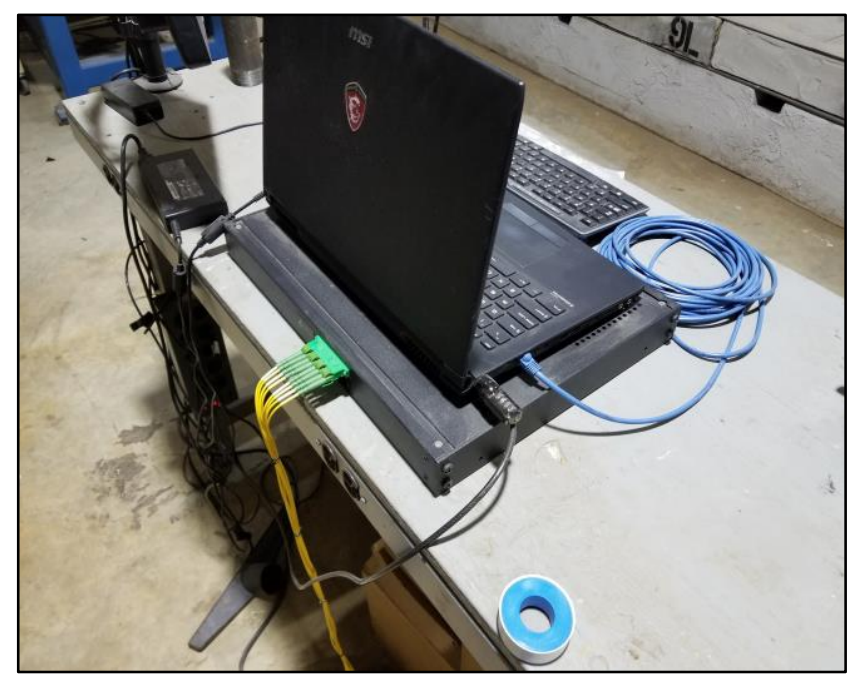

Figure 3.4 - LCM-2500 Interrogator and User Control Station

If this were a real-world scenario, and additional insulation jacket would most likely be installed around the pipe and sensors for further protection from the elements. A $3 \mathrm{Hp}$ centrifugal pump (Dayton 4YU37A) with flow rate of 110-160 GPM was used to circulate water through the pipe loop setup and an 80-gallon plastic tank was used as a reservoir to recirculate the water continuously through the system.

\subsubsection{Data Collection}

The CEL Fiberstrike system is comprised of sensors, fiber, and an optical interrogator. The LCM-2500 interrogator serves as the light source, optical interferometer, and initial signal processing unit, converting the optical signals into a UDP stream that is output over a standard ethernet connection. A laptop connected to the interrogator via ethernet runs proprietary software provided by CEL to visually display the incoming data from the FOS. 


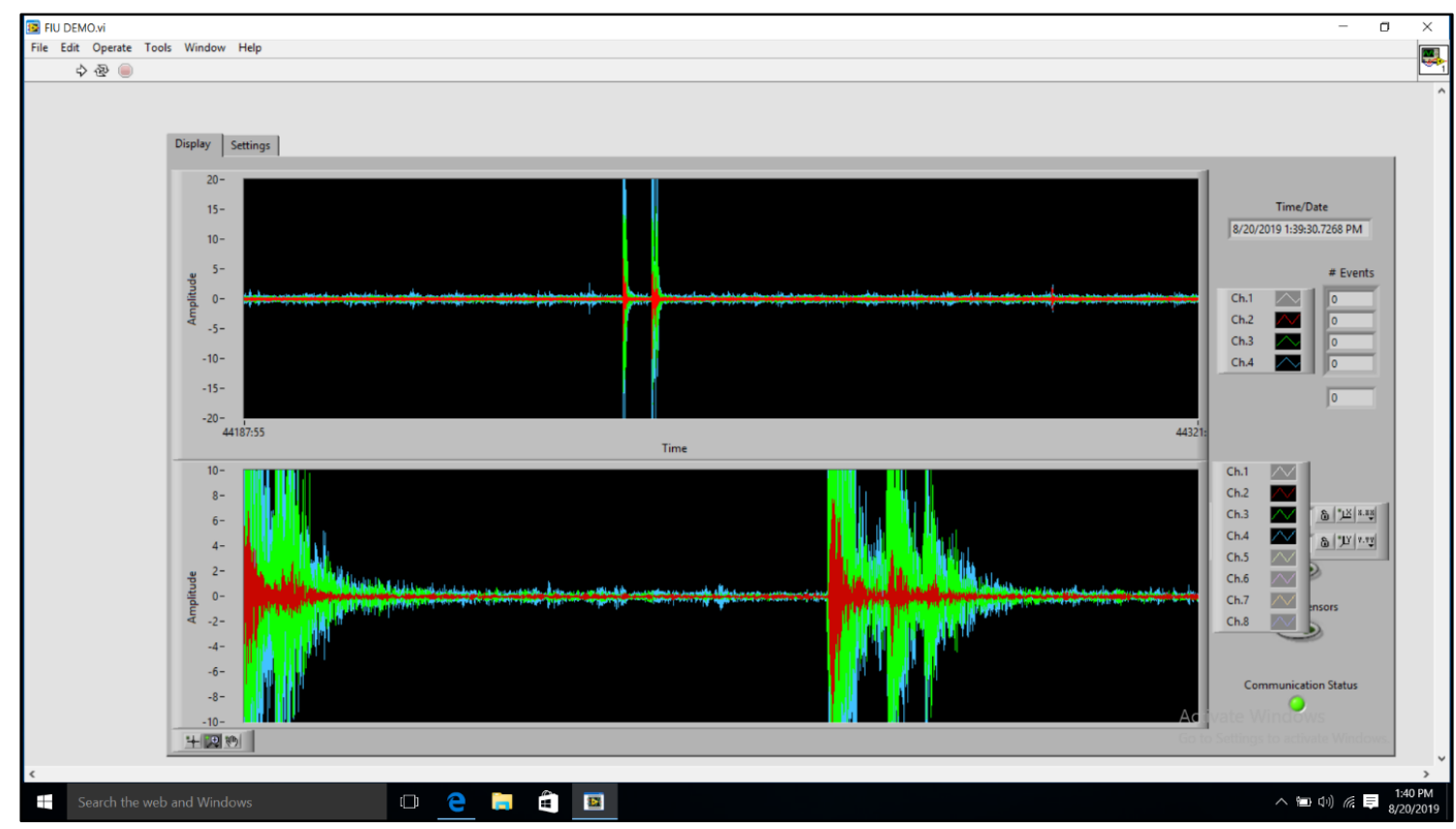

Figure 3.5 - CEL Fiberstrike Data Aquisition Software

Sensor data viewed on the program may then be exported as either images or raw data points to an excel sheet. The software was quite limited though, as data could only be exported in a limited time window of approximately 10 seconds at a time and further manipulation of the data was not possible. Therefore, an alternative means of extracting the raw sensor data was needed.

The optical interrogator streams the sensor data via UDP (User Datagram Protocol), a standard packet format used by many web applications for real-time services such as video or audio streaming [57]. Wireshark, an application for sniffing interfaces and capturing packets [58], was used to intercept the UDP stream between the optical interrogator and the proprietary software. Once intercepted, the UDP packets could then be captured for any duration of time needed and saved to file where a custom program could extract the relevant sensor data from the UDP packets and manipulate the data as needed to extract further features. 
Python was chosen as the language to develop a feature extraction program in, due to its advanced data analytics and visualization libraries such as NumPy [59], Pandas [60], and Matplotlib [61]. Another important reason was PyShark [62], a packet parsing library based on Wireshark's command line utility, tshark [63], which allows for easily dissecting the UDP packets with little efforts. Other languages such as JavaScript, C / C++, and MATLAB were considered, however, Python was chosen primarily because of its quick learning curve and thorough documentation on data analytics. While Python may not be as lean as languages such as $\mathrm{C} / \mathrm{C}++$, processing time was not a constraint since data would not be processed in real-time. Python is an excellent language for building simple proofof-concept programs; once the concept is proven and the structure of the program is determined, it would then make sense to translate it into a more efficient language such as $\mathrm{C} / \mathrm{C}++$

To reverse engineer the UDP packet structure sent by the optical interrogator, the data of the packets was compared to raw sensor data values exported by the software provided by CEL. While analyzing the characteristics of the raw data it was discovered that each data point has no more than 3 significant digits and a range of -25.0 to 25.0. This scalar value represents the uncalibrated magnitude of the acoustic signal being measured by the acoustic transducers and it should be noted that there are no units associated with it. UDP packets typically store data in hex format and it was determined after some trial and error that each raw data value was stored as a 2-byte signed integer in hex format. To account for decimal values but avoid translating a floating point or double precision decimal into hex, each value was scaled by a factor of 1000 . Through additional analysis, it was determined that each packet is formatted to transfer data from up to 16 different fiber 
optic sensors, since only 4 sensors were being used, the remaining fields were reported as zero values. In addition, each packet carries 16 sequential values for each

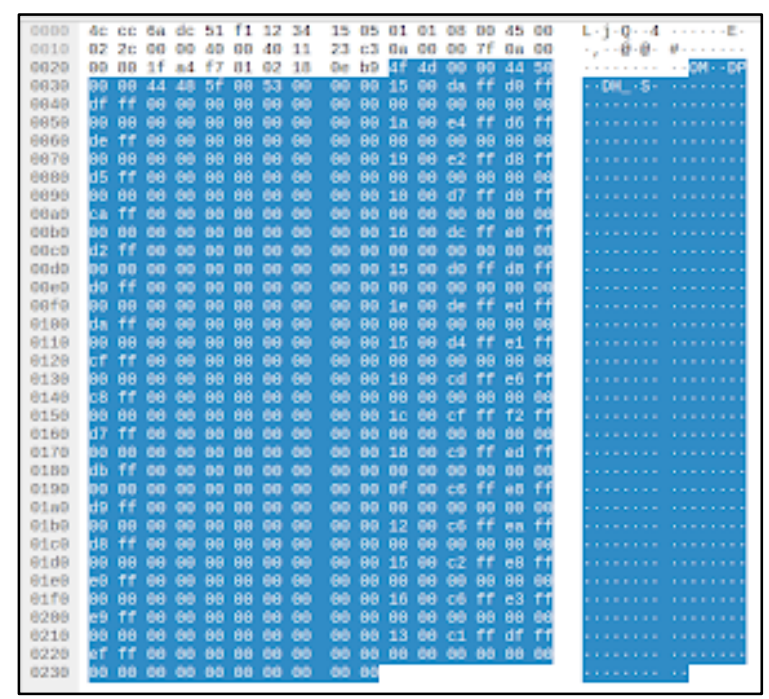

Figure 3.6 - Raw UDP Packet Data From LCM-2500

reporting sensor. Once the packet was parsed each raw value could be converted back into a signed floating point decimal with 3 significant figures by performing the two's compliment [64] algorithm and then dividing the subsequent decimal integer value by 1000. The pseudo code to represent these steps is as follows:

float rawSensorValues[16][4] //create a 2D array to hold sensor values udpPacket = importPacketCaptureFile("file/path") //import packet capture

for $\mathrm{i}$ in range(16)

\{ row = parseNextRow(udpPacket.data) //extract a row of data containing sensor values for $\mathrm{j}$ in range(4)

\{ hexValue = parseNextTwoBytes(row) //extract 2 bytes representing sensor value \} rawSensorValues[i][j] = twosCompliment(hexValue) / $1000.00 / /$ convert

\}

return rawSensorValues

Figure 3.7 - Sensor Value Conversion from UDP Packet Format 
After deriving the algorithm to convert the UDP packets into raw sensor values it became possible to record large timespans of data from the acoustic transducers and perform further analytics on the data to extract features that would be beneficial for identifying faults in the pipeline.

\subsubsection{Fault and Event Simulations}

Pipelines can suffer a number of different faults during operation and previous work has shown that unique signatures may occur depending on the type of event or fault [65]. Faults and events that are expected to cause unique changes in the pipeline's power spectral density, and therefore an acoustic signature, are:

- Leaks

- Blockages

- Valve Position

- Pump Operation Modes

- Physical Impacts to the Pipeline

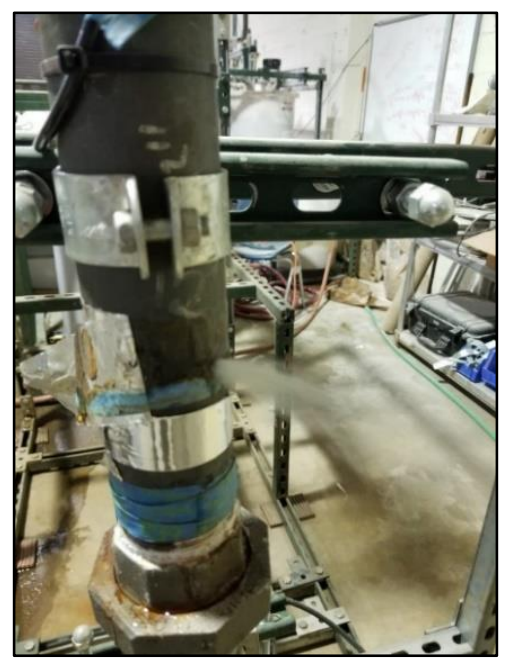

a) $1 / 4 "$ Hole

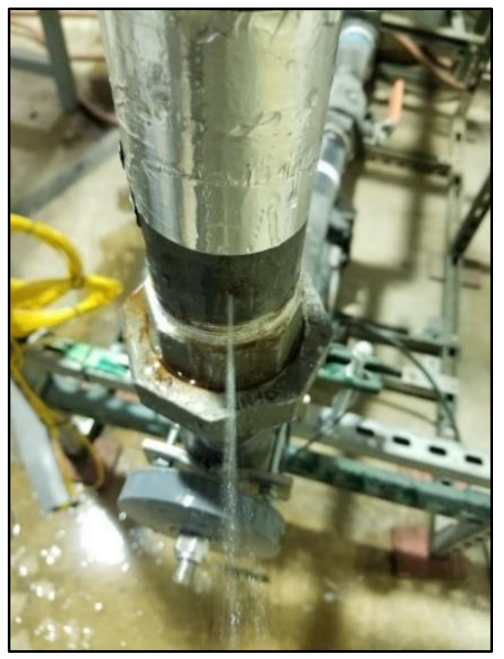

b) $1 / 8$ " Hole

Figure 3.8- Leaks Under Normal Operation 
To simulate a leak in the pipeline two holes of different sizes were drilled on the vertical 2-inch section of the loop between FiberStrike sensors 1 and 2. To collect variable datasets data one leak was opened at a time to see if there would be a difference in the signature between the two. To simulate a blockage in the pipeline a control valve on the main path was closed partial way and then reopened while recording data from all four sensors. In another test, a bypass valve was opened all the way that allows for flow to be redirected into the reservoir tank through a shorter path while keeping the main overhead path fully open. To simulate physical impacts the pipeline was struck in different locations using hammers, wrenches, and other blunt objects. Finally, data recordings were made while there was no activity on the loop and then while the pump turned on and established a stabilized flow through the loop.

\subsubsection{Feature Extraction Algorithms}

While the raw analog signal from the acoustic transducers can be viewed directly for anomalies caused by leaks or other events, there is often so much noise that nuances in signal behavior are overlooked. In addition, when comparing multiple signals, it is necessary to manipulate the data to gain the most insights.

\section{Fast Fourier Transform}

The discrete Fourier Transform (DFT) converts a sample set of data in the time domain to the frequency domain. The DFT is achieved by separating the amplitudes of a signal based on its frequencies [66]. When graphed as in Figure 3.9, the result is the amount of energy present in each frequency domain of a signal. 

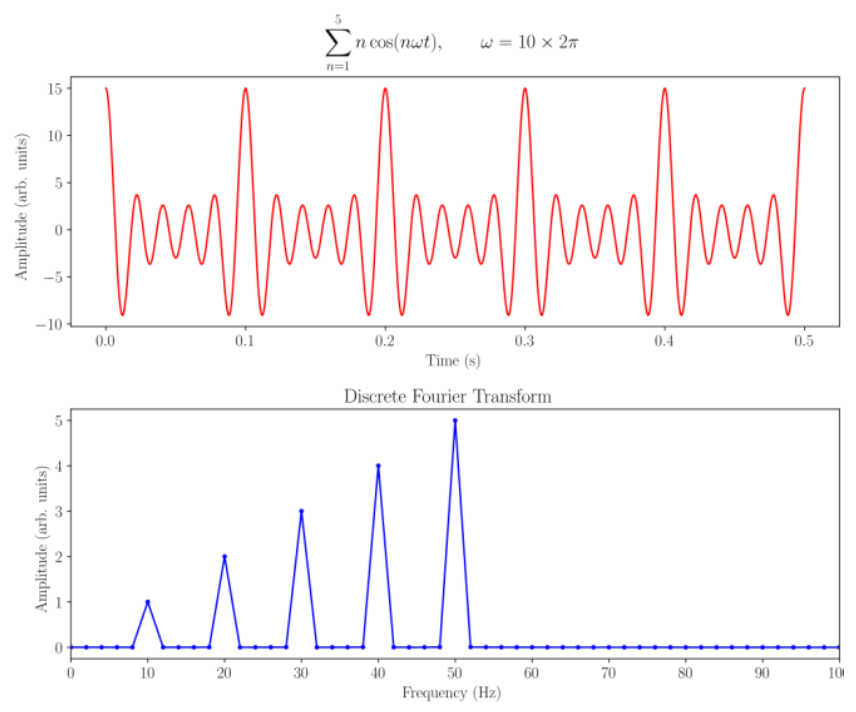

Figure 3.9 - Fourier Transform of a Cosine Wave

The fast Fourier Transform (FFT) is a method to calculate the DFT of a signal or sequence in a more computationally efficient manner. The time complexity of the DFT is $O\left(N^{2}\right)$ compared to $O(N \log (N))$ of the FFT [67], making it much more suitable for digital signal processing.

The FFT is useful for analyzing the frequency spectrum or power spectral density (PSD) of the acoustic signals recorded on the pipeline. Harmonic signals from rotating machines such as pumps will show up at as peaks at specific frequencies whereas leak signals can be modeled as a random signals [68]. Applying FFT to a recording of normal operation of the pipeline versus a recording when a leak is present will show an increase in amplitudes in random frequencies across the spectrum. For the purposes of this work, the FFT was applied using the Python NumPy library fft.fft() function [69]. 


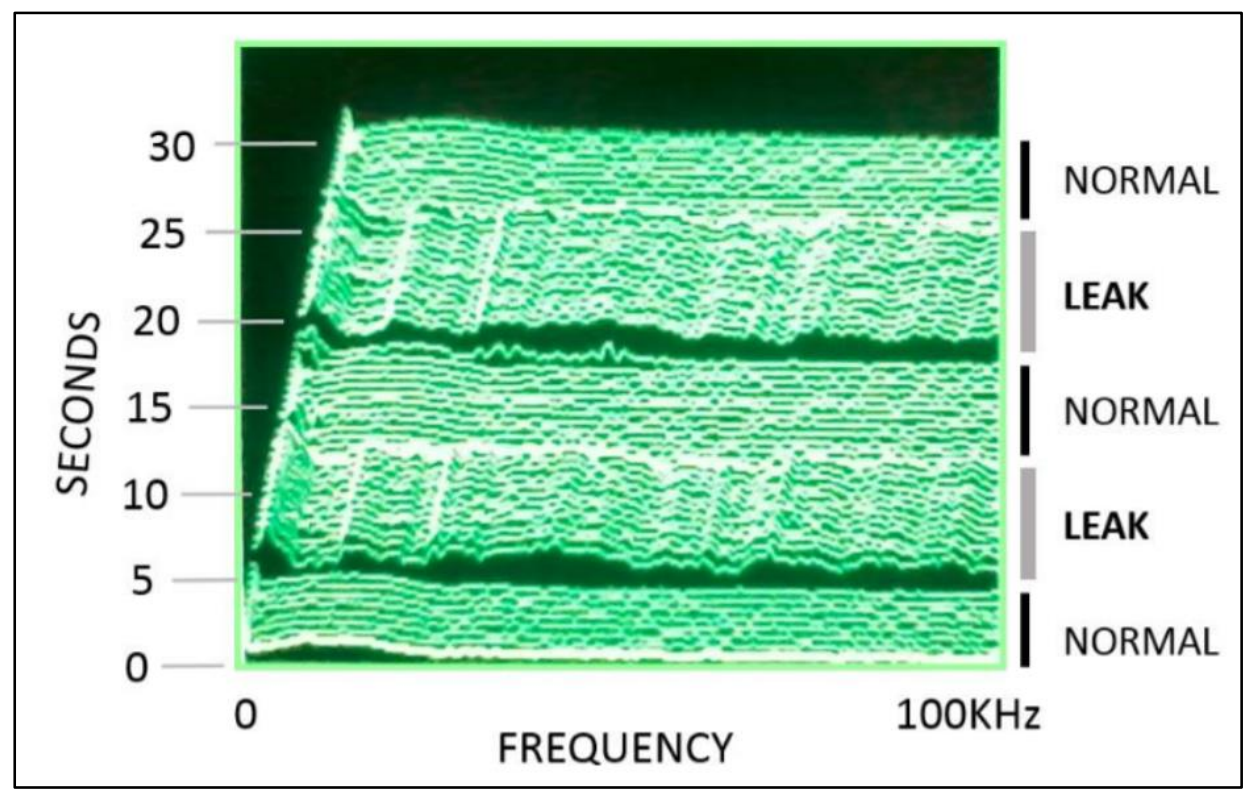

Figure 3.10 - Change in Power Spectral Density over Time [55]

By applying the FFT to windows of a signal and combining the data together, a time-lapse of the power spectral density of the pipeline can be achieved as shown in Figure 3.10, a graph provided by CEL. During normal operation, the PSD should remain relatively stable, however, the occurrence of a leak or other malfunction on the pipeline will show up as a destabilization of amplitudes across various frequencies.

\section{Cross-Correlation Method}

The relative location of a leak can be found using the cross-correlation method by first finding the time lag $\tau_{\max }$ between signals from two acoustic sensors spread apart on a pipeline. The cross-correlation function is used to measure the similarity of two sets of data and returns a coefficient between -1 and 1; 1 meaning a perfect correlation, 0 meaning no correlation, and -1 meaning a negative relationship between the two signals [70]. When a signal is recorded at two locations varying distances from the origin, the cross-correlation 
function will result in a low correlation coefficient because initially the signals are out of sync as represented in Figure 3.11.
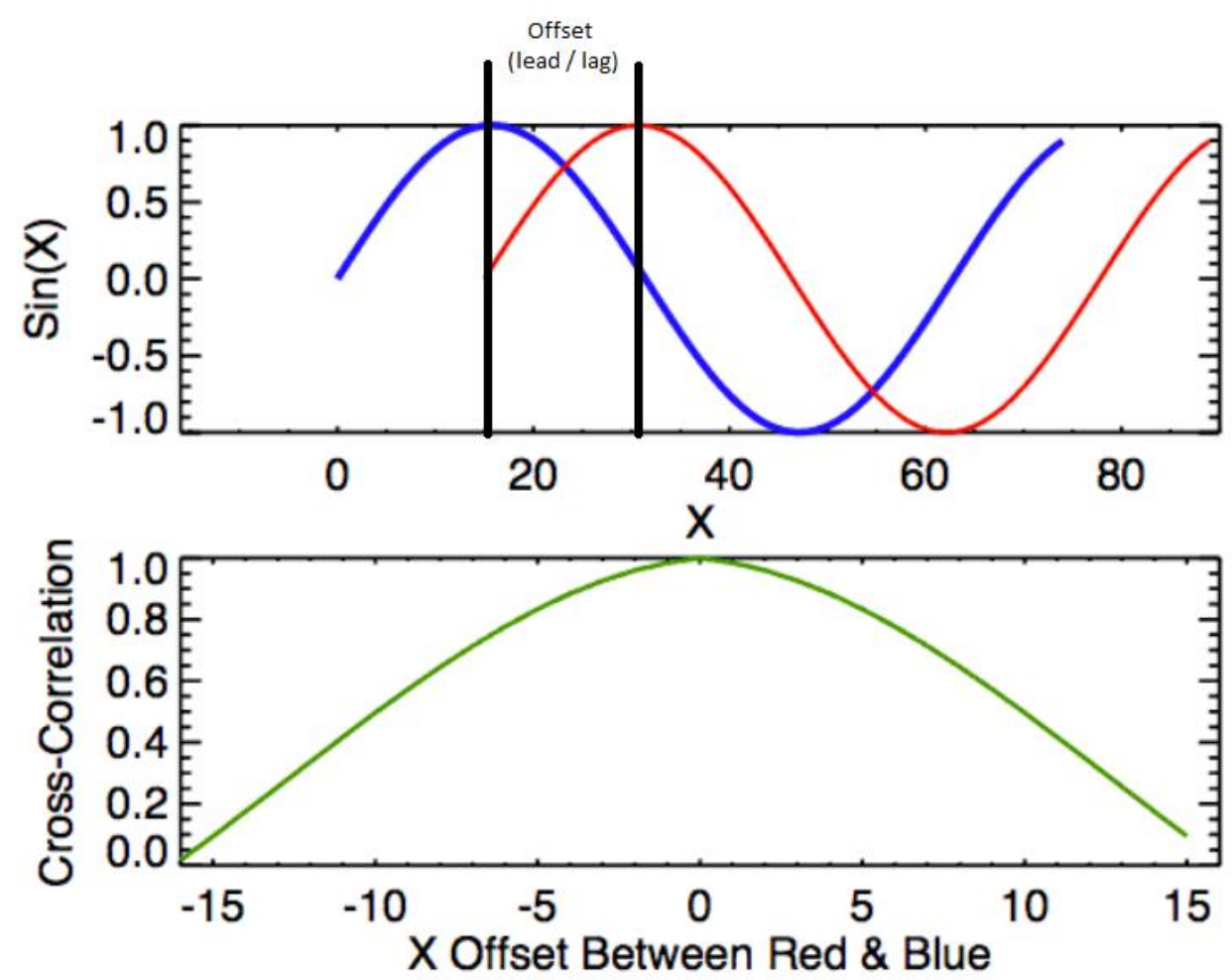

Figure 3.11 - Calculating time lag of a signal with the cross correlation function [71]

By increasing the phase shift of one signal versus the other and calculating correlation coefficient at each interval, the resulting continuous function will have an absolute maximum which represents the time lag between the two signals as shown in Figure 3.12.

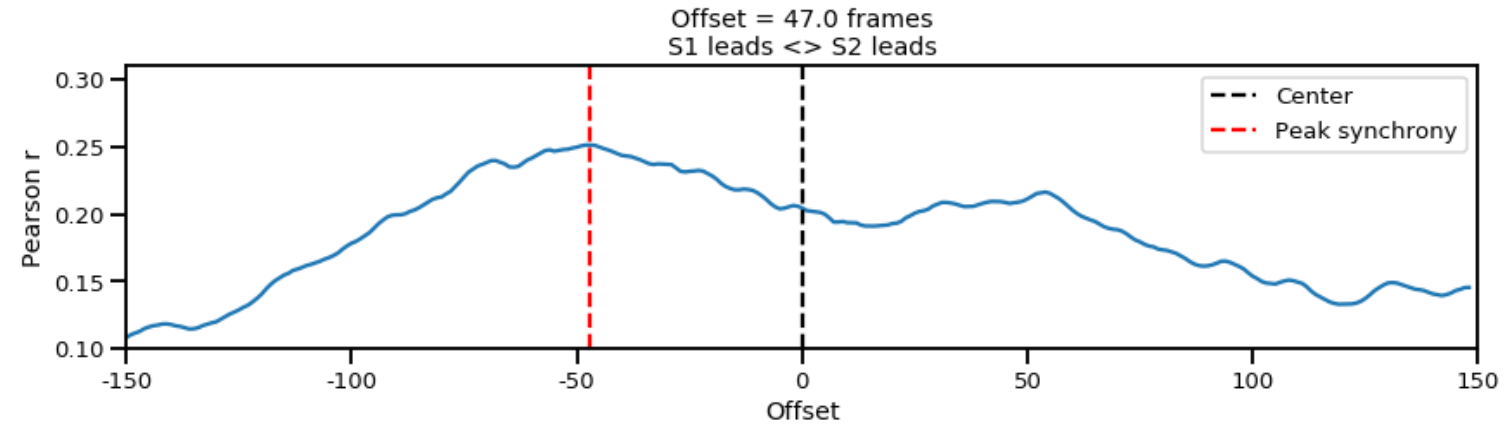

Figure 3.12 - Correlation coefficient as function of signal offset [72] 
The offset is the number of samples or data points that the shifted signal is moved until peak synchrony is achieved. The time lead or lag of the signal is then simply calculated by multiplying the offset by the time interval between each measurement.

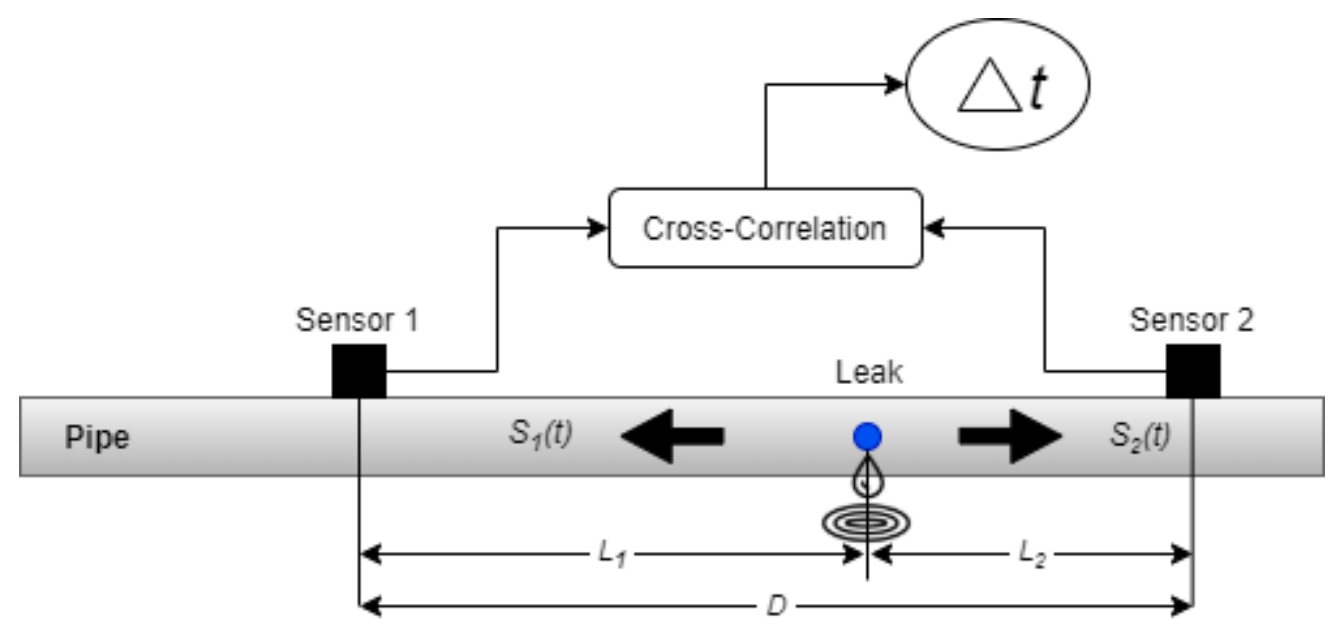

Figure 3.13 - Applying the Cross-Correlation Method to Find the Leak in a Pipe [46]

Figure 3.13 provides a visual representation of how the distance to the leak is then determined after finding the time delay $\Delta \mathrm{t}$ between the two signals. Then using the equations below to find the distance to the leak [73]:

$$
L_{1}=\frac{D+c \Delta \mathrm{t}}{2}, \quad L_{2}=D-L_{1}
$$

Equation 1 - Relative distance from sensors to leak

The distance to the leak relative to sensor 1 and sensor 2 are denoted as $\mathbf{L}_{\mathbf{1}}$ and $\mathbf{L}_{\mathbf{2}} ; \mathbf{c}$ represents the velocity of sound propagating through the pipe; $\mathbf{D}$ is measured as the length of pipe between sensor 1 and sensor 2. It should be noted that propagation velocity needs to be determined experimentally or calculated based on pipe material and dimensions. 


\subsection{Results and Discussion}

To make any conclusions about the deviation of performance of a pipe system from normal operation, the baseline acoustic signal must first be captured. The raw acoustic signals of all channels are shown in Figure 3.14. Due to the proximity of the pump, there is a lot of noise that exhibits harmonic tendencies. It can easily be seen that the signal from channel 1 (red) has a noticeably lower amplitude compared to the other signals, this can be explained by its proximity to a brace supporting the pipe loop, dampening the vibrations. The other sensors are mounted higher and are subject to more movement. Regardless of amplitude, it can be seen that each channel maintains relatively consistent behavior.

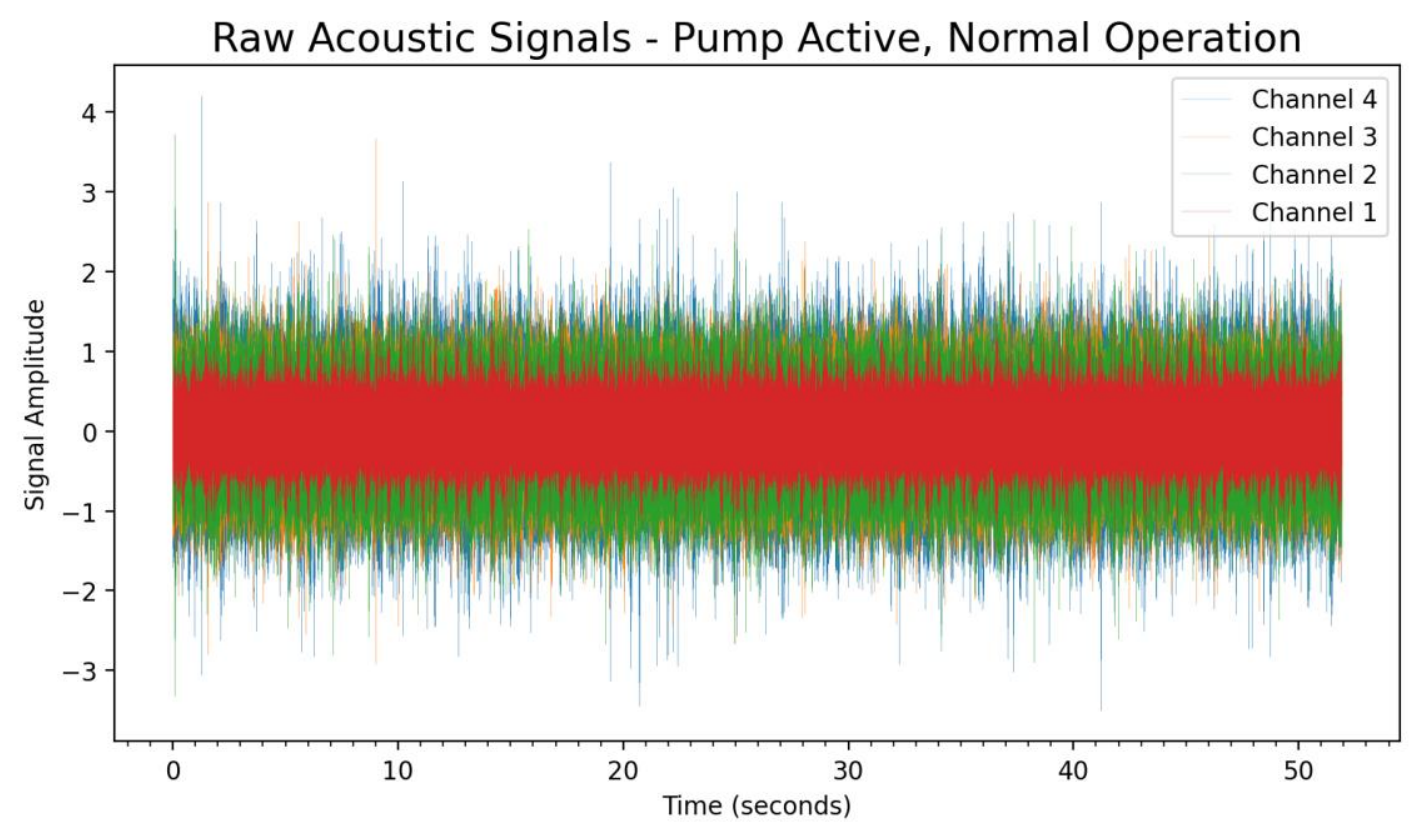

Figure 3.14 - Raw acoustic data from all sensors during normal operation

The raw signal is hard to extrapolate finer details from as the noise can obscure signal behavior in the frequency domain. The next step is to analyze the frequency spectrum and how energy levels in individual frequencies change over time. 


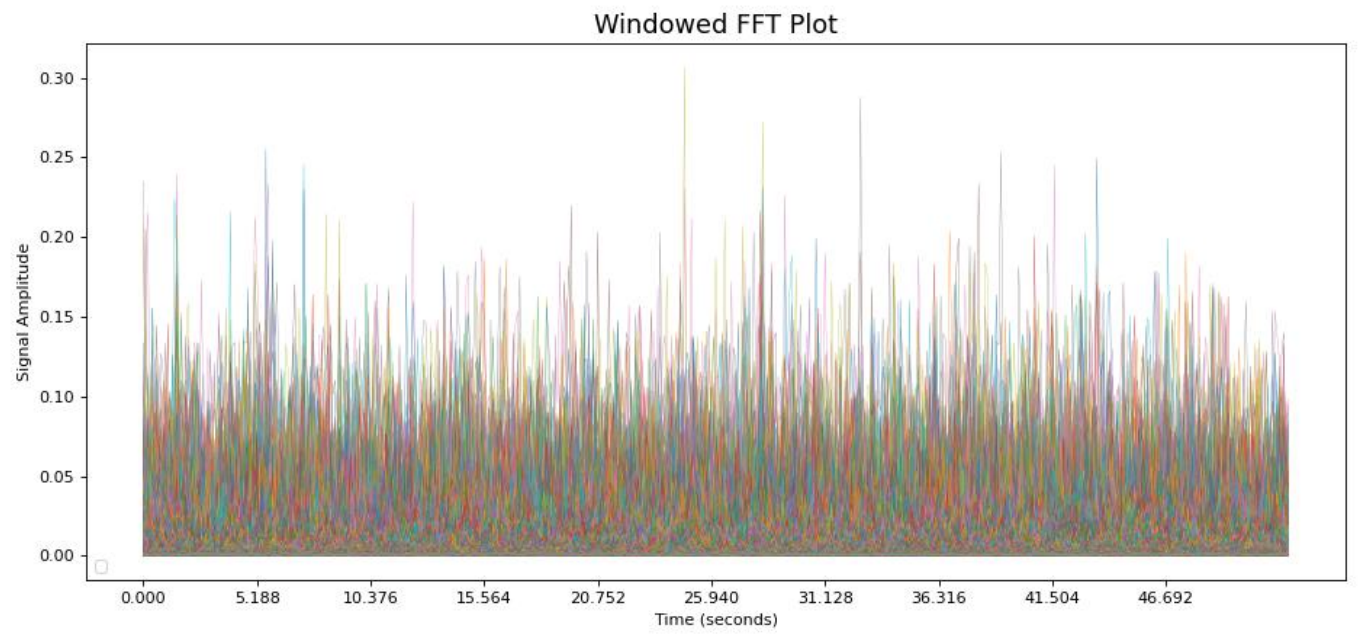

Figure 3.15 - Windowed FFT plot of normal pipeline operation

The raw signal in segmented into sets of 5000 data points (approximately $100 \mathrm{~ms}$ ); FFT is applied to each set resulting in 2500 values representing vibrational energy present in different frequency components between $1-25 \mathrm{KHz}$. Each set of 2500 points is then plotted, each frequency represented by a time series as seen in Figure 3.15. Due to the high density of series extrapolation of information again becomes difficult.

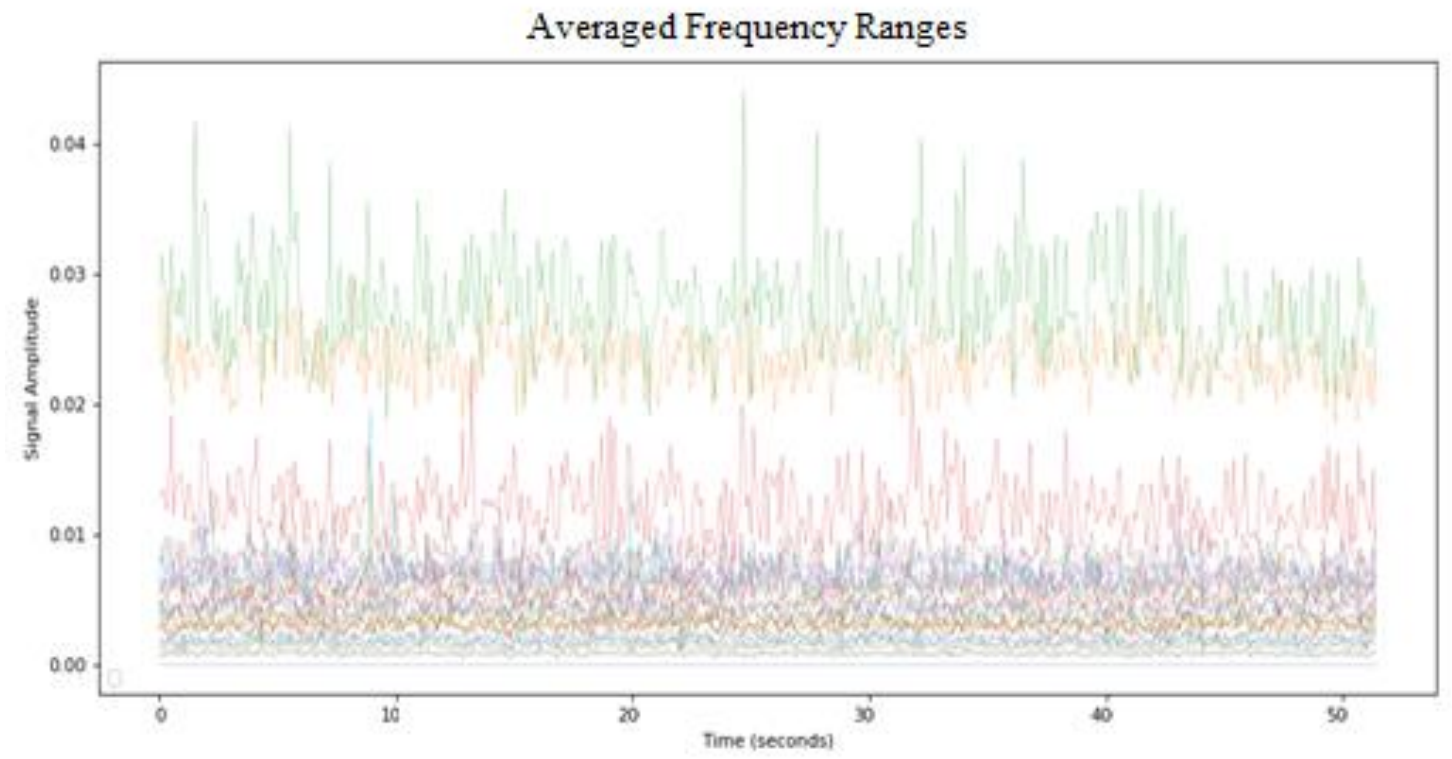

Figure 3.16 - Windowed FFT (averaged frequencies) plot of normal pipeline operation 
By again segmenting the 2500 data points per FFT set and taking the average of 25 equal and contiguous sets, a more effective plot of the change in frequencies can be achieved as shown in Figure 3.16. Once the baseline was achieved it different representations, data collected during faults can be compared.

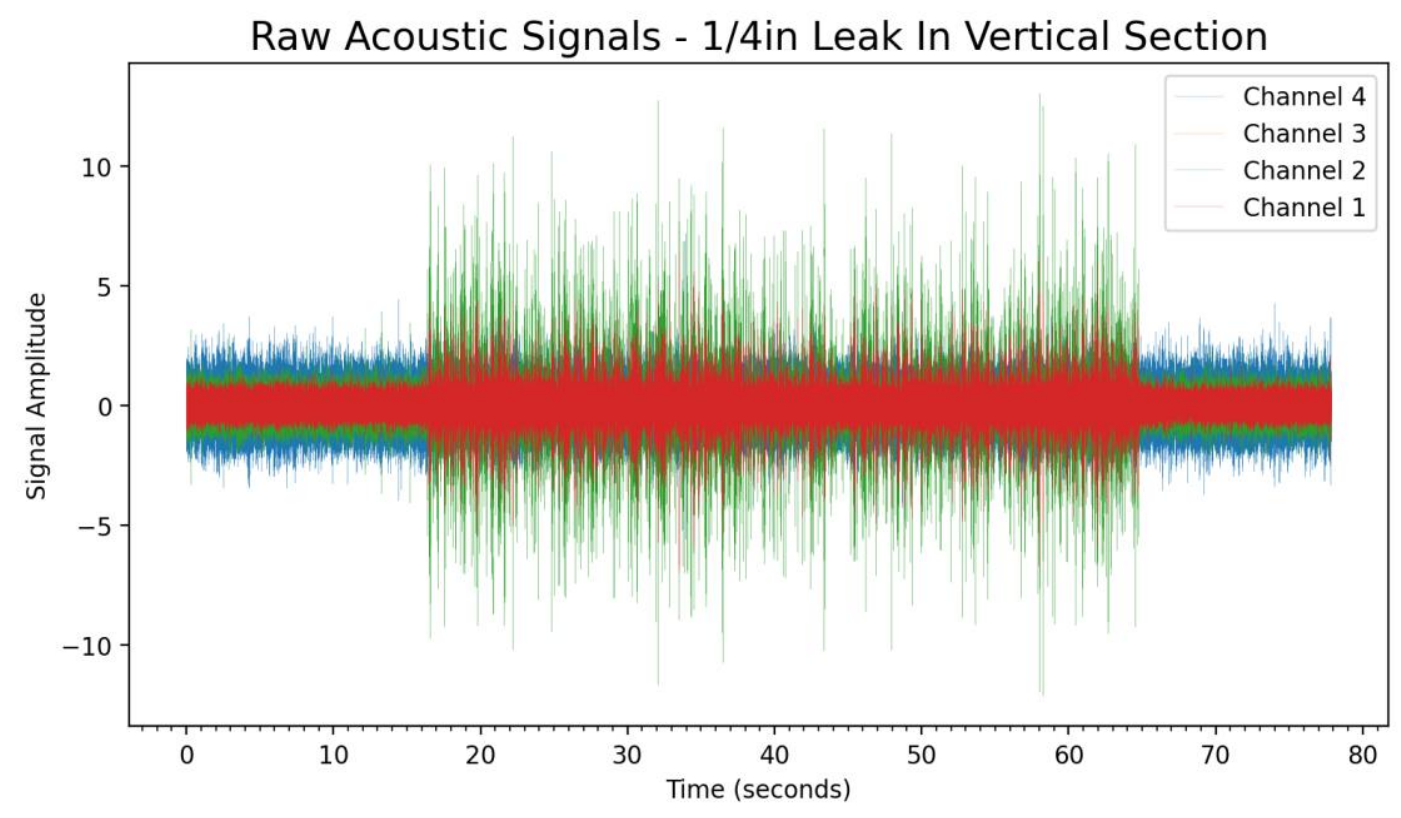

Figure 3.17 - Acoustic sensor data while 1/4" hole leak

A $1 / 4 "$ " hole was opened, allowed to leak for about a minute, and then sealed again while recording signals from the acoustic sensors. In Figure 3.17 the raw signals show an immediate response, however, the further the sensor from the location of the leak, the less the leak signal stands out from the noise. When the windowed FFT method with averaged frequencies is graphed in Figure 3.18 it can be seen that the leak signal mostly causes disturbances in frequencies between $1-2 \mathrm{KHz}$ (green) and $2-3 \mathrm{KHz}$ (yellow), but that the majority of frequency series do not significantly different behavior from the baseline. 


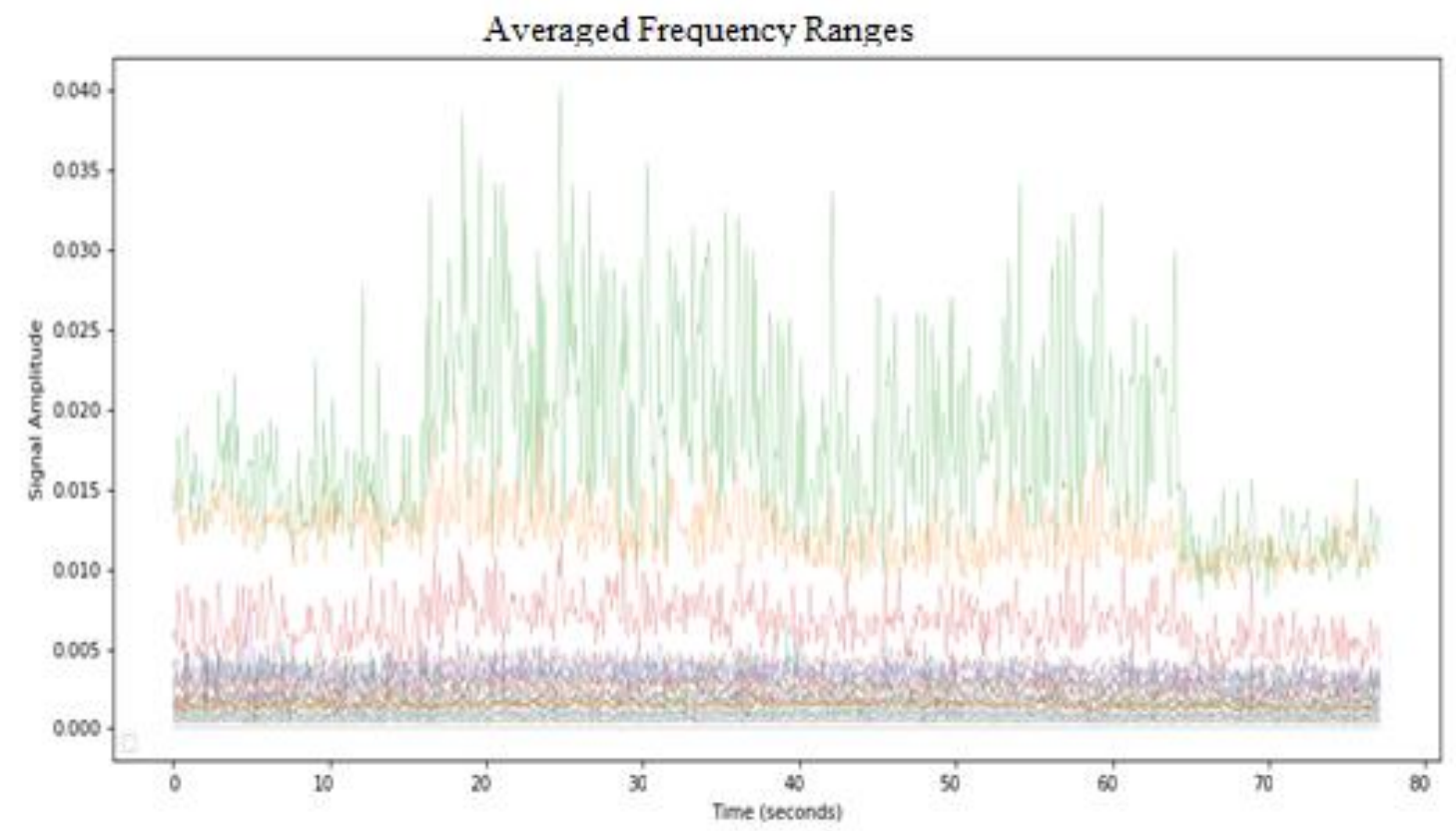

Figure 3.18 - Windowed FFT (averaged frequencies) plot of leak

Once the presence of a leak was confirmed determining the relative location to the sensors was attempted through the cross-correlation method. Despite the high level of noise, it was still possible to find a high correlation coefficient between the leak signals recorded by two different sensors.

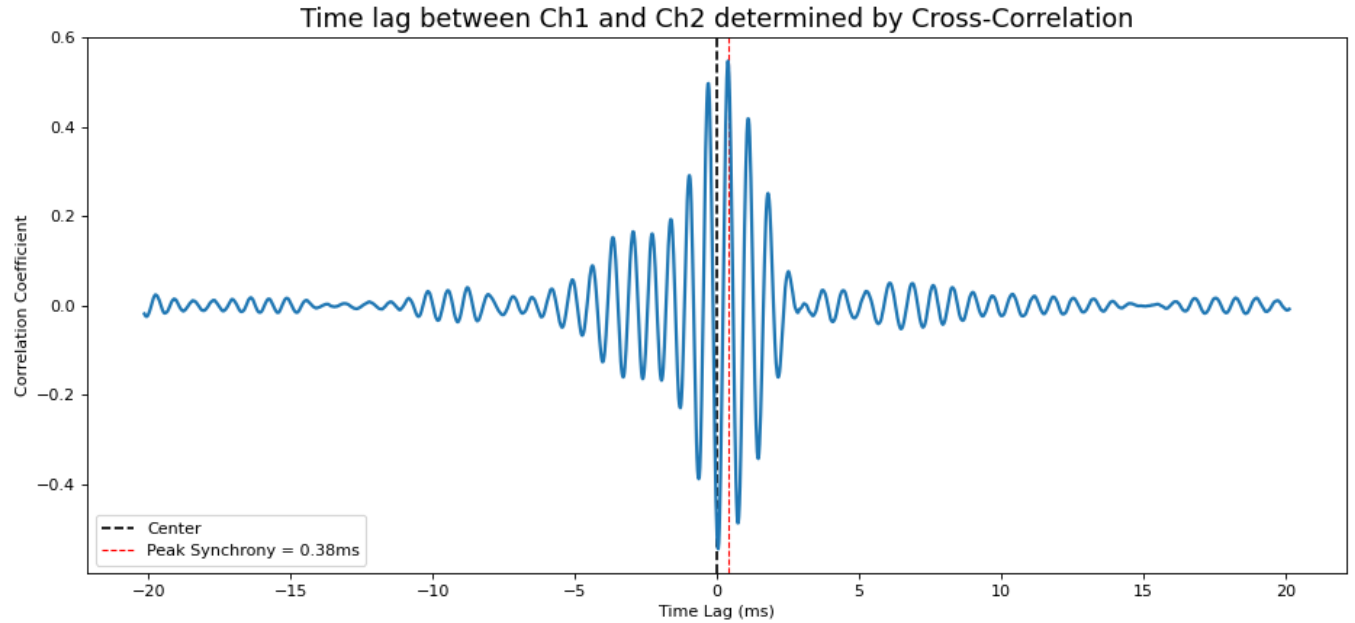

Figure 3.19 - Time offset of leak signal by cross-correlation $\left(t_{0}=20.0 s, t_{1}=20.1 \mathrm{~s}\right)$ 
The leak signal from the channel 1 sensor was used as the basis to compare the shifted signal and peak synchronicity was found to be at +0.38 ms indicating that the channel 2 signal leads the channel 1 signal, meaning the leak is closest to the channel 2 sensor. The relative distance can then be found by applying the equations discussed in Feature Extraction Algorithms. To verify the time lag the cross correlation method was applied to a different segment of the signal 20 seconds apart and had agreeable results as shown in Figure 3.20 which yielded an offset of $+0.40 \mathrm{~ms}$.

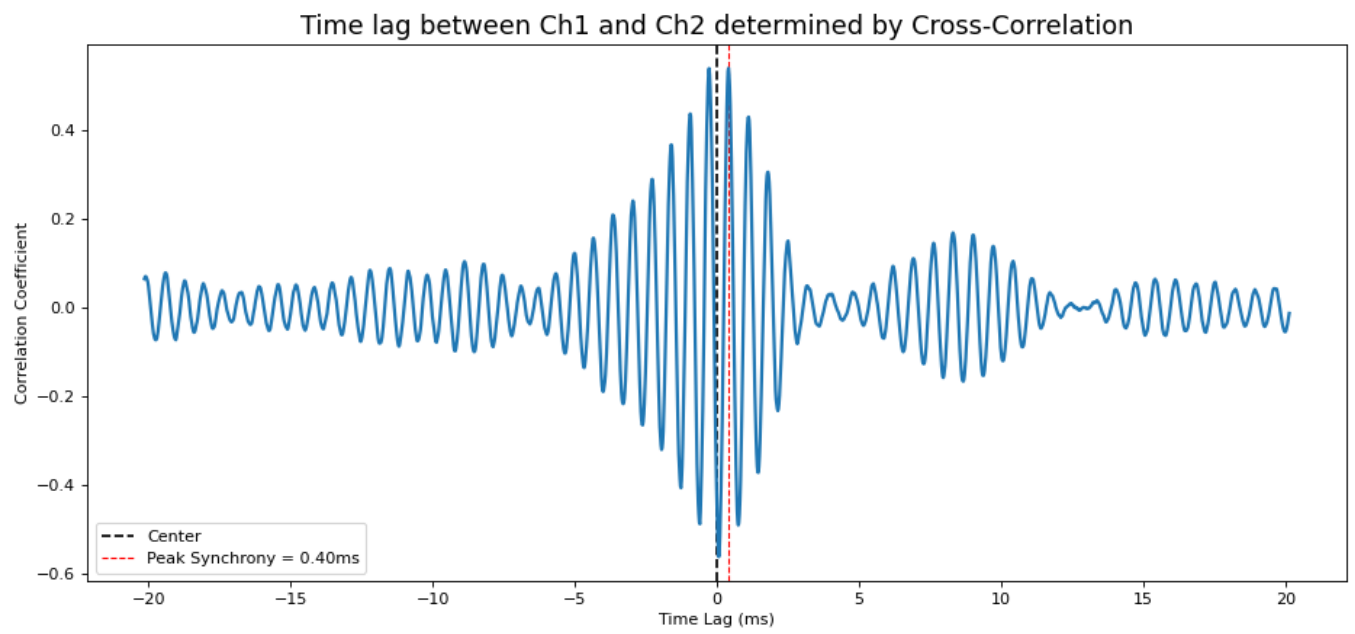

Figure 3.20 - Time offset of leak signal by cross-correlation $\left(t_{0}=40.0 \mathrm{~s}, t_{1}=40.1 \mathrm{~s}\right)$

The locations of sensor 1 and 2 on the pipeline are approximately 1 meter apart with $1 / 4$ " hole being approximately 0.3 meters from sensor $2,0.7$ meters from sensor 1 . Significant time was not spent on calculating the propagation velocity of sound through the pipe loop as it has thinned walls, fittings, and other geometries that would require an empirical approach to finding the true value. Instead, the velocity for sound propagation through water at room temperature was used as it would represent the closest known value. The velocity for sound in water at room temperature (25C) used for these calculations was: 


$$
c=1,496 \mathrm{~m} / \mathrm{s}[74]
$$

Taking an average of the two reported time lags:

$$
\Delta \mathrm{t}=\frac{0.38 \mathrm{~ms}+0.40 \mathrm{~ms}}{2}=0.39 \mathrm{~ms}
$$

Previously it was stated that the sensors were 1 meter apart, therefore:

$$
D=1 m
$$

And finally, by inserting these values into Equation 1 we get:

$$
\begin{gathered}
L_{1}=\frac{1+(1496)(.00039)}{2}=0.79172 \\
L_{2}=1-0.79172=.20828
\end{gathered}
$$

While admittedly the propagation of sound through a water filled schedule 802 " pipe was not determined with scientific rigor, simple back of the envelope calculations provide evidence that the acoustic signals on which the cross correlation method was applied resulted in an accuracy of leak localization of \pm 0.1 meters. The measured distance from sensor 1 to the leak was 0.7 meters instead of the calculated 0.79 meters, an overshoot, and the distance to sensor 2 was measured at 0.3 meters instead of the calculated 0.208 meters. Further, the propagation of sound through water filled steel pipe can be expected to be greater than simply the velocity of sound through water, which was used in these calculations, this would further increase the error. A more rigorous approach to testing the cross-correlation method with the CEL FiberStrike system is needed to draw valid conclusions, however, these initial results are promising.

To test the sensitivity of the leak detection system a smaller hole of $1 / 8$ " was opened between sensors 1 and 2 and left to leak while recording the acoustic signals from the pipe loop. The leak signal was not as prominent as the larger $1 / 4$ " hole, however, the presence 
of aberrant behavior in the acoustic signature can be seen in Figure 3.21. The hole is opened at approximately 35 seconds elapsed and left to leak for the duration of the recording. Fluctuations are primarily seen in channel 1 and instead of the large increase in signal amplitude seen in Figure 3.17, the leak signal can be characterized as a decrease in amplitudes. This coincides with observations at the time of data collection that witnessed the hole in the pipe expelling water and then pulling in air in a harmonic rhythm. The decrease in amplitudes of the signal may have been caused by the decrease in pressure in the pipe when air was let in near the channel 1 sensor. When the larger $1 / 4$ " hole was opened, a constant stream of water was present and did not exhibit any noticeable harmonic pressure changes.

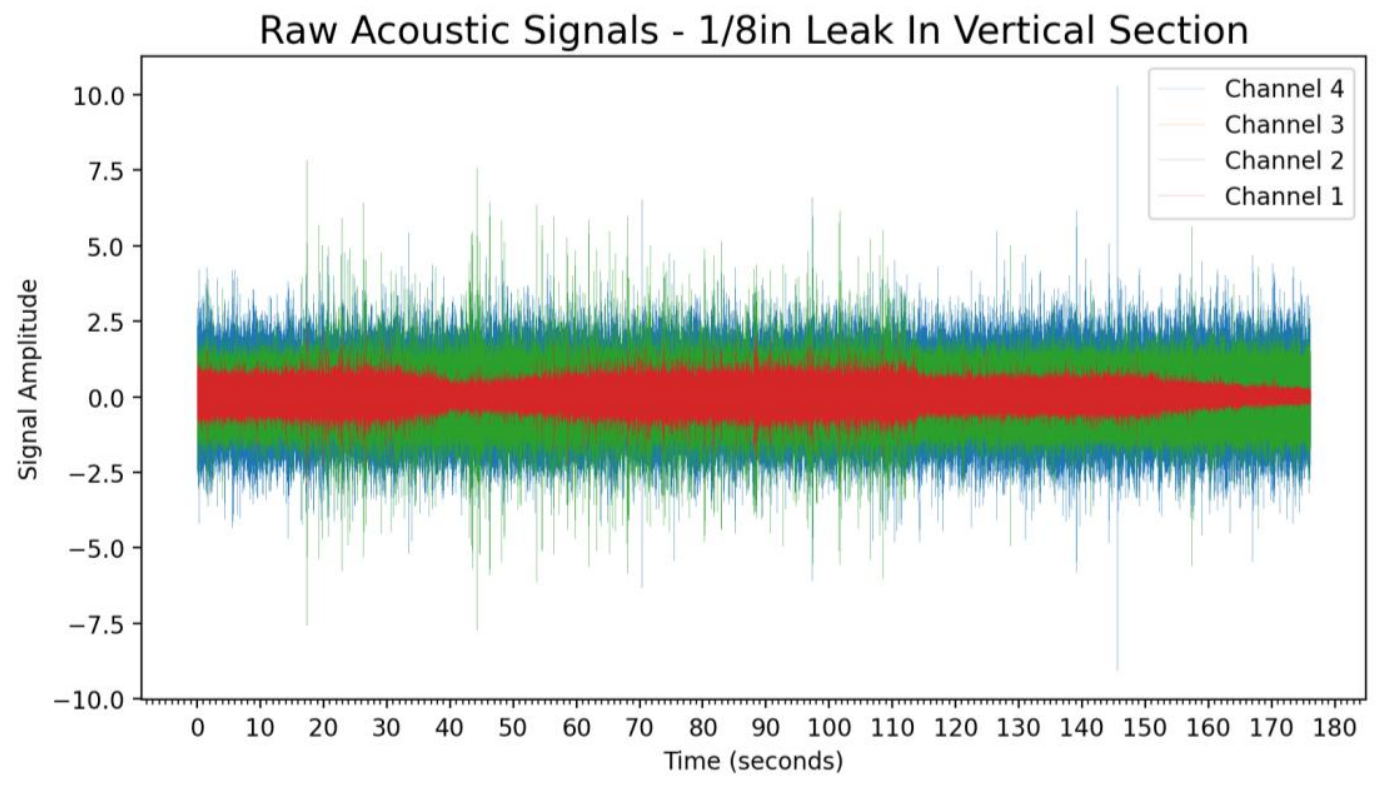

Figure 3.21 - Acoustic sensor data while 1/8" hole leak

After applying the windowed FFT method a deviation from normal behavior can be seen again, however, it can be characterized by a significant decreased in energy in the $1-2 \mathrm{KHz}$ frequency range (green) and an overall decrease in the amplitudes of all frequencies. 


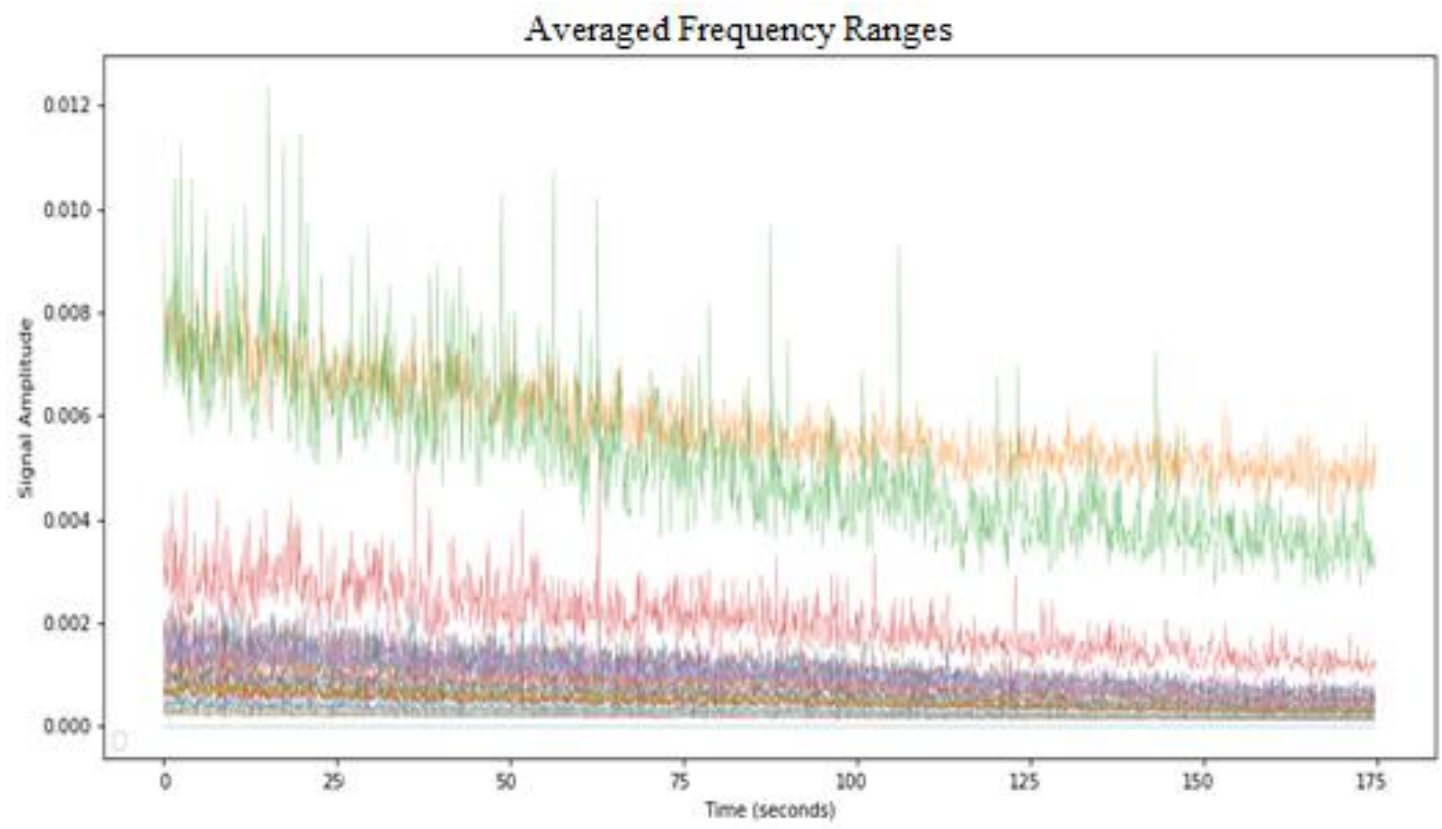

Figure 3.22 - Windowed FFT (averaged frequencies) plot of 1/8" hole leak

The signatures of other events were recorded as well and analyzed to determine the method by which the acoustic signature of the pipeline was affected. A pipeline monitoring system should not only be able to detect structural damage such as leaks or physical impacts, but also deliberate actions such as the opening and closing of passages. Valves for stopping or redirecting flow are ubiquitous on pipelines and the more complex the more automated the control systems for these valves can become. Should software malfunction, a worker turns the wrong valve, or a valve fail to open all the way, it is important for a monitoring system to be able to detect these events. To collect data, a bypass valve was open fully that allowed for a shorted return path from the pump to the reservoir tank. The valve to the overhead section of pipe remained open and a small amount of flow was still present while the majority redirected. 


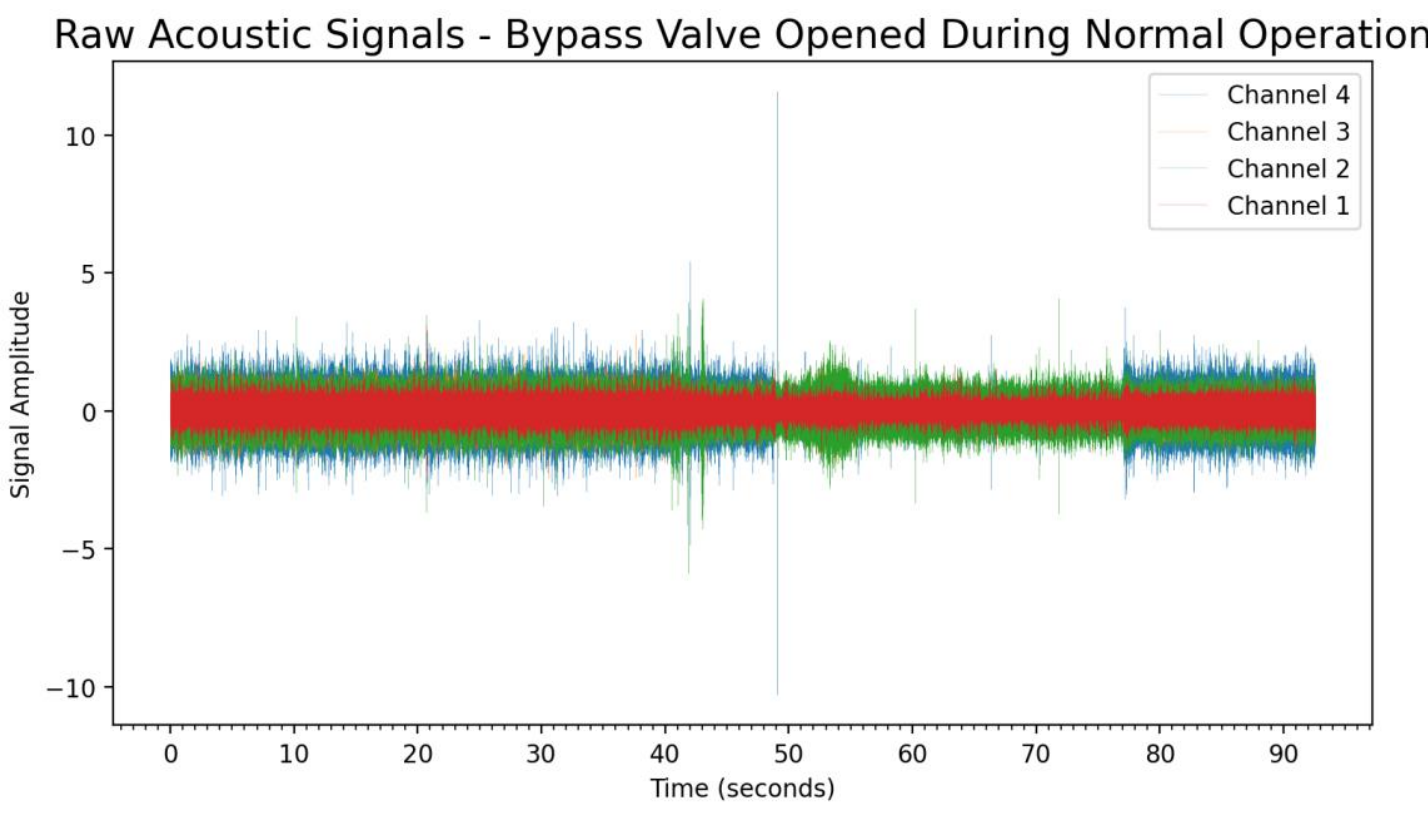

Figure 3.23 - Acoustic sensor data while bypass valve opened

The valve was slowly opened starting at approximately 45 seconds and then closed again after approximately 30 seconds.

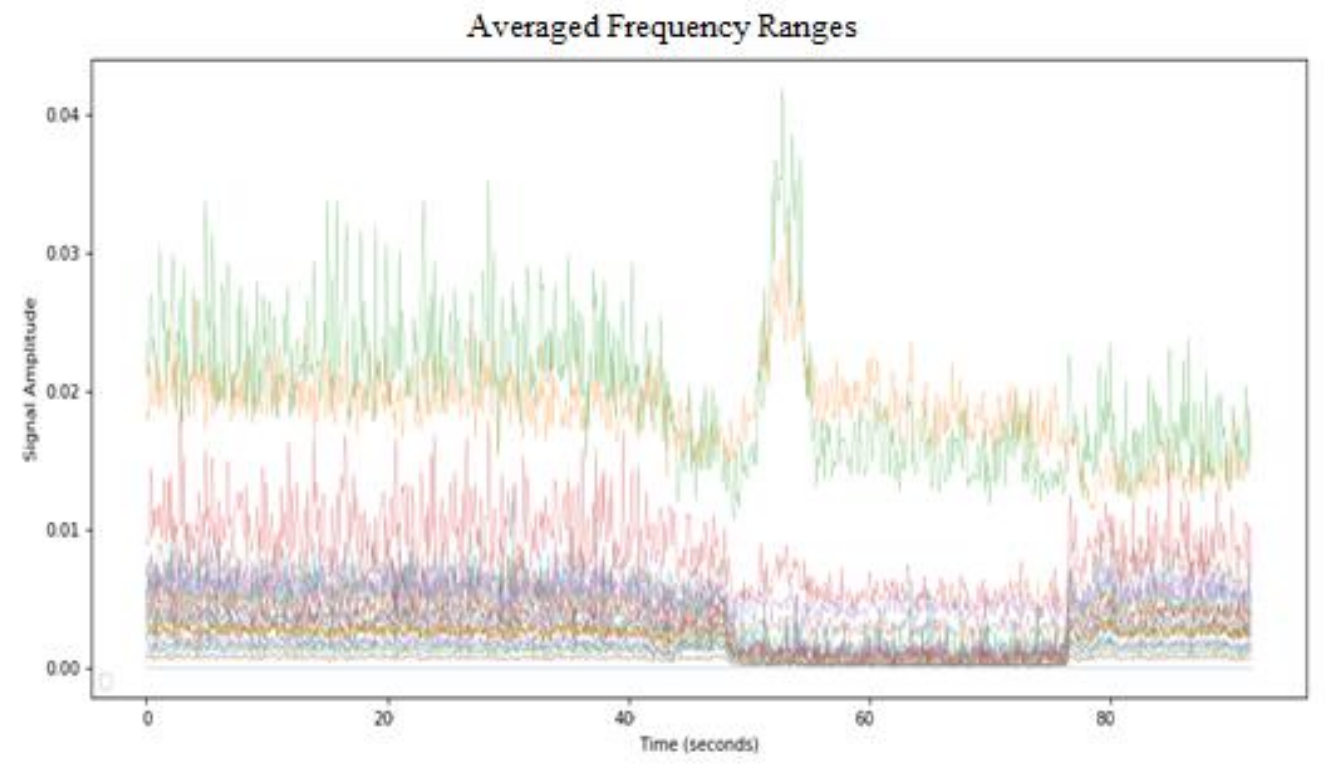

Figure 3.24 - Windowed FFT (averaged frequencies) plot of bypass valve opened 
In Figure 3.23 it can be seen that the signals from channels 3 and 4 dissipate significantly and channels 1 and 2 dissipate only slightly due to their close proximity to the higher volume of flow. Analysis of the changes in frequencies provide an even clearer picture of the event as Figure 3.24 shows a sudden and significant drop in amplitudes of a certain range of frequencies while a spike occurs in the $1-3 \mathrm{KHz}$ range. This could be a result of the vibrational noise caused by opening valve and the sudden change in pressure as the flow redirected, causing a large vibration along the pipe loop.

To simulate a blockage, another test partially closed the main valve controlling flow to the overhead section of pipe. The bypass valve was closed for this test and all other parameters remained the same as normal operation except that the main valve was turned to approximately $3 / 4$ open after 12 seconds and then opened fully again at the 35 seconds mark.

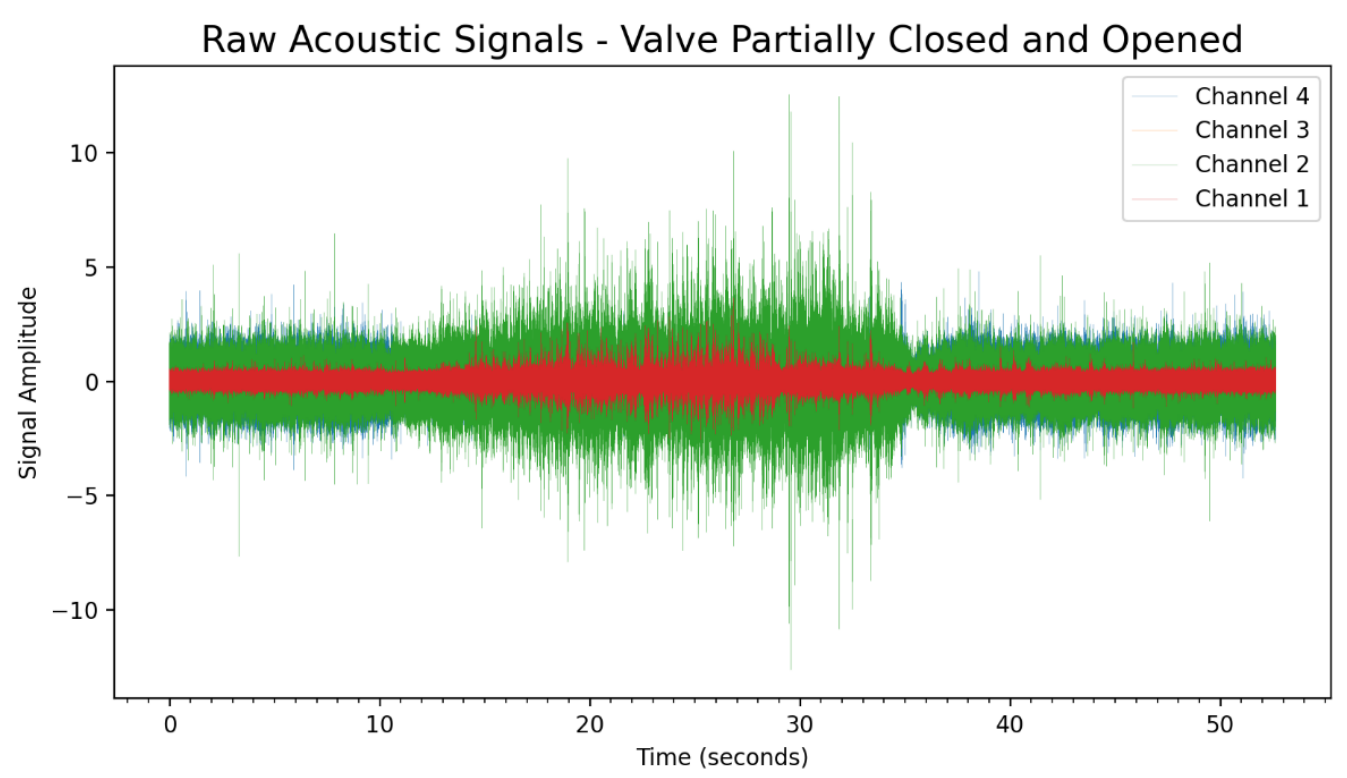

Figure 3.25 - Acoustic sensor while during main valve partially closed 
The change or pressure and fluid velocity resulted in more noise and randomness in the acoustic signal of the pipeline. The valve being just before channels 1 had the greatest effect on the signals from channel 1 and 2, but still affected the signals from channels 3 and 4 , most noticeably when the valve was turn.

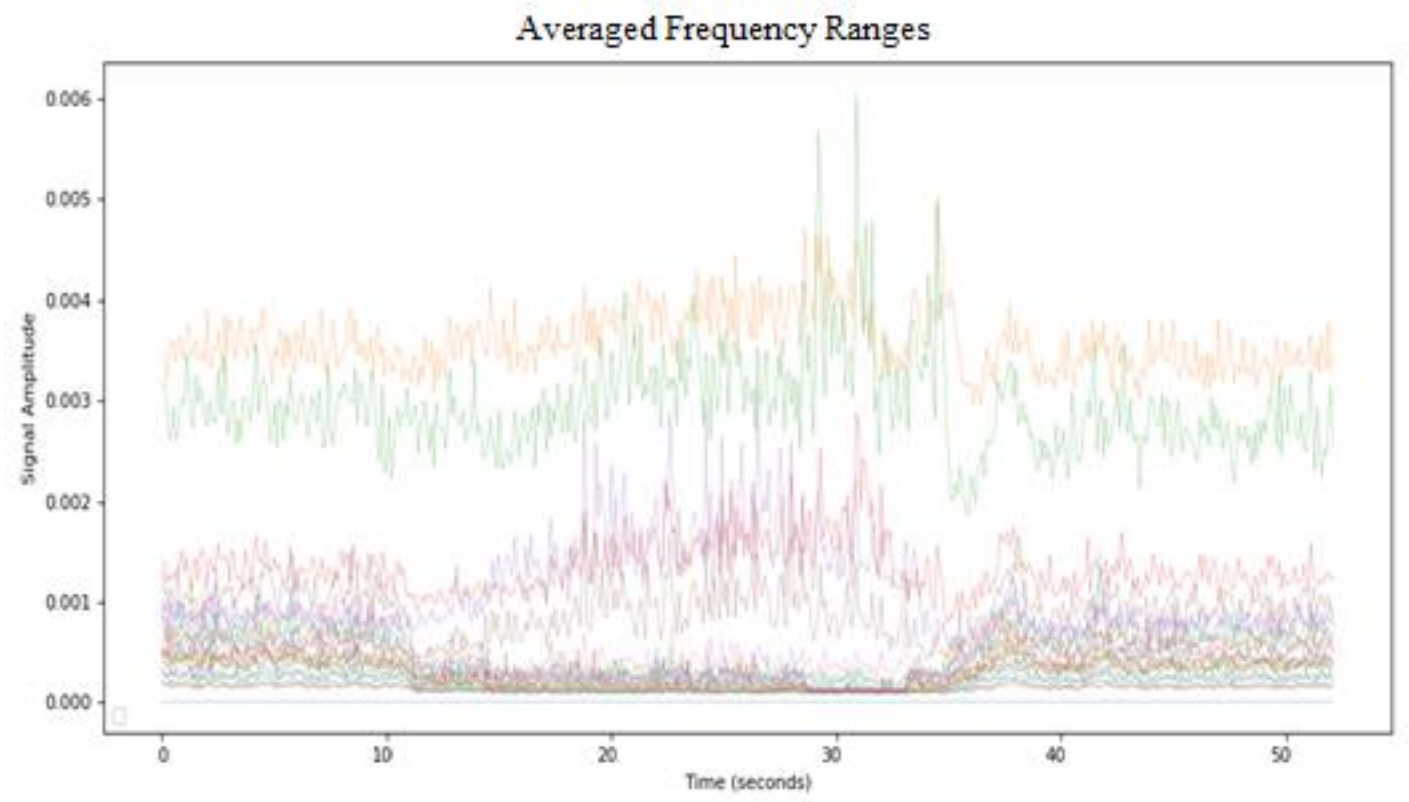

Figure 3.26 - Windowed FFT (averaged frequencies) plot of main valve closure

The local increase in pressure before the valve and increased fluid velocity passing through it resulted in a significant increase in the amount of energy in some frequencies and while others exhibited extremely low energy levels as seen in Figure 3.24 when the bypass valve was opened.

Finally, a constant threat to any pipe is damage caused by physical impacts. Buried pipes are always at risk of being struck during excavation projects and in the oil and gas industry vandalism or siphoning occurs on a regular basis. To capture the signature of an impact the pipeline was struck with a hammer in various locations and exhibited a unique signature as seen in Figure 3.27. A massive spike in amplitude across all sensors was 
witnessed regardless of the location of the strike. The further away from the strike, the amplitude of the signal was lower but still far above the normal operating threshold.

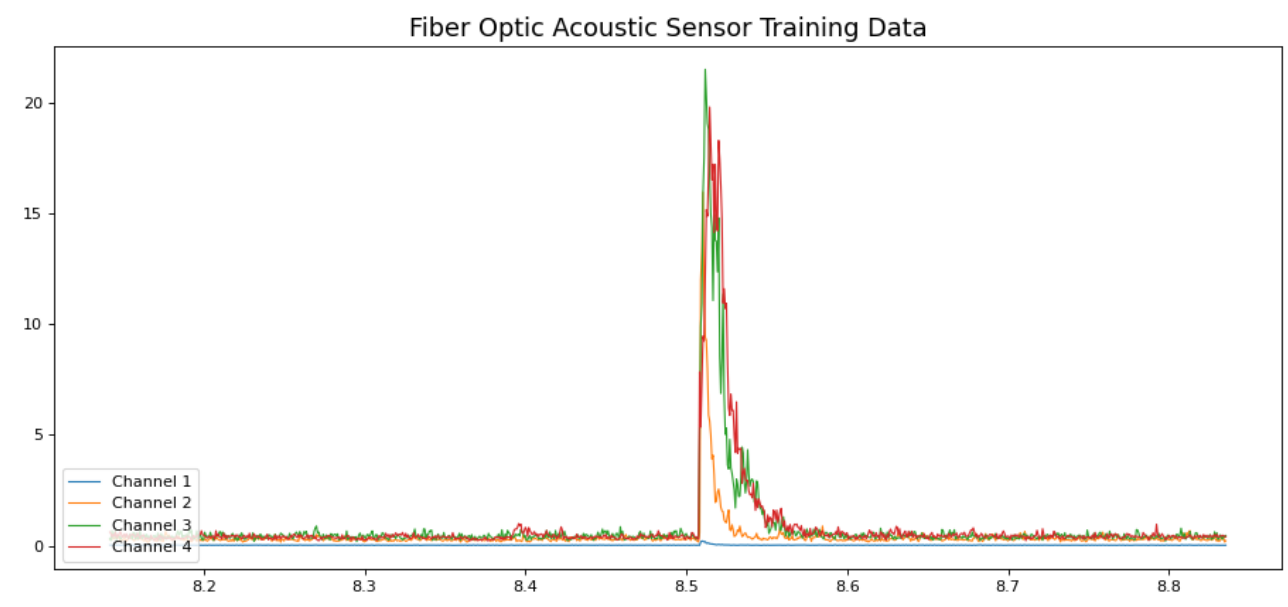

Figure 3.27 - Raw acoustic data from all sensors during hammer strike

Due to the uniqueness of the signature and the high signal to noise ratio, it was also possible to apply the cross-correlation method to determine the location of the impact. In systems where there is a high level of noise caused by machinery, the increased number of sensors sampling the acoustic signal of the pipe line allows for a higher degree of certainty of any given event and also increases the accuracy when applying the cross-correlation method.

\subsection{Conclusion}

This work has attempted to investigate the functionality and application of acoustic transducers interpreted by optical interferometry, and the possible application in nuclear waste facilities where pipelines must be monitored. The CEL Fiberstrike system was assessed for its ability to detect unique events such as impacts and leaks on a carbon steel pipe loop located near an active pump. This sensor system was shown to register numerous types of pipeline events in real-time with little to no visible delay. By using 2in and 3in 
pipe sections, the bench scale testing documented in this work provides basis for the sensor system's potential implementation in various DOE sites when transferring hazardous materials through a pipeline that requires real-time monitoring. This will ensure that any fault is detected immediately and reduce the impact. While all analysis of captured data was done by researchers, it can easily be seen that through training a machine learning model with various types of pipeline fault signatures, an automated real-time monitoring system could be realized. Further investigations into augmenting the feature extraction methods and training a model on the results shown here is planned.

\section{Machine Learning Model for Pipeline Fault Detection}

\subsection{Introduction}

Infrastructure monitoring is a vital part of reducing maintenance costs and ensuring safety. As regulations in the nuclear industry continue to tighten effective solutions that do not rely directly on humans to monitor systems is required to reduce error and process higher volumes of data. Machine learning models (MLM) are algorithms that are capable of improving the accuracy of an output as the amount of data passed through the algorithm increases. By storing augmentations in the form of weights that are adjusted each time the model is trained, it is possible to several different types of algorithms that can discriminate between different sets of data of the same format without any knowledge of the features or attributes it is using to identify the differences between the data sets [75]. This makes machine learning models extremely attractive for discriminating phenomenon that are understood best empirically and require processing large amounts of quantitative data. Therefore, identifying changes in an acoustic signature make for an ideal problem to apply 
machine learning due to the large amount of data and large number of unknown variables that affect the change in data.

Starting with a long short-term memory (LSTM) neural network, an anomaly detection model was developed to analyze the data generated by the CEL Fiberstrike system, fiber optic acoustic sensors mounted on a piping system. The sensors detect vibrations in the pipes through attenuation of spooled optical fiber inside of them, the light signals are then converted into digital values in an optical interrogator data stream which then outputs the data in real-time over ethernet via User Datagram Protocol (UDP) to a remote host so the anomaly detection model can be run on any external machine. The model was developed in Python, making use of Keras, Tensor Flow, NumPy, and Pandas libraries. Once preprocessed, the datasets for nominal operation were used for training of the LSTM model. Once trained on normal operating conditions, the model was asked to predict the behavior of new sets of data that included the acoustic signatures of events and faults such as leaks, impacts, or valve operation. The deviation from the model's predictions from the next frame of data results in an error that can then be compared to a predetermined threshold, if the error exceeds the threshold the set of data is classified as an anomaly. The rate of falsepositives and false-negatives was then used to determine the effectiveness of the model.

This works hopes to show that structural health monitoring of piping systems can be done with little to no human judgement once a system has been trained. The advantages of such a system would be the ability to process data in real-time and always be continuously monitoring infrastructure for anomalies so that human error can be further reduced. Operator error will always plague work environments and in some industries, it is simply the cost of doing business. In the nuclear industry, however, the costs of poor or negligent 
performance are too high to accept and could result in not only deaths but contamination of the environment for generations to come like what happened in Chernobyl. Other incidents such as Three Mile Island in 1979 were caused because of an abnormal pump behavior which resulted in thousands of gallons of coolant being removed from reactor 2 (TMI-2) before it was noticed [8].

\subsection{Related Work}

Machine learning models are becoming ever more ubiquitous when it comes to automated monitoring applications and promising results have come from applying machine learning models to acoustic sensor technologies for SHM [76] [77] [78]. SHM has been a focused field of study for such a time that fundamental axioms [3] have been put forward to outline the assumptions applied to the science. These assumptions pertain to the capabilities of identifying and quantifying damage to infrastructure and are as follows [3]:

I. All materials have inherent flaws or defects.

II. The assessment of damage requires a comparison between two system states.

III. Identifying the existence and location of damage can be done in an unsupervised learning mode but identifying the type of damage present and the damage severity can generally only be done in a supervised learning mode.

IV.

a. Sensors cannot measure damage. Feature extraction through signal processing and statistical classification is necessary to convert sensor data into damage information. 
b. Without intelligent feature extraction, the more sensitive a measurement is to damage, the more sensitive it is to changing operational and environmental conditions.

V. The length- and time-scales associated with damage initiation and evolution dictate the required properties of the SHM sensing system.

VI. There is a trade-off between the sensitivity to damage of an algorithm and its noise rejection capability.

VII. The size of damage that can be detected from changes in system dynamics is inversely proportional to the frequency range of excitation.

Axioms II - IV strongly agree with the application of machine learning to play a role in damage detection and identification. Further research has already taken this step by the same author as the axioms above, and put forward the argument that machine learning offers a natural framework to address detection, localization, and assess damages [79].

There are several different fundamental types of machine learning models that have so far been applied to SHM. Convolutional neural networks (CNN), a form of deep neural networks originally designed to process image data, have previously been used in image processing, music recommendations [80], image classification, and natural language processing [81]. CNNs are used traditionally on 2-dimensional data like a 2D image, but can also be fed 1-dimensional sequences; this method of 1D CNN has been used in SHM of structures [82] with good results. Other work has focused on the use of recurrent neural networks (RNN) which is a form of artificial neural network that is designed to process temporal sequences of data to solve problems such as speech recognition [83] [84]. While both CNNs and traditional RNNs are capable of handling time-series data, learning long- 
term dependencies has always posed a problem [85] due to the exponential multiplication of weights for deep computational graphs. Long short term memory (LSTM) machine learning models are a form of RNN that is specifically designed for learning long-term dependencies for applications of time series forecasting [86], have been used in many different applications from automotive suspension control and monitoring [87] to novel acoustic signature detection [88] and vocabulary recognition [89]. LSTM models are useful for SHM with acoustic sensors due to the ability to process time series data and learn longterm dependencies. Work recently published by Korean scientists showed that forms of LSTM models could even be used for detecting sensor faults (defects in the data reported by sensors) [90]. Hybrid models combining LSTM and CNN are also becoming popular approaches in SHM [91] do to the ability to combine the benefits of long-term dependency learning in LSTMs and spatial relationship analysis in CNNs.

\subsection{Methods}

\subsubsection{General Architecture}

The implementation of an automated model event detection model has several benefits, one of them being that resources for running the model does not have to be on site, neither human nor machine. With the ability to stream data to a remote machine, pipelines in the Midwest can have data from hundreds of sensors processed on servers on the east coast. The architecture laid out in provides a high-level flow chart of the software components involved in creating an anomaly detection and classification system. 


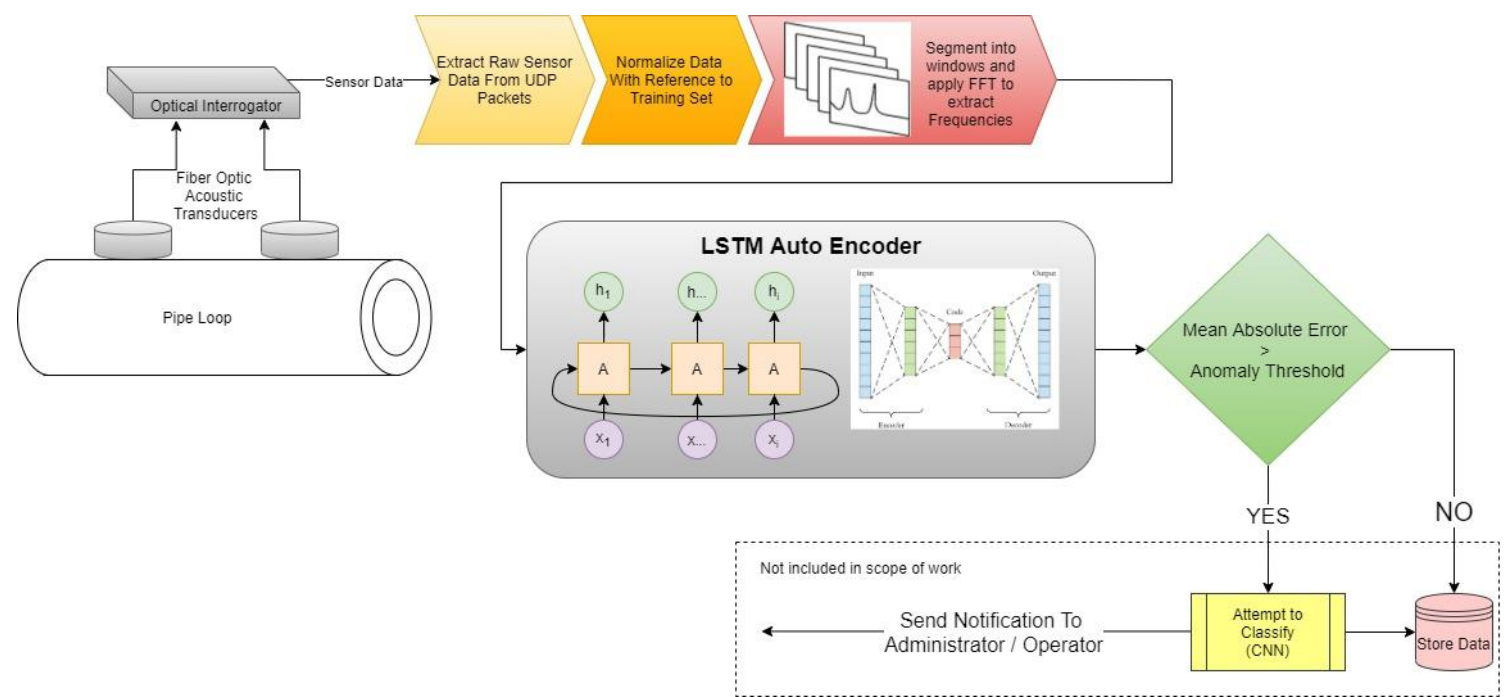

Figure 4.1 - Acoustic Transducer Anomaly Detection MLM Architecture

This work focuses on the first two steps, preprocessing the raw data from the Fiberstrike optical interrogator unit and feeding that into an LSTM machine learning model that will be able to extract features determined to be anomalies. Classification will not be covered in this work but is the logical next step.

\subsubsection{Preprocessing}

Optical transmissions from the fiber optic acoustic sensors are first converted into digital signals by the CEL Fiberstrike LCM-2500 interrogator which then outputs a UDP stream of packets. As described in the previous chapter, each UDP packet contains 16 timeintervals from all sensors connected to the interrogator and each packet has a time interval of approximately $320 \mu$ s giving each sensor a resolution of $50 \mathrm{KHz}$. For the purposes of this research, UDP packets were captured using WireShark and saved to a packet capture file. First a script was written in Python to extract each timestep of sensor data, convert the raw hex bytes to floating point integers, and then save them to file as comma separated values (CSV) for later use. Once the data was extracted, several different techniques were used to 
preprocess the data for the LSTM model to ingest. LSTM models in the Keras library accept 3 dimensional arrays of inputs, (samples, timesteps, features) [92]. Samples are the number of sequences and a batch is one of more sequences. Each timestep is a perspective of observation; a 1D sequence of values may be the result of 5 sequential events repeated continuously, therefore a 1D array of 10 values should be reshaped into a 2D array of 2 samples with 5 timesteps each. Features are the number of observations to be considered by the model each timestep, these are measurements or metrics extracted during preprocessing.

Careful consideration was taken in determining the number of features that the model should base its predictions on as well as the resolution of each sample size. Based on results from extracting features from the raw acoustic data in previous work [65] and mentioned in section 3. Fiber Optic Acoustic Transducers, the two approaches considered were using the raw acoustic signal as the input or applying the fast Fourier Transform and isolating individual frequencies as features.

Works and examples studied implementing LSTM models in Python for anomaly detection [93] [94] relied on the raw vibrational data from accelerometers and built their models around using a single feature for each sensor or channel. The type of data and the type of anomalies being look for plays an important role in the features an MLM is trained on. In the examples mentioned, ball bearings were being monitored for predictions of failure, but since degradation is a slow process the absolute mean was taken of 600 values every 10 minutes, the original resolution being $1 \mathrm{~Hz}$. To test this preprocessing method a controlled data set of ball bearing accelerometer measurements was acquired from the 
NASA Acoustics and Vibration Database [95]. After taking the absolute mean of the data and normalized between 1 and 0 it was graphed in Figure $4.2 \mathrm{a}-\mathrm{b}$ ).

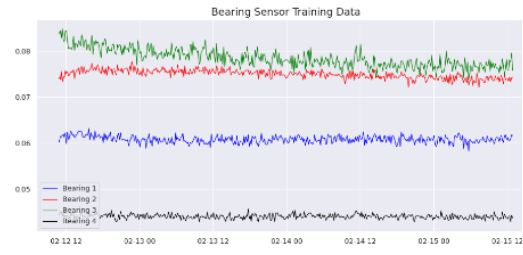

a) Training Data

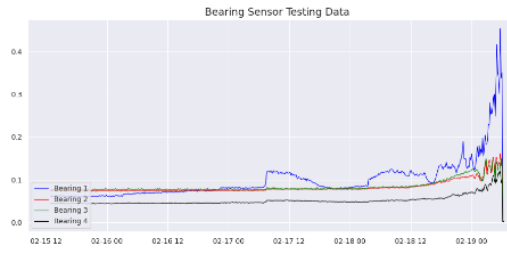

b) Testing Data

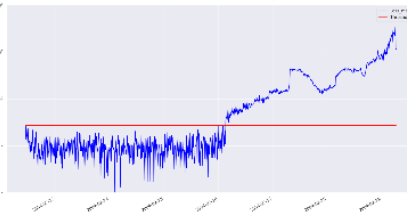

c) Prediction Scoring

Figure 4.2 - MLM model validation on ball bearing acoustics data

From the preprocessed data abnormal behavior is easily identified and the data set ends when the machinery that was being monitored was taken offline because of ball bearing failure, seen as the values all drop to 0 in Figure 4.2 b). An LSTM model was created which will be explained in the next section - and was trained on the data set in Figure 4.2 a) and then fed testing data shown in Figure $4.2 \mathrm{~b}$ ). The resulting mean absolute error (MAE) was graphed in Figure $4.2 \mathrm{c}$ ). The horizontal red line represents the threshold value that serves as the boundary of what is considered an anomaly or not, anything above it is abnormal behavior. This approach was successful, but the original data was far from nuanced and simple linear discrimination applied to the raw testing data would have achieved the same results as the MLM. When this method was applied to acoustic data from the pipeline, the model was less successful at drawing a clear distinction between normal operating behavior and when a leak was occurring. 


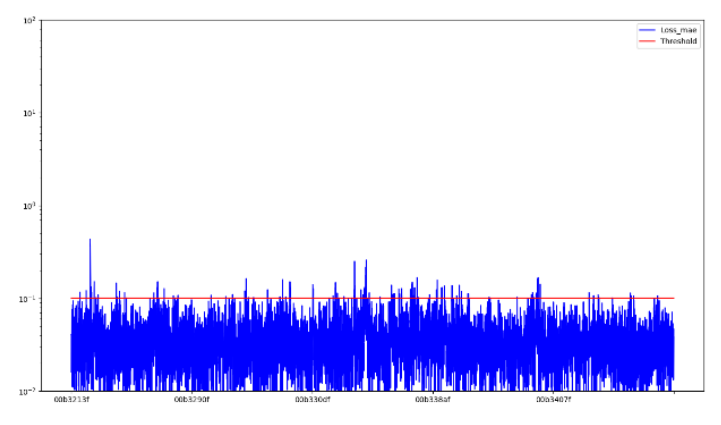

a) Scored Training Data Set

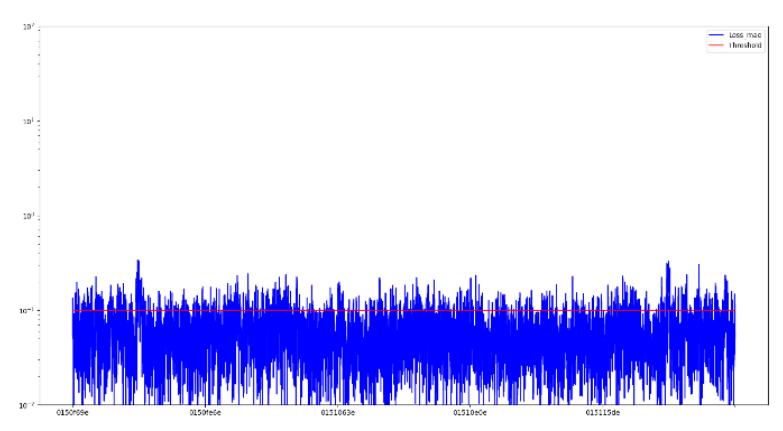

b) Scored Test Data Set

Figure 4.3 - MLM model tested with pipeline acoustics data

The limitations of the model to draw pronounced conclusions may be due to the fact that the bearing data was averaged over much longer periods of time (10 minutes) compared to the acoustic data $(<1 \mathrm{sec})$. Averaging over longer periods of time reduces the effect outliers in the data have on value at each timestep but also results in a slower response time for a model trying to monitor a system in real- time. A 10-minute interval would not be acceptable for leak detection as significant damage may have already occurred and more than 1 interval predicted as an anomaly would be required to verify the event was not a false positive. At 50,000 points per channel per second, processing each timestep from the acoustic sensors would result in the model being too strongly influenced by noise. Taking the absolute mean of different intervals from $1 / 10$ of a second up to 1 second was tested but did not yield promising results.

Based on observations in previous work described in section 3, it was known that different events on the pipeline affected various frequencies differently. A single data point in a raw acoustic signal is the equivalent of the sum of all the energy in the entire frequency spectrum, but by applying the FFT the signal can be split into its frequency subcomponents. 
Therefore, instead of using the raw acoustic signal as from each channel as a single feature in the LSTM, the amplitude of each frequency domain could be used as a single feature.

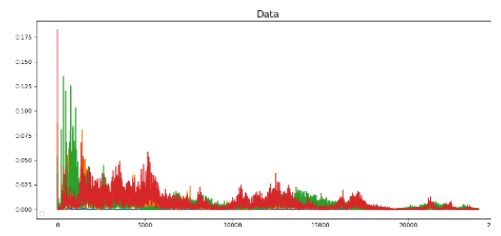

a) Instantaneous FFT of 5000 points from 4 channels

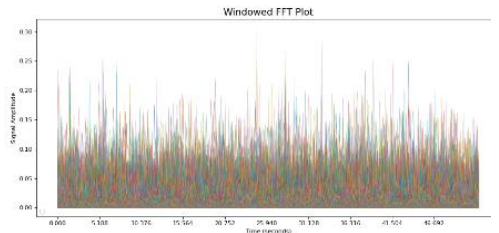

b) Each Frequency plotted against time for 1 channel

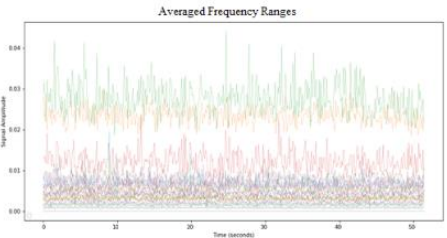

c) Frequencies averaged into 25 ranges

Figure 4.4-FFT applied to acoustic signals for preprocessing

Applying the FFT to a set of $\mathrm{N}$ samples results in amplitudes for $\mathrm{N} / 2$ real and positive frequency segments. Figure 4.4 shows several representations of the frequency extraction from the original acoustic signal; a) represents the instantaneous amplitudes of all extracted frequencies from four sensors, this would represent what would be fed to the LSTM model at each time step, however, just graphing this data from one channel over a period of a minute, $b$ ), shows that just as much noise is present due to 2500 features being plotted. In c), once the mean of 100 contiguous sets of frequencies has been taken, meaningful features can be shown to appear. The FFT cannot be applied to a single time step of raw datapoints as the range of frequencies extracted is represented by the following equation:

$$
f_{i}=i * F_{s} / N
$$

Equation 2 - Frequency at index i of FFT output

Where $\boldsymbol{F}_{\boldsymbol{s}}$ is the sampling frequency in Hertz or 1/sampling rate; $\boldsymbol{N}$ is number of samples. $f_{i}$ represents the frequency in Hertz of the $\boldsymbol{i}_{\text {th }}$ value generated by the FFT. Applying the FFT on $N$ samples will result in $N$ values, however, all values after index $N / 2$ have complex 
conjugate symmetry and do not actually represent amplitudes energies in the corresponding $f_{i}$ calculated frequency. Therefore, $N$ samples yields $(N / 2)-\boldsymbol{1}$ usable results $\left(f_{i}(i=0)=\right.$ $\mathbf{O H z}$ and is not usable) with a resolution of $2 \boldsymbol{F}_{\mathrm{s}} / \boldsymbol{N}$ and the amplitude value at index $i$ represents the sum of all amplitudes between $\boldsymbol{f}_{i-1}$ and $\boldsymbol{f}_{\boldsymbol{i}}$.

With a sampling rate of $50 \mathrm{KHz}$ it is then possible of analyzing the frequency spectrum between $\sim 1-25 \mathrm{KHz}$. Different values were tested for $N$ from 1000-20,000 samples (0.02-0.40 seconds), which would determine the time interval between each sample of features. The resulting $(\boldsymbol{N} / 2)-\boldsymbol{I}$ frequencies were then separated into $\boldsymbol{K}$ features per sensor by averaging each contiguous set of frequencies. These steps can be represented by the following equations:

$$
K_{\max }=N / 2-1
$$

Equation 3 - Maximum number offeatures in array $k$

$$
a=\frac{N / 2-1}{K}
$$

Equation 4 - Number of values averaged per feature in $k$

$$
\left\{f \in \mathbb{R} \mid\left[(i-1)\left(a^{F_{S}} /_{N}\right), i\left(a^{F_{s}}{ }_{N}\right)\right]\right\}
$$

Equation 5 - Frequency range for $i_{\text {th }}$ feature in $k$

$$
k_{i}=\frac{\sum_{n=0}^{a-1}(n+a i)\left(F_{S} / N\right)}{a} \quad i=0,1, \ldots K-1
$$

Equation 6 - Value of each ith feature in $k$ 
It should be noted that $\boldsymbol{K}_{\max }$ is as the maximum number of useable results from the FFT as it would be possible to use every extracted frequency group as a single feature. This was not attempted as it would entail thousands of features and result in exponentially longer training time and complexity for the model when good results could be achieved with less features. A range for $\boldsymbol{K}$ was tested between 2-100 features per channel and after trial and error, the best results were achieved by using a sample size of $N=\mathbf{1 0 , 0 0 0}$ and $K=\mathbf{2 4}$ features per channel. This resulted in each feature representing an increment of approximately $1 \mathrm{KHz}$ between $1-25 \mathrm{KHz}$, each sample of data from all four sensors containing 96 features per sample, and each sample representing 200ms of time.

\subsubsection{MLM Structure and Training}

An autoencoder is a neural network that is designed to reduce a set of data into only the most significant features and then attempt to reconstruct a representation of the original data from the significant features it extracted [96]. By training an autoencoder to accurately recreate data with the same sets of properties these neural networks can be used as good anomaly detectors because data that does not share the same set of core properties will create a reconstruction error that can then be compared against an acceptable threshold for determining if there is an anomaly in the data. The model used consisted of only 5 layers, 2 input layers and two output layers with a layer in between that distributes the compressed features from the input layers across the timesteps of the output side layers. 


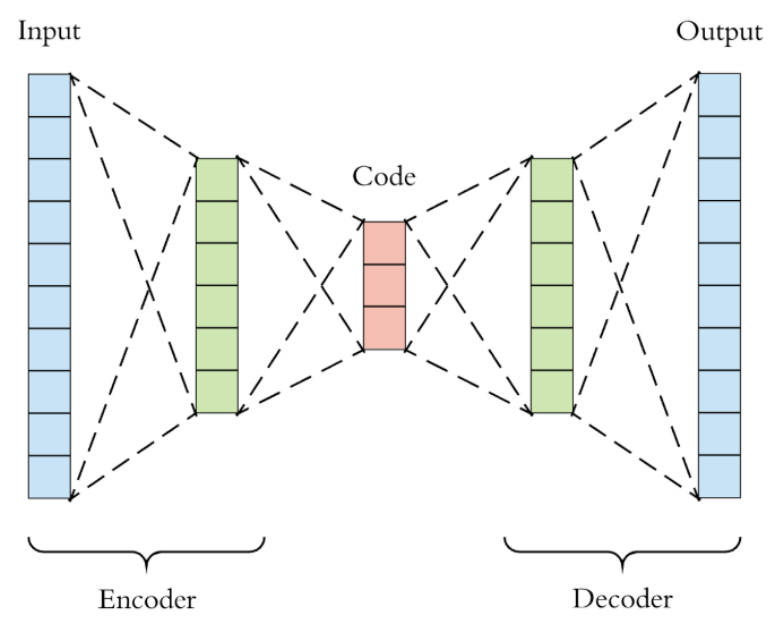

Figure 4.5 - Autoencoder Architecture [97]

This research did not focus strongly on different layer stack ups but a 11-layer and 5-layer autoencoder were tested with the best results coming from the 5 layers model. Each of the input and output layers were creating as LSTM layers and the middle layers as a repeat vector since it merely redistributed the data.

When compiling the model the 'adam' optimizer was chosen due to its flexibility and effectiveness with large datasets [98]. 'adam' is an algorithm for stochastic gradientdescent which is used to achieve the correct shift in weights during the training of the model. While the optimization function is an important part of any machine learning algorithm [99] this research does not compare different optimization functions and uses 'adam' for all results.

The number of neurons per layer is usually dependent on the number of input features [100]. Discussed in section 4.3.2 Preprocessing, the number of input features was experimented with from 4 to 400 , this lead to the need to change the number of neurons on all layers each time. For layers 2 and 4, the last layer of the encoder and the $1^{\text {st }}$ layer of the decoder, the same number of neurons as total input features were used each time and yielded consistent results. The same number of neurons on the input and output layers were 
used so that the shape of the data returned from the autoencoder was directly comparable to the original input. The number of neurons for these outer most layers was primarily tested between 2-4 times the number of input features with 2 times being used to generate the results shown later in this chapter. Higher number of neurons was initially tested but the high computational load and slow training time when the number of neurons was greater than 200 lead to exploration being abandoned in that area. In addition, after 4 times the number of features, increases in anomaly detection was not noticed.

Once the architecture of the neural network was determined, the number of epochs for training the model was determined through graphing the history of recreation error by the autoencoder at each epoch and graphing the results. Figure 4.6 shows the history of the mean absolute error generated by the autoencoder at the end of each training epoch. To calculate this, the output array from the autoencoder is compared directly to the original input array and recreation error is calculated as the absolute average of the subtraction of one array from the other. Keeping a record of these values allows one to see the speed at which the autoencoder can achieve a low level of error on the training data.

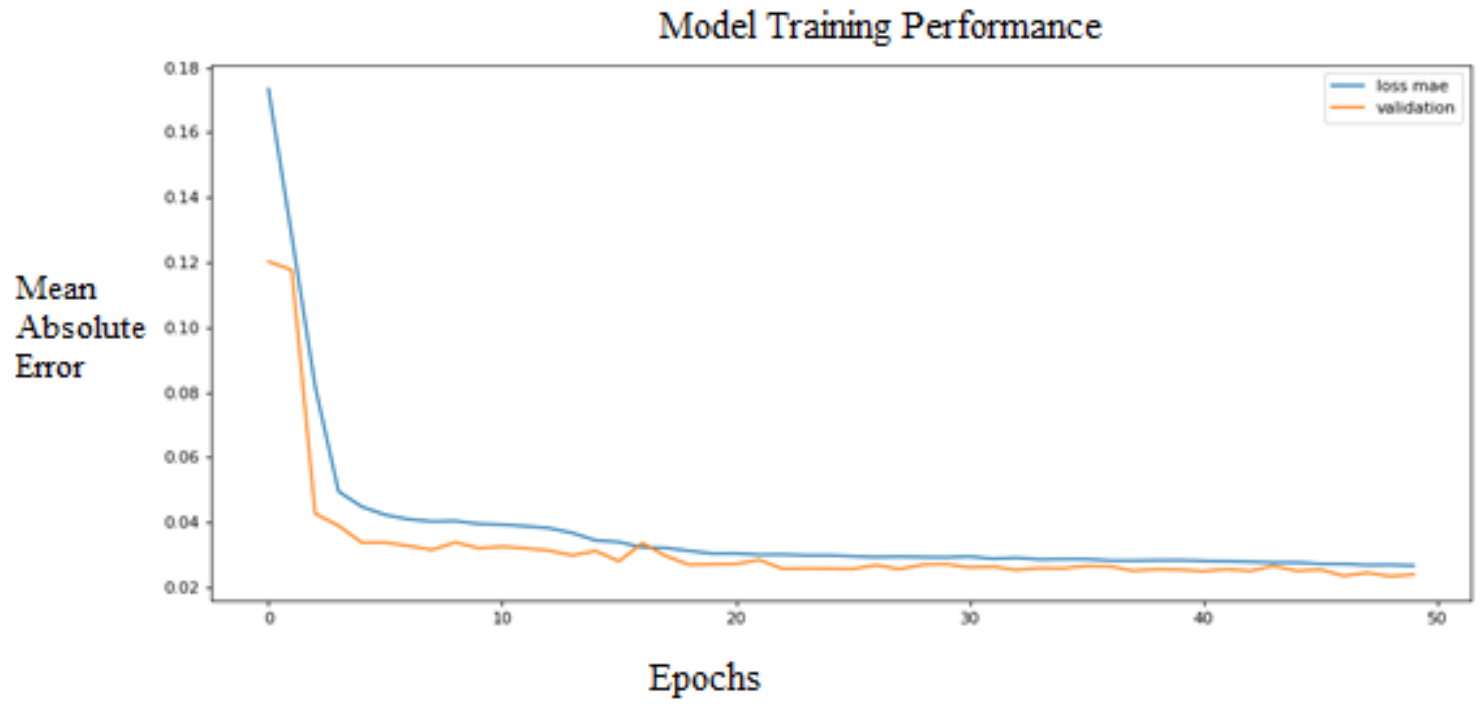


For each epoch, $5 \%$ of the training set was removed during training and then fed into the model to attempt to recreate the data without ever being trained on it. The results can be seen in Figure 4.6 as the series labeled validation. A tight fit between the 'validation' and 'loss_mae' series in Figure 4.6 indicates that the training data is homogenous and that the model is not merely memorizing the datasets it is being shown. The model was trained for 50 epochs for all the following results due to its repeated ability to achieve low recreation errors across multiple weight randomizations. When trained for only 20-30 epochs, the model produced higher than expected mean absolute errors when asked to evaluate the original training data again and when the model was trained for higher amount of epochs (>100), it began to memorize the original dataset which resulted in false negatives when shown data it wasn't trained on but had no anomalies.

\subsubsection{Anomaly Predictions}

Inherently, and autoencoder does not 'detect' anything, instead it recognizes things based on a smaller-than-input number of features. The autoencoders excellent ability to recognize patterns in data it has seen before can then be used to discern an anomaly when the neural network fails to recognize something either partially or completely. The model itself does not actually flag an anomaly but instead outputs a prediction of what the input data looks like based on the internal features it extracted. Using classical programming methods, the output array is compared to the input array to determine the mean absolute error. An autoencoder is never expected to be perfect and will always generate an error, however, on patterns it is trained on this error should be small. By quantifying the 
maximum error that non-anomalous data generates, a threshold value can be determined that will serve as a boundary between an anomaly and normal behavior.

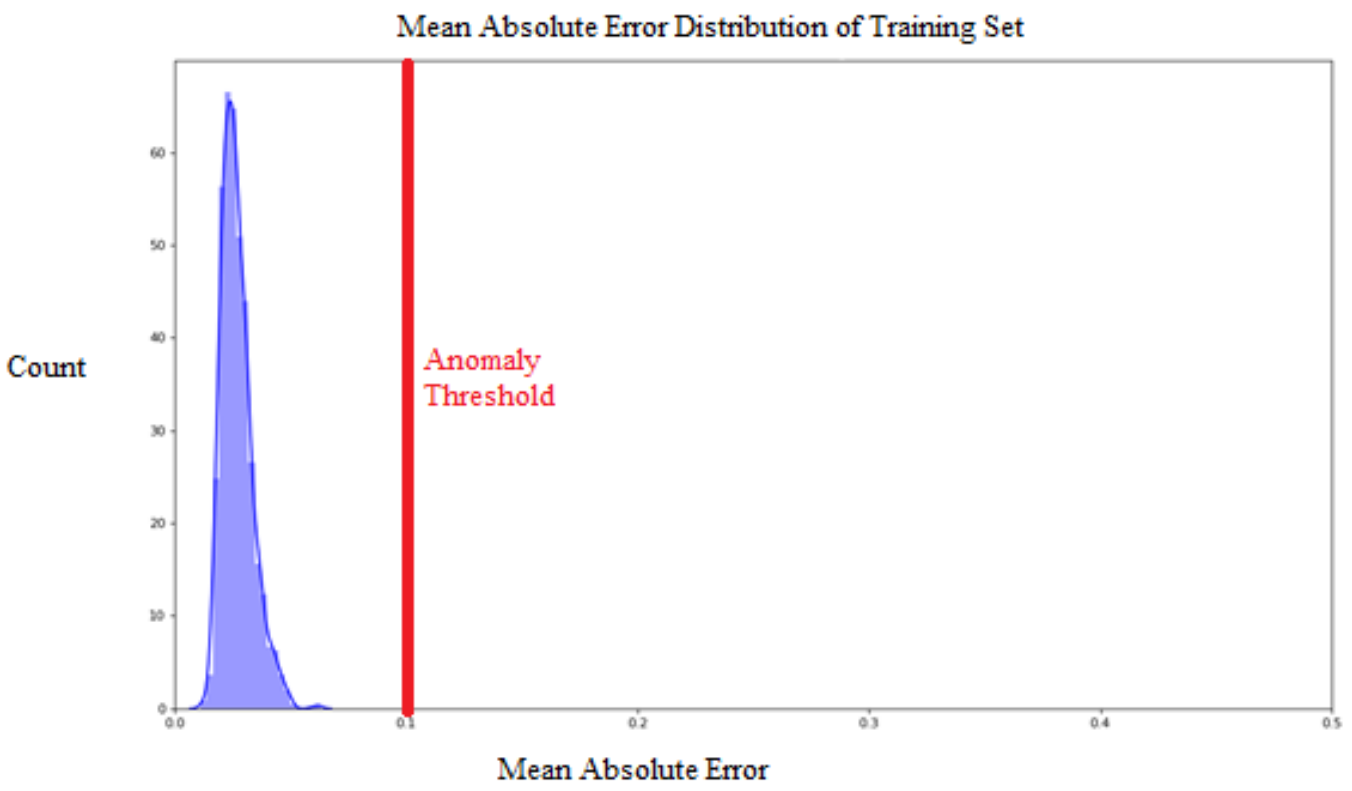

Figure 4.7 - Distribution of autoencoder recreation error of training data

After training the model for 50 epochs on non-anomalous data, the same data was then fed to the model for prediction and the mean absolute error of the results were taken for each sample. Figure 4.7 shows a distribution plot for the error values generated from this test to determine a viable threshold value, which if crossed, will indicate an anomaly. From the graph it can be seen that no values are greater than approximately 0.75 , but to add a small buffer for false positives, a threshold value of 0.1 was decided.

To isolate and graphically indicate where the model had identified an anomaly a simple algorithm was coded to find the indexes of error values that crossed the threshold $y$-value. Dashed vertical red lines were then graphed to indicate the presence of an anomaly in that area. 


\subsection{Results and Discussion}

To keep the results of the MLM anomaly detection comparable to previous work shown in section 3 - Fiber Optica Acoustic Sensors, where acoustic data was analyzed for visual patterns, the same data sets described there were used to train and test the anomaly detection model. The graphs shown below are a concatenation of the original training data, the end of which is indicated by a black vertical line, and the data set that is being tested for anomaly detection. Dashed red vertical lines indicate an anomaly, meaning that when analyzing the data, the resulting error was greater than the preset threshold of 0.1 .

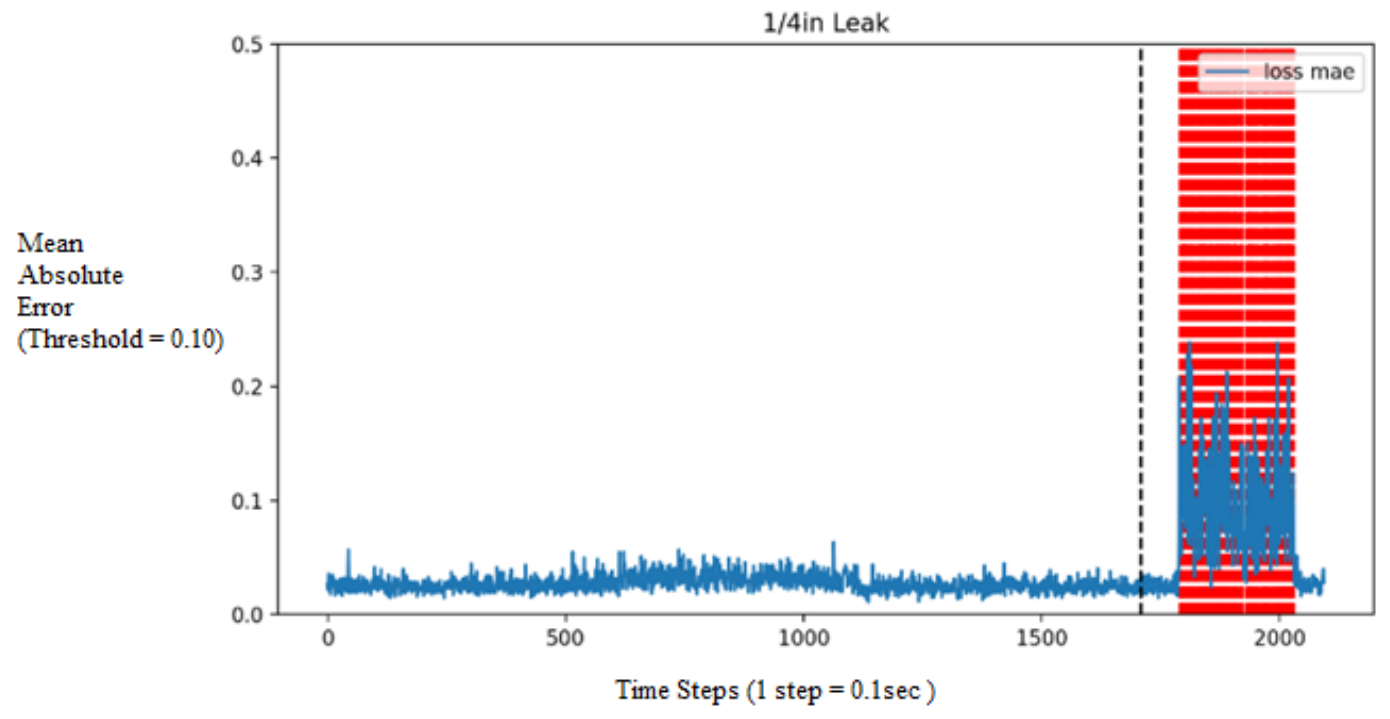

Figure 4.8 - MLM Identifying 1/4” Leak

One of the main goals of developing this model was for automated leak detection. In Figure 4.8 we see the results of the model when shown the same data from section Figure 3.17 which was characterized as a $1 / 4 "$ holed opened in a vertical section of pipe in between sensors 1 and 2, the dataset was a recording of normal operation with the leak occurring after the recording started and being stopped before it ended. Figure 4.8 clearly shows where the model flagged the anomalous data caused while the leak was active but did not 
detect anomalies on either side as the pipeline remained in its healthy operating condition. Of all the sets of data, however, the $1 / 4$ " leak showed one of the most noticeable signatures and it was expected for the model to perform well on it.

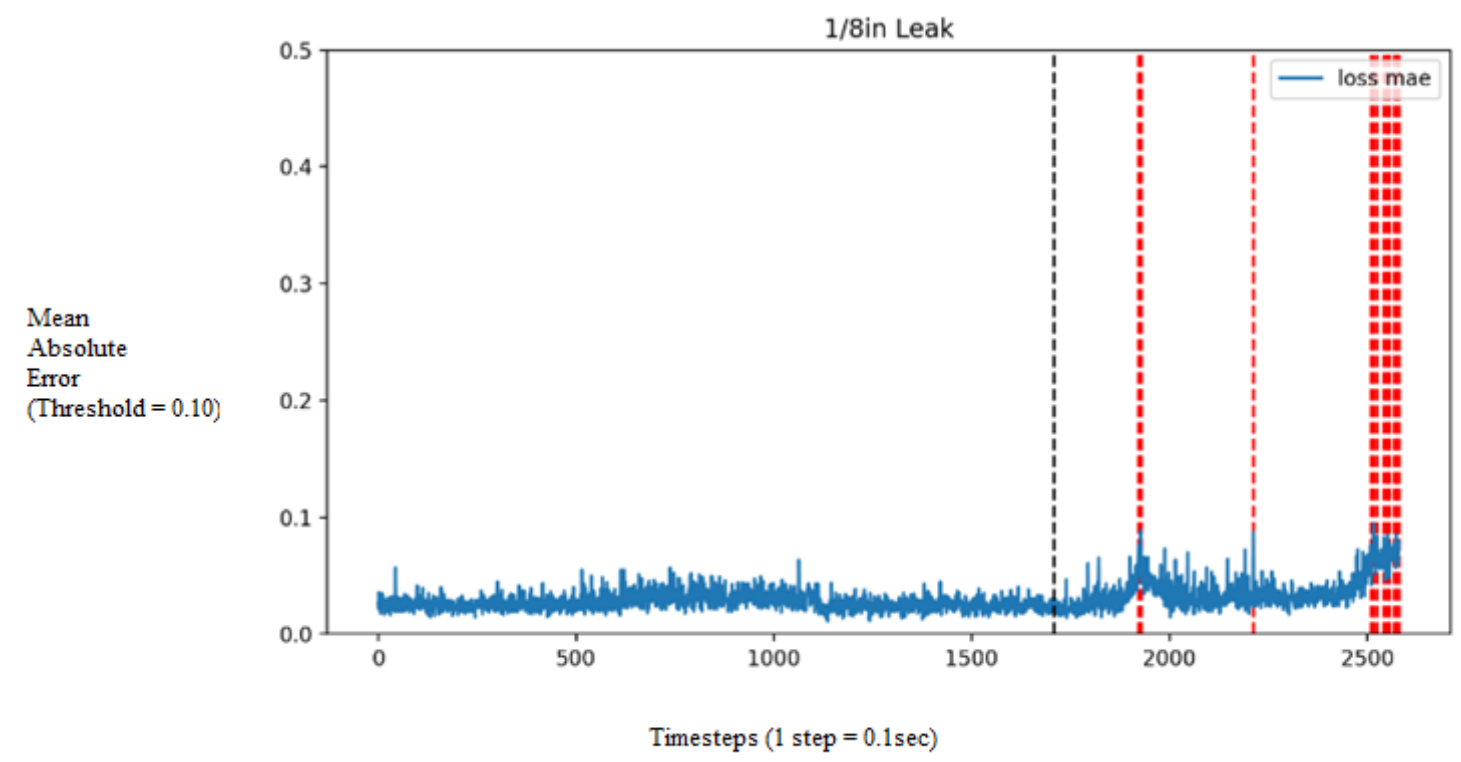

Figure 4.9 - MLM identifying 1/8" Leak

Another set of data that was of considerable interest was that of the smaller $1 / 8$ " leak in the vertical section that was characterized as a normal operation before the hole was opened and then left open for the duration of the data collection set. Figure 4.9 shows the anomaly detection results of analyzing the dataset first described in Figure 3.21. The model accurately labels the initial opening of the leak as an anomaly but does not flag several sections of the data when the leak is occuring as an anomaly. Discussed in the previous chapter, the leak exhibitted different behaviour than the $1 / 4$ " leak by pulling a vaccum and pulling in air as opposed to expelling water constantly. Despite the fact that the graphs of raw data and even the windowed FFT plot showed very little significant deviations from 
normal operation, the model still flagged a significant portion of the data as anomalous which shows promising results.

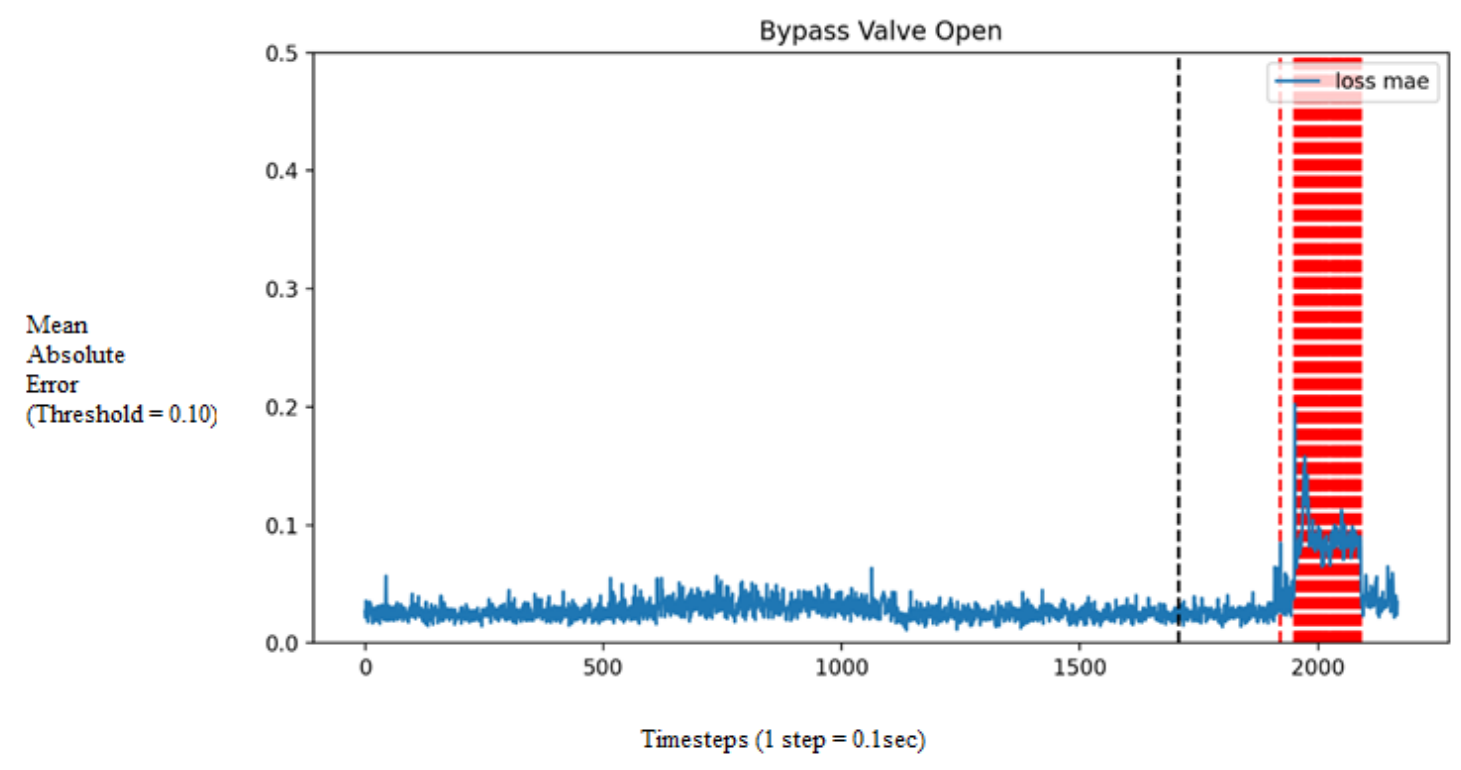

Figure 4.10 - MLM identifying bypass valve opening

As mentioned in previous sections, mechanical failure is not the only events on a pipeline that can lead to catastrophic results. Routine actions such as opening and closing valves, when done at the wrong time, can lead to disasters. That was why it was important to test the ability of the model to not only identify faults such as leaks, but fundamental changes in operation. Figure 4.10 shows the model was able to clearly detect the change in flow when the bypass valve was opened and then closed during normal operation. The dataset tested on is the same as Figure 3.23 which showed clear deviation from normal behavior while the bypass valve was open but then a return to normal behavior once it was closed. 


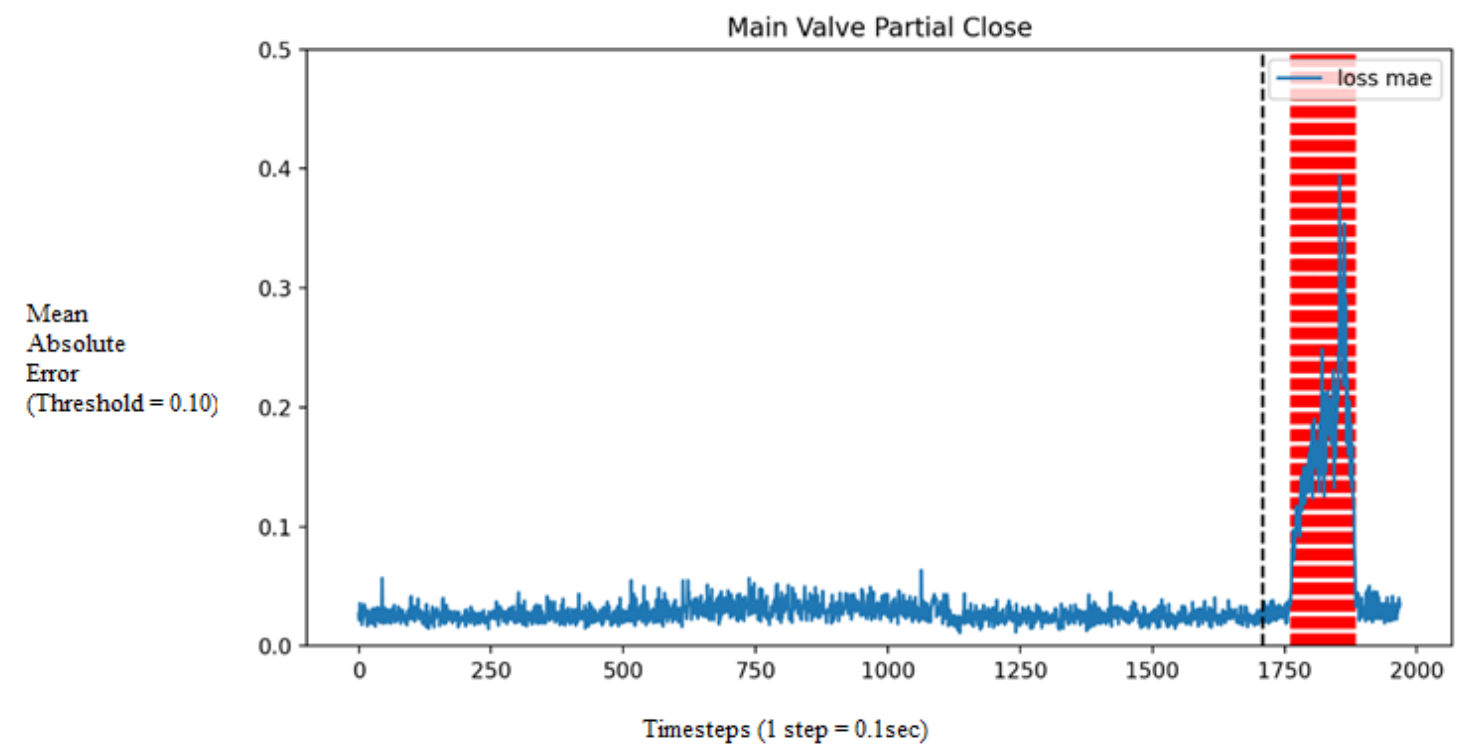

Figure 4.11 - MLM identifying main valve partially closed

In another test the main valve that leads to the overhead stretch of pipe on the test loop was closed partially to simulate a blockage or incomplete valve operation. The dataset shown in Figure 3.25 was analyzed by the anomaly detection model and perfectly flagged the anomalous behavior caused by the valve being partially closed, seen in Figure 4.11.

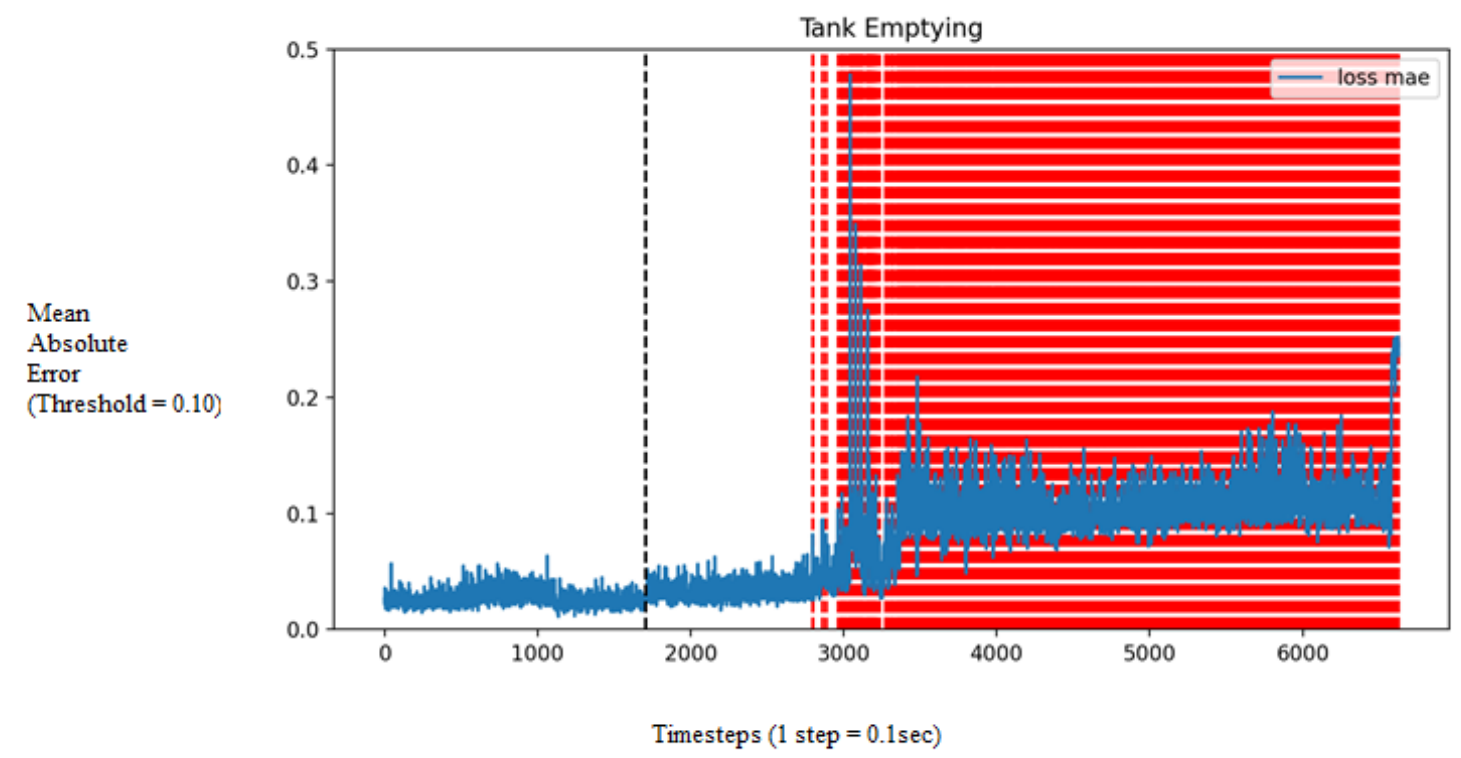

Figure 4.12 - MLM Identifying abnormal behavior while tank empties 
Another test that showed extremely promising results was one done to see if changes in water level in the tank would be identifiable by the model even though the pipeline was operating under normal conditions. While recording normal operation, a hose was used to slowly siphon water from the 80-gallon plastic tank acting as the reservoir and return for the pipe loop. The data collection occurred until there was no longer enough water in the tank for the input pipe to the pump to be fully submersed at which point the test had to be stopped to protect the pump from damage. Figure 4.12 shows the MLMs analysis of the data collected while the tank emptied. The tank began full and emptied at a steady rate which indicates that once the tank was less than about $80 \%$ full, the change in pressure in the system caused by the lower water level was detectable by the model. Several factors could have caused the change in acoustic signal that caused the anomaly such as a higher strain on the pump due to lower water pressure at the input. Another factor may have been the water level dropping below the level of the output pipe, causing water to splash into the tank and causing more noise. Another significant aspect in Figure 4.12 is the sudden spike in the last 200 hundred samples of the data, this was caused by the input to the pump becoming unsubmerged and ingesting air. The increase in error score indicates that the model might also discriminate other pump operation irregularities exceedingly well and this aspect should be investigated further.

\subsection{Conclusion}

The results of this work have shown favorably that an automated anomaly detection model based on an LSTM-autoencoder neural network can effectively discriminate irregular pipeline behavior. Of the five test sets of data shown in the results section and two others not shown, the model did not produce any false positive results. The neural 
network did return some false negatives by not flagging an entire region of anomalous data as seen in Figure 4.9, however, this lapse in detection could easily be overcome through classical programming means as significant portions of the anomalous data was flagged but not all of it. Similar behavior occurred when running the model on data sets from open leaks, but in every case the model still flagged significant portions of the anomalous data. In these tests leaks were manually controlled and left active for no more than several minutes at a time, in a real world scenario a leak will be continuous until it is fixed or the piping system taken offline; the model would have significant more opportunities to flag the anomalous behavior and false negatives witnessed may only manifest as a slight delay before an alert notification is triggered.

During initial training and testing of the model a consistent problem was the appearance of false negatives in datasets that were not trained on but exhibited no anomalies. The solution was to increase the number and diversity of the samples of data used for training. If the data set used for training was from a single day it was more common for false positives to appear in data collected on different days. By combining segments of data from as many periods of time as possible to create a single training the appearance of false positives was eliminated completely in all test sets of data used. It is also important to point out that when compiling the training set of data that sample sizes of new segments from different periods needed to be of a statistically significant size to raise the mean absolute error during each epoch otherwise the model would ignore its failures to accurate predict the outcome of the dataset. It was found that individual datasets could be significant larger than the majority of training sets spliced together but sample sizes smaller than the 
median resulted in poor performance and an the model flagging the same data as anomalous even after training for many epochs.

Environmental factors that change daily or seasonally that affect the propagation of sound such as water temperature, atmospheric pressure, background noise, and humidity may result in higher chances of false positives when the model has not been trained on data generated during those environmental conditions. This would explain the inability of the model to immediately recognize healthy data if it was not collected near the same time as the original training set. If this hypothesis were true it would suggest that the implementation of a machine learning model similar to the one in this research may require consistent maintenance to constantly update its training with data from different weather and atmospheric conditions.

This research has shown that the CEL Fiberstrike fiber optic acoustic transducers make for a potential candidate when implementing an automated pipeline monitoring system. A major advantage of the acoustic transducers being their universal ability to detect any event that registers a vibration. Pipeline faults, routine actions, and nearby excitations can be monitored via a single sensor and interpreted through the same software model which significantly reduces the logistics of monitoring system installation and upkeep. Further research is still required to test the full capabilities and the Fiberstrike sensors; they must also be compared to other sensors on the market both in performance and cost before any recommendations can be made for a full monitoring system to be developed around them.

The next steps in validating this research will be to expand both the training and testing data sets and subject the machine learning model to rigorous performance tests to quantify its reliability. If needed, classical programming techniques may need to be implemented to 
augment the machine learning model to bridge shortcomings in the pure machine learning nature of the software. In addition, the program should be extended to incorporate classification MLMs that can determine the identity of anomalous events that are flagged by the LSTM-autoencoder. Overall, the model has achieved all its intended goals and correctly identified leaks both small and large in very noisy environments as well detected as subtle changes such as changes in water pressure from changes in tank water level. 


\section{REFERENCES}

[1] ASME, "API 579-1/ASME FFS-1 Fitness for Service," AMSE, 2007.

[2] D. McDaniel, J. Arniella, B. Castillo, D. Washenfelder and J. Engeman, "Analysis of Erosion/Corrosion Data for High-Level Waste Pipelines at Hanford," in Proceedings of the Waste Management Symposia 2015, Phoenix, AZ, 2015.

[3] K. Worden, C. R. Farrar, G. Manson and G. Park, "The Fundamental Axioms of Structural Health Monitoring," Philosophical Transactions of the Royal Society A, vol. 463, pp. 1639-1664, 3 April 2007.

[4] Nuclear Engery Institute, "Nuclear Costs in Context," Nuclear Energy Institute, 2018.

[5] S. Dipen, "Acoustic Sensor for Pipeline Monitoring," Los Alamos National Laboratory, Los Alamos, 2005.

[6] E. Cardis, M. Vrijheid, M. Blettner and et al, "The 15-Country Collaborative Study of Cancer Risk Among Radiation Workers in the Nuclear Industry: Estimates of Radiation-Related Cancer Risks," Radiation Res., vol. 167, no. 4, pp. 396-416, 2007.

[7] M. Schubauer-Berigan, R. Daniels, S. Bertke, C. Tseng and D. Richardson, "Cancer Mortality Through 2005 Among a Pooled Cohort of U.S. Nuclear Workers Exposed to External Ionizing Radiation," Radiation Res, vol. 183, no. 6, pp. 620-631, 2015.

[8] Office of Inspection and Enforcement, "Investigation into the March 28, 1979 Three Mile Island Accident," U.S. Nuclear Regulatory Commission, Washington D.C., 1979.

[9] A. Barrias, J. R. Casas and S. Villalba, "A Review of Distributed Optical Fiber Sensors for Civil Engineering Applications," Sensors (Basel), p. 748, 2016.

[10] L. C. Lynnworth, Ultrasonic Measurements for Process Control: Theory, Techniques, Applications, Academic Press, 2013. 
[11] M. Lim and H. Cao, "Combining multiple NDT methods to improve testing effectiveness," Construction and Building Materials, vol. 38, pp. 1310-1315, 2013.

[12] Savannah River National Laboratory, "SRNL Tech Briefs Ultrasonic Thickness," [Online]. Available: https://srnl.doe.gov/tech_transfer/tech_briefs/SRNL_TechBriefs_UltrasonicThic knessMassLossMeasurement.pdf. [Accessed 1 May 2020].

[13] F. Honarvar, F. Salehi, V. Safavi, A. Mokhtari and A. N. Sinclair, "Ultrasonic monitoring of erosion/corrosion thinning rates in industrial piping systems," Ultrasonics, vol. 53, no. 7, pp. 1251-1258, 2013.

[14] R. Jones and T. Jones, "Investigations of sand-water induced erosive wear of AISI 304L stainless steel pipes by pilot-scale and laboratory-scale testing," Wear, vol. 255, no. 1-6, pp. 206-218, 2003.

[15] H. Mansoori, R. Mirzaee and M. A., "Pitting Corrosion Failures of Natural Gas Transmission Pipelines," in Conference Proceedings, IPTC 2013: International Petroleum Technology Conference, 2013.

[16] R. Wood, Y. Puget, K. Trethewey and K. Stokes, "The performance of marine coatings and pipe materials under fluid-borne sand erosion," Wear, vol. 219, no. 1, pp. 46-59, 1998.

[17] K. Edalati, N. Rastkhah, A. Kermani, M. Seiedi and A. Movafeghi, "The use of radiography for thickness measurement and corrosion monitoring in pipes," International Journal of Pressure Vessels and Piping, vol. 83, no. 10, pp. 736741, 2006.

[18] F. Niese, A. Yashan and H. Willems, "Wall Thickness Measurement Sensor for pipeline inspection using EMAT technology in combination with pulsed eddy current and mfl," in European Conference on Non-Destructive Testing, 2006.

[19] M. Parsi, K. Najmi, F. Najafifard, S. Hassani, B. S. McLaury and S. A. Shirazi, "A comprehensive review of solid particle erosion modeling for oil and gas wells and pipelines applications," Journal of Natural Gas Science and Engineering, vol. 21, pp. 850-873, 2014. 
[20] Erosion/Corrosion Research Center, "Erosion/Corrosion Research Center at University of Tulsa," University of Tulsa, [Online]. Available:

http://www.ecrc.utulsa.edu/. [Accessed 1 June 2020].

[21] H. Meng and K. Ludema, "Wear models and predictive equations: their form and content," Wear, Vols. 181-183, pp. 443-457, 1995.

[22] E. Gharaibah, J. Friedemann and N. Davidsson, "Flow Assurance Aspects of Intrusive Erosion Probes," in Offshore Technology Conference, Rio de Janeiro, 2011.

[23] P. Zhang, S. Zheng, J. Jing, Y. Zhou, Q. Li, K. Wang, N. Lv and N. Sun, "Surface erosion behavior of an intrusive probe in pipe flow," Journal of Natural Gas and Engineering, vol. 26, pp. 480-493, 2015.

[24] J. Krautkrämer and H. Krautkrämer, "The Pulse-Echo Method; Design and Performance of a Pulse-Echo Flaw Detector," in Ultrasonic Testing of Materials, Berlin, Springer,, 1990, pp. 167-221.

[25] Olympus Corporation, "V260-SM," Olympus Corporation, [Online]. Available: https://www.olympus-ims.com/en/shop/item/269-productId.570437480.html. [Accessed March 2020].

[26] Z. Parsons and W. Staszewski, "Nonlinear acoustics with low-profile piezoceramic excitation for crack detection in metallic structures," Smart Materials and Structures, vol. 15, no. 4, 2006.

[27] Micronor, "Multi-Axis Fiber Optic Accelerometer," Photon Control, [Online]. Available: https://micronor.com/product/mr660-accelerometer/. [Accessed June 2020].

[28] OptaSense, "Pipeline Monitoring," Qinetiq, [Online]. Available: https://www.optasense.com/pipeline-monitoring/. [Accessed June 2020].

[29] Micronor, "TS Temperature Sensors," Micronor, [Online]. Available: https://micronor.com/products/ts-temperature-sensors/. [Accessed June 2020]. 
[30] FBGS Technologies, "FBG Principle," FBGS Technologies, [Online]. Available: https://fbgs.com/technology/fbg-principle/. [Accessed June 2020].

[31] J. Teixeira, I. Leite, S. Silva and O. Frazao, "Advanced fiber-optic acoustic sensors," Photonic Sensors, vol. 4, pp. 198-208, 2014.

[32] O. Deparis, D. Griscom, P. Mégret, M. Decréton and M. Blondel, "Influence of the cladding thickness on the evolution of the $\mathrm{NBOHC}$ band in optical fibers exposed to gamma radiations," Journal of Non-Crystalline Solids, vol. 216, pp. 124-128, 1997.

[33] M. Kyoto, Y. Chigusa, M. Ohe, H. Go, M. Watanabe, T. Matsubara, T. Yamamoto and S. Okamoto, "Gamma-ray radiation hardened properties of pure silica core single-mode fiber and its data link system in radioactive environments," Journal of Lightwave Technology, vol. 10, no. 3, pp. 289-294, 1992.

[34] j-fiber, "Radiation-hard Fibers," j-fiber, [Online]. Available: https://www.jfiber.com/en/products-services/special-optical-fibers/radiation-hard-fibers/. [Accessed June 2020].

[35] Leoni, "Singlemode and multimode fibers for use with high levels of radiation," Leoni, [Online]. Available: https://www.leoni-fiber-optics.com/en/products-andservices/fibers/radiation-hard-fibers/. [Accessed June 2020].

[36] D. Aggelis, N. Barkoula and A. Paipetis, "Acoustic structural health monitoring of composite materials : Damage identification and evaluation in cross ply laminates using acoustic emission and ultrasonics," Composite Science and Technology, vol. 72, no. 10, pp. 1127-1133, 2012.

[37] A. Behnia, H. Chai and T. Shiotani, "Advanced structural health monitoring of concrete structures with the aid of acoustic emission," Construction and Building Materials, vol. 65, pp. 282-302, 29 August 2014.

[38] R. Finlayson, M. Friesel, M. Carlos, P. Cole and J. Lenain, "Health monitoring of aerospace structures with acoustic emission and acousto-ultrasonics," in 15th World Conference on NDT, Rome, 2000. 
[39] Q. Han, J. Xu, A. Carpinteri and G. Lacidogna, "Localization of acoustic emission sources in structural health monitoring of masonry bridge," Struct. Control Health Monit., vol. 22, pp. 314-329, 2014.

[40] B. Al-Habaibeh and S. A., "Detection of water leakage in buried pipes using infrared technology; A comparative study of using high and low resolution infrared cameras for evaluating distant remote detection," in IEEE Jordan Conference on Applied Electrical Engineering and Computing Technologies (AEECT), Amman, 2015.

[41] M. Lowe, D. Alleyne and P. Cawley, "Defect detection in pipes using guided waves," Ultrasonics, vol. 36, no. 1-5, pp. 147-154, 1998.

[42] M. V. C. Ponce, L. E. G. Castañón and V. P. Cayuela, "Model-based leak detection and location in water distribution networks considering an extendedhorizon analysis of pressure sensitivities," Hydroinformatics, vol. 16, no. 3, pp. 649-670, 1 May 2014.

[43] H. Fuchs and R. Riehle, "Ten years of experience with leak detection by acoustic signal analysis," Applied Acoustics, vol. 33, no. 1, pp. 1-19, 1991.

[44] Y. Gao, M. Brennan and P. Joseph, "A comparison of time delay estimators for the detection of leak noise signals in plastic water distribution pipes," Journal of Sound and Vibration, vol. 292, no. 3-5, pp. 552-570, 9 May 2006.

[45] L. Young and Y. Dong, "An algorithm for leak point detection of underground pipelines," in Proceedings of the Korean Society for Nondestructive Testing Spring Meeting 2004, Seoul, 2004.

[46] D.-B. Yoon, J.-H. Park and S.-H. Shin, "Improvement of cross-correlation technique for leak detection of a buried pipe in a tonal noisy environment," Nuclear Engineering and Technology, vol. 44, no. 8, 2012.

[47] D. Krohn, "Photonics," September 2015. [Online]. Available: https://www.photonics.com/images/Web/Webinars/80/Fiber_Optic_Sensors_Fun damentals_and_Applications.PDF. [Accessed June 2020]. 
[48] J. F. Ready, "Fiber Optics," in Industrial Applications of Lasers, Academic Press, 1997, pp. 530-545.

[49] J. P. Kurmer, S. A. Kingsley, J. S. Laudo and S. J. Krak, "Distributed fiber optic acoustic sensor for leak detection," in OE Fiber, Boston, 1991.

[50] V. Spirin, M. Shlyagin, S. Miridonov, F. M. Jiménez and R. L. Gutiérrez, "Fiber Bragg grating sensor for petroleum hydrocarbon leak detection," Optics and Lasers in Engineering, vol. 32, no. 5, pp. 497-503, 1999.

[51] S.-C. Huang, W.-W. Lin, M.-T. Tsai and M.-H. Chen, "Fiber optic in-line distributed sensor for detection and localization of the pipeline leaks," Sensors and Actuators A: Physical, vol. 135, no. 2, pp. 570-579, 2007.

[52] M. Nikles, "Long-distance fiber optic sensing solutions for pipeline leakage, intrusion, and ground movement detection," in Proc. SPIE 7316, Fiber Optic Sensors and Applications VI, Orlando, 2009.

[53] J. P. Kurmer, S. A. Kingsley, J. S. Laudo and S. J. Krak, "Applicability of a novel distributed fiber optic acoustic sensor for leak detection," in Proceedings Volume 1797, Distributed and Multiplexed Fiber Optic Sensors II, Boston, 1992.

[54] S. Wang, P. Lu, L. Liu, H. Liao, Y. Sun, W. Ni, X. Fu, X. Jiang, D. Liu, J. Zhang, H. Xu, Q. Yao and Y. Chen, "An Infrasound Sensor Based on Extrinsic Fiber-Optic Fabry-Perot Interferometer Structure," IEEE Photonics Technology Letters, vol. 28, no. 11, pp. 1264-1267, June 2016.

[55] The Cleveland Electric Laboratories Co, "FiberStrike optical sensing technology for pipeline monitoring," August 2016. [Online]. Available: http://www.clevelandelectriclabs.com/wp-content/uploads/2016/08/ClevelandElectric-Labs-Marketing-Pipeline-Monitoring-with-Fiber-Optics.pdf.

[56] "FiberStrike Perimeter Security Platform," The Cleveland Electric Laboratories, [Online]. Available: http://clevelandelectriclabs.com/fiberstrike/. [Accessed June 2020]. 
[57] GeeksforGeeks, "User Datagram Protocol (UDP)," GeeksforGeeks.com, [Online]. Available: https://www.geeksforgeeks.org/user-datagram-protocoludp/\#: :text=User\%20Datagram\%20Protocol\%20(UDP)\%20is,connection\%20pr ior\%20to\%20data\%20transfer.. [Accessed May 2020].

[58] Wireshark, "WireShark," Wireshark, [Online]. Available: https://www.wireshark.org/. [Accessed 3 June 2020].

[59] NumPy, "NumPy," [Online]. Available: https://numpy.org/. [Accessed 3 June 2020].

[60] Pandas, "pandas - Python Data Analysis Library," NumFOCUS, 28 May 2020. [Online]. Available: https://pandas.pydata.org/. [Accessed 3 June 2020].

[61] J. Hunter, E. Dale and M. Droettboom, "Matplotlib: Python Plotting," Sphinx, 8 April 2020. [Online]. Available: https://matplotlib.org/. [Accessed 3 June 2020].

[62] K. Newt, "PyShark," GitHub, [Online]. Available:

https://kiminewt.github.io/pyshark/. [Accessed 3 June 2020].

[63] Wireshark, "tshark - The Wireless Network Analyzer 3.2.4," Wireshark, [Online]. Available: https://www.wireshark.org/docs/man-pages/tshark.html. [Accessed 3 June 2020].

[64] T. Finley, "Two's Compliment," 2000. [Online]. Available: https://www.cs.cornell.edu/ tomf/notes/cps104/twoscomp.html. [Accessed 3 June 2020].

[65] A. Aravelli, M. Thompson and e. a. , "Advanced Fiber Optic and Ultrasonic Sensor Systems for Structural Health Monitoring of Pipes in Nuclear Waste Sites," in International Microelectronic Assembly and Packaging Symposia, Boston, MA, 2019.

[66] M. T. Heideman, D. H. Johnson and S. C. Burrus, "Gauss and the History of the," IEEE ASSP MAGAZINE, pp. 14-21, October 1984.

[67] M. Lohne, "The Computational Complexity of the Fast Fourier Transform," University of Oslo, Oslo, 2017. 
[68] O. C. W. T. Hunaidi, "Acoustical Characteristics of leaks in plastic water pipes," Applied Acoustics, vol. 58, no. https://doi.org/10.1016/S0003-682X(99)00013-4, pp. 235-254, 1999.

[69] NumPy, "numpy.fft.fft - NumPy V1.18 manual," The SciPy community, 24 May 2020. [Online]. Available: https://numpy.org/doc/stable/reference/generated/numpy.fft.fft.html. [Accessed June 2020].

[70] Statistics Solutions, "Correlation (Pearson, Kendall, Spearman)," Statistics Solutions, 2020. [Online]. Available: https://www.statisticssolutions.com/correlation-pearson-kendall-spearman/. [Accessed May 2020].

[71] EECS WSU, "Hydrophones [Palouse RoboSub Technical Documentation]," Washington State University, [Online]. Available: http://robosub.eecs.wsu.edu/wiki/ee/hydrophones/start. [Accessed June 2020].

[72] J. H. Cheong, "Four Ways to quantify synchrony between time series data," Medium, 3 May 2019. [Online]. [Accessed 6 June 2020].

[73] Z. Liu and Y. Kleiner, "State of the art review of inspection technologies for condition assessment of water pipes," Measurement, vol. 46, no. 1, pp. 1-15, January 2013.

[74] General Electric, "Sound Speed and Pipe Size Data," 2014. [Online]. Available: https://www.rshydro.co.uk/files/SNSP-and-Pipe-Data.pdf. [Accessed May 2020].

[75] M. Mohri, A. Rostamizadeh and A. Talwalkar, Foundations of Machine Learning, Cambridge: The MIT Press, 2018.

[76] Z. Jiang, Z. Zhang and A. Maxwell, "Extraction of structural modal information using acoustic sensor measurements and machine learning," Journal of Sound and Vibration, vol. 450, pp. 156-174, 2019. 
[77] Y. Ying, J. Garret, I. Oppenheim and L. Soibelman, "Toward Data-Driven Structural Health Monitoring: Application of Machine Learning and Signal Processing to Damage Detection," Journal of Computing in Civil Engineering, vol. 27 , no. $6,2013$.

[78] K. Smarsly, K. Dragos and J. Wiggenbrock, "Machine learning techniques for structural health monitoring," in 8th European Workshop On Structural Health Monitoring (EWSHM 2016), Bilbao, 2016.

[79] K. Worden and G. Manson, "The application of machine learning to structural health monitoring," Philosophical Transactions of the royal society a, vol. 365, no. 1851, 12 December 2006.

[80] A. Oord, S. Dieleman and B. Schrauwen, "Deep content-based music recommendations," in Thirty-fourth Conference on Neural Information Processing Systems.

[81] R. Collobert and J. Weston, "A unified architecture for natural language processing: deep neural networks with multitask learning," in ICML '08: Proceedings of the 25th international conference on Machine learning, Ney York City, 2008.

[82] O. Abdeljaber, O. Avci, M. S. Kiranyaz, B. Boashash, H. Sodano and D. J. Inman, "1-D CNNs for structural damage detection: Verification on a structural health monitoring benchmark data," Neurocomputing, vol. 275, no. 31, pp. 13081317, 2018.

[83] X. Li and X. Wu, "Constructing Long Short-Term Memory based Deep Recurrent Neural Networks for Large Vocabulary Speech Recognition," in International Conference on Acoustics, Speech, and Signal Processing, 2015.

[84] H. Sak, A. Senior, K. Rao, O. İrsoy, A. Graves, F. Beaufays and J. Schalkwyk, "Learning acoustic frame labeling for speech recognition with recurrent neural networks," 2015 IEEE International Conference on Acoustics," in Speech and Signal Processing (ICASSP), Brisbane, 2015.

[85] S. Srihari, "Long-Term.ppt," [Online]. Available: https://cedar.buffalo.edu/ srihari/CSE676/10.7\%20Long-Term.pdf. [Accessed June 2020]. 
[86] S. Hochreiter and J. Schmidhuber, "Long short-term memory," Neural Computation, vol. 9, no. 8, pp. 1735-1780, 1997.

[87] H. Luo, M. Huang and Z. Zhou, "Integration of Multi-Gaussian fitting and LSTM neural networks for health monitoring of an automotive suspension component," Journal of Sound and Vibration, vol. 428, no. 18, pp. 87-103, 2018.

[88] E. Marchi, F. Vesperini, F. Eyben, S. Squartini and B. Schuller, "A novel approach for automatic acoustic novelty detection using a denoising autoencoder with bidirectional LSTM neural networks," in 2015 IEEE International Conference on Acoustics, Speech and Signal Processing (ICASSP), Brisbane, 2015.

[89] H. Sak, A. Senior and F. Beaufays, "Long Short-Term Memory Recurrent Neural Network Architectures," Google, 2014.

[90] L. Li, G. Liu, L. Zhang and Q. Li, "Deep learning-based sensor fault detection using S-Long Short Term Memory Networks," Structural Monitoring and Maintenance, vol. 5, no. 1, p. 51, 2018.

[91] Y. Cheng, H. Zhu, J. Wu and X. Shao, "Machine health monitoring using adaptive kernel spectral clustering and deep long short-term memory recurrent neural networks," IEEE Transactions on Industrial Informatics, vol. 15, no. 2, pp. 987-997, 2018.

[92] J. Brownlee, "How to Reshape Input Data for Long Short-Term Memory Networks in Keras," Machine Learning Mastery, 31 August 2017. [Online]. Available: https://machinelearningmastery.com/reshape-input-data-long-shortterm-memory-networks-keras/. [Accessed 10 April 2020].

[93] L. Brent, "LSTM Autoencoder for Anomaly Detection," Medium, 25 September 2019. [Online]. Available: https://towardsdatascience.com/lstm-autoencoder-foranomaly-detection-e1f4f2ee7ccf. [Accessed April 2020].

[94] R. Kienzler, "Using Keras and TensorFlow for anomaly detection," IBM, 2 March 2018. [Online]. Available: https://developer.ibm.com/technologies/iot/tutorials/iot-deep-learning-anomalydetection-5/. [Accessed April 2020]. 
[95] NASA, "Prognostics Center for Excellence - Data Repository," NASA, [Online]. Available: https://ti.arc.nasa.gov/tech/dash/groups/pcoe/prognostic-datarepository/. [Accessed February 2020].

[96] I. Goodfellow, Y. Bengio and A. Courville, "Autoencoders," in Deep Learning, Cambridge, MIT Press, 2016.

[97] M. Stewart, "Generating Images with Autoencoders," Towards Data Science, 14 April 2019. [Online]. Available: https://towardsdatascience.com/generatingimages-with-autoencoders-77fd3a8dd368. [Accessed May 2020].

[98] D. P. Kingma and J. L. Ba, "ADAM: A METHOD FOR STOCHASTIC OPTIMIZATION," in ICLR 2015, 2015.

[99] K. Bennett and E. Parrado-Hernandez, "The Interplay of Optimization and Machine Learning Research," Journal of Machine Learning Research, pp. 12651281, 2006.

[100] J. Heaton, "The Number of Hidden Layers," Heaton Research, 1 June 2017. [Online]. Available: https://www.heatonresearch.com/2017/06/01/hiddenlayers.html\#: :text=The\%20number\%20of\%20hidden\%20neurons, size\%20of\%2 0the\%20input\%20layer.. [Accessed March 2020].

[101] D. Li, A. Cong and S. Guo, "Sewer damage detection from imbalanced CCTV inspection data using deep convolutional neural networks with hierarchical classification," Automation in Construction, vol. 101, pp. 199-208, 2019.

[102] S. Hochreiter and J. Schmidhuber, "LONG SHORT-TERM MEMORY," Neural Computation, vol. 9, no. 8, pp. 1735-1780, 1997.

[103] A. Aravelli, D. McDaniel, M. Thompson, K. Imrich and B. Wiersma, "ErosionCorrosion Detection in Carbon Steel Pipe Loops using SRNLs Thickness and Mass Loss Measurement Coupons," in Proceedings of Waste Management Symposia, Phoenix, 2020. 